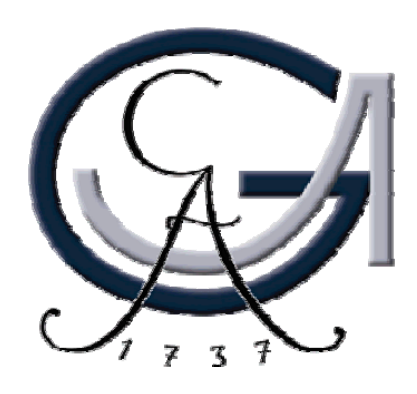

\title{
Actin turnover regulates mechanical properties of oligodendrocytes and myelin formation
}

\author{
Dissertation \\ for the award of the degree \\ "Doctor of Philosophy" (Ph.D.) \\ in the doctoral program "Molecular Biology of Cells" \\ at the Georg-August-Universität Göttingen
}

submitted by

Paula Verónica Sánchez Baeza

from Temuco, Chile

Göttingen 2015 


\section{Members of the thesis committee}

Dr. Iwan A.T. Schaap (Reviewer)

III. Physical Institute

Georg August University Göttingen

Prof. Dr. Mikael Simons (Reviewer)

Max Planck Institute for Experimental Medicine

Department of Neurology, Georg August University Göttingen

Prof Dr. Andreas Janshoff

Institute for Physical Chemistry

Georg August University Göttingen

\section{Additional members of the examination board}

Prof. Dr. Klaus-Armin Nave

Department of Neurogenetics

Max Planck Institute for Experimental Medicine

Prof. Dr. Tiago Fleming Outeiro

Department of Neurodegeneration and Restorative Research

University Medical Center Göttingen

Prof. Dr. Sarah Köster

Institute for X-Ray Physics

Georg August University Göttingen

Date of the oral examination: $8^{\text {th }}$ July, 2015 


\section{Affidavit}

I hereby declare that this $\mathrm{PhD}$ thesis entitled "Actin turnover regulates mechanical properties of oligodendrocytes and myelin formation" has been written independently with no other sources that quoted.

Paula Veronica Sanchez Baeza

May, 2015

Göttingen, Germany 



\section{Contents}

Affidavit.................................................................................................................................... iii

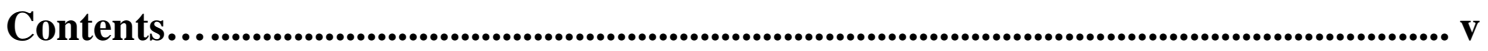

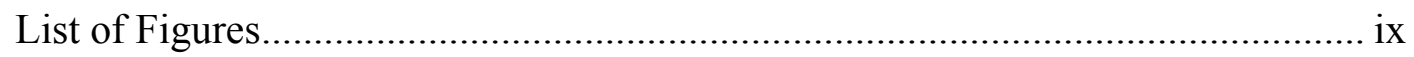

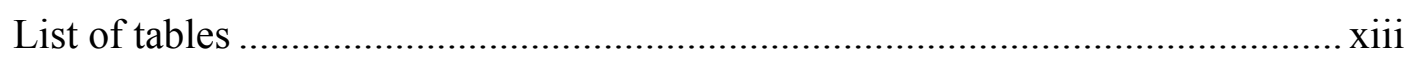

Abbreviations....................................................................................................................... XV

Acknowledgments................................................................................................................... xix

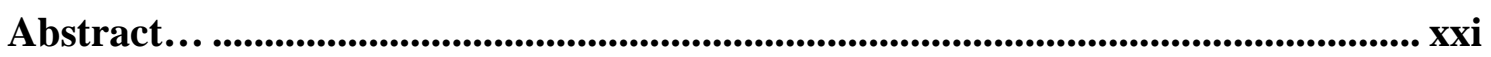

1 Introduction... ............................................................................................................. 1

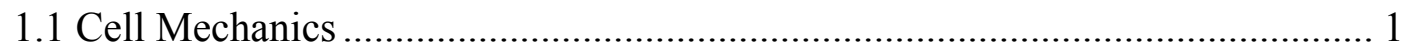

1.1.1 Cellular processes related to cell mechanics ........................................ 2

1.1.2 Describing mechanical properties of cells ............................................. 4

1.1.3 Techniques used to measure mechanical properties.............................. 8

1.2 Myelin sheath formation............................................................................... 18

1.2.1 Myelin in the Central Nervous System.............................................. 18

1.2.2 Myelin development in the CNS ....................................................... 21

1.2.3 Model of myelination in the Central Nervous System (CNS) ............ 24

1.2.4 Actin filaments and force generation................................................. 26

1.3 Aims of the Project................................................................................. 30

2 Materials and Methods........................................................................................... 31

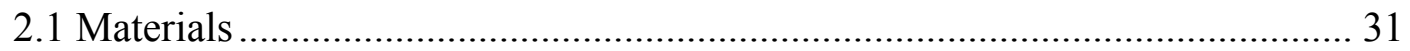




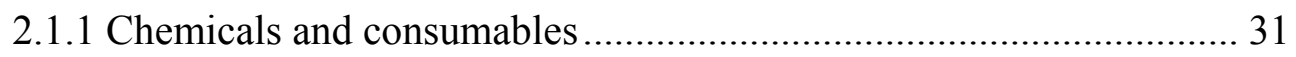

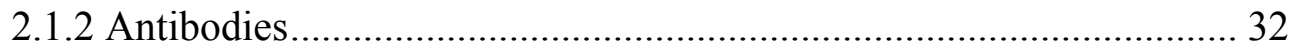

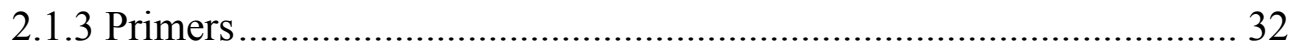

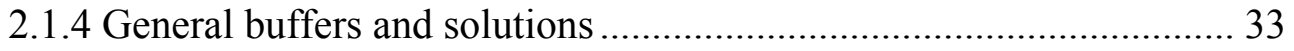

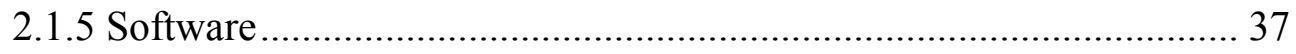

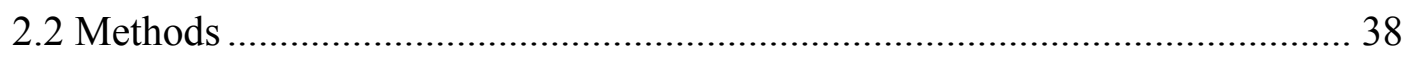

2.2.1 Atomic Force Microscopy ........................................................ 38

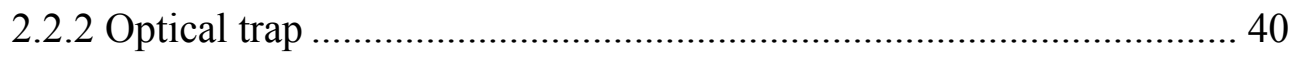

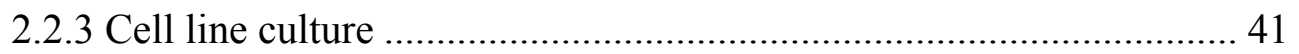

2.2.4 Primary Oligodendrocyte culture .................................................. 41

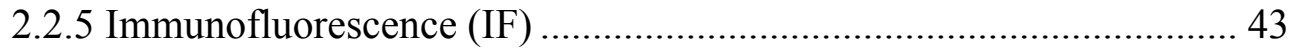

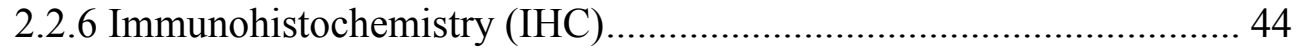

2.2.7 Interference Reflection Microscopy (IRM) .................................... 45

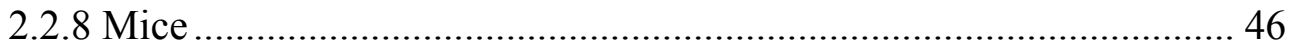

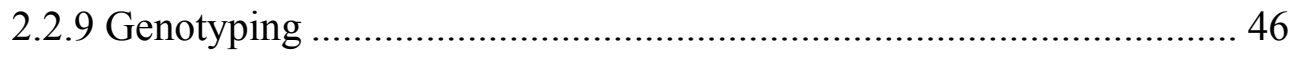

2.2.10 Agarose gel electrophoresis .................................................. 48

2.2.11 Image processing and statistical analysis .................................. 48

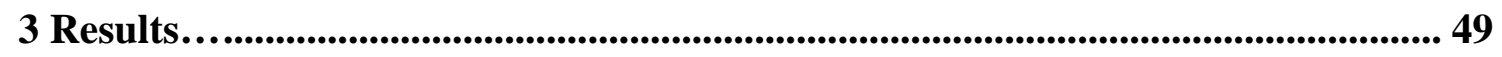

3.1 Cell visco-elasticity measured with AFM and optical trapping at sub-

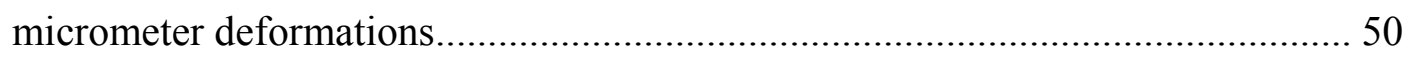

3.1.1 Response of cells to mechanical deformations ............................... 50

3.1.2 The cell's response is elastic at small deformations and viscous at

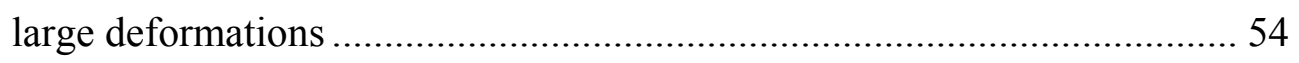

3.1.3 The elastic response of cells at small deformations is anisotropic .... 56

3.1.4 The actin cortex is the major contributor to cell elasticity at small

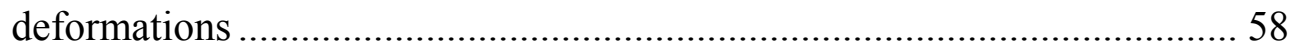

3.2 Actin filament turnover drives leading edge growth during myelin sheath formation in the Central Nervous System 
3.2.1 The leading edge of oligodendrocytes shows an adhesion independent model of protrusion 59

3.2.2 Membrane motility ceases and adhesive contacts are formed after actin depolymerization .... 64

3.2.3 Oligodendrocytes build up a membrane reservoir during differentiation

3.2.4 F-actin depolymerization decreases surface tension

3.2.5 The actin depolymerizing factors ADF and Cofilin1 are responsible for low $\mathrm{F}$ - to $\mathrm{G}$ - actin ratios

3.2.6 ADF and Cofilin1 are required for myelin growth but not for its maintenance

4 Discussion. 81

4.1 Validation of a vertical optical trap for mechanical measurements 81

4.2 The response of cells to small deformations is elastic but anisotropic 84

4.3 The elastic response of cells is determined by the actin cortex. 86

4.4 The response of cells to large deformations has a major viscous component.. 86

4.5 Actin distribution during myelination 88

4.6 Shift between two migratory mechanisms allows wrapping of the leading edge 89

4.7 Membrane spreading as a wetting transition.... 91

4.8 The factors ADF/Cofilin1 are necessary for actin depolymerization and myelin growth. 93

4.9 Proposed model for the role of actin in myelin formation 94

5 References.. 97

Curriculum Vitae. 107

List of publications. 109 


\section{List of Figures}

\section{Introduction}

Figure 1.1: Elasticity values of biological samples ...................................................... 5

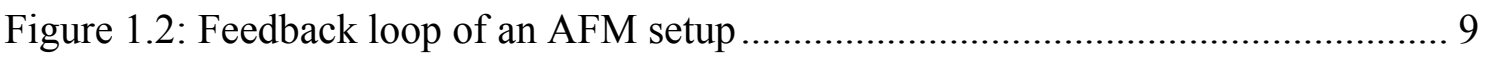

Figure 1.3: Forces acting on the particle during optical trapping............................... 14

Figure 1.4: Photograph of our vertical optical trap ............................................... 16

Figure 1.5: Compacted and non-compacted myelin .................................................. 20

Figure 1.6: Factors influencing oligodendrocyte differentiation and myelination......... 23

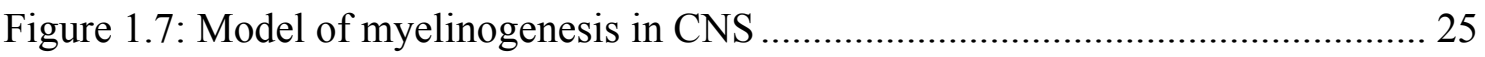

\section{Results}

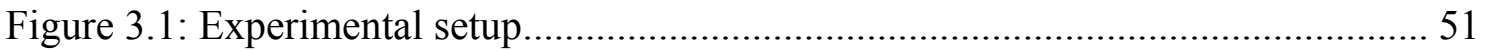

Figure 3.2: The response of cells at higher forces shows an increased hysteresis ......... 52

Figure 3.3: Higher deformations display a loss of energy between indentation and retraction.

Figure 3.4: The apparent Young's modulus of the cells suggests the presence of a viscous component at higher deformations 55

Figure 3.5: The response of the cells is viscous at high deformations and follows a power law 56

Figure 3.6: Directionality of the elastic response is anisotropic 
Figure 3.7: The actin cortex is the major contributor to cell elasticity at small deformations 58

Figure 3.8: F-actin is redistributed during oligodendrocyte differentiation.... 60

Figure 3.9: The leading edge of oligodendrocyte precursors is more adhesive than the leading edge of sheet forming oligodendrocyte

Figure 3.10: Disruption of the cell-substrate interactions collapses the leading edge of precursor but not sheet forming oligodendrocytes

Figure 3.11: Vinculin is only present it early stage OPCs

Figure 3.12: Membrane motility decreases after actin depolymerization 64

Figure 3.13: Adhesive areas increase upon actin depolymerization 65

Figure 3.14: F-actin depolymerization triggers membrane spreading. 66

Figure 3.15: F-actin stabilization decreases motility but does not trigger membrane spreading

Figure 3.16: Oligodendrocytes build up a large membrane reservoir during differentiation 67

Figure 3.17: Depolymerization of F-actin reduces membrane tension 70

Figure 3.18: ADF and Cofilin1 are upregulated during differentiation 72

Figure 3.19: Generation and genotyping of ADF-Cofilin1 KO (AC DKO) mice. 73

Figure 3.20: F-actin localization at the leading edge and F- to G-actin ratios are significantly higher in ADF-Cofilin1 DKO mice 74

Figure 3.21: Increase of F-actin levels located at the leading edge of ADF Cofilin1 DKO mice. 75

Figure 3.22: $\mathrm{F} / \mathrm{G}$ actin ratio of ADF-Cofilin1 DKO animals is higher as compared to controls 76

Figure 3.23: ADF/Cofilin1 are required for myelin biogenesis in mice 77 
Figure 3.24: The number of oligodendroglial cells between control and ADF/Cofilin1 DKO mice is comparable 78

Figure 3.25: Inducible ADF-Cofilin DKO mice show no difference in myelin compared with control animals

\section{Discussion}

Figure 4.1: Leading edge in oligodendrocytes at different stages of differentiation...... 89

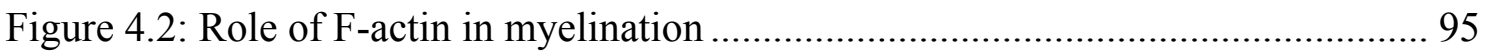




\section{List of tables}

\section{Materials and Methods}

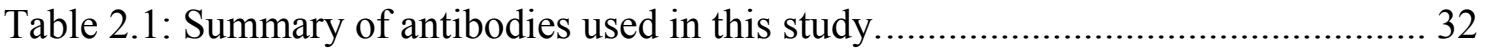

Table 2.2: Summary of primers used for genotyping .................................................. 33

Table 2.3: Summary of software used in this study. ................................................. 37

Table 2.4: PCR programs used for genotyping. ..................................................... 47 


\section{Abbreviations}

$3 \mathrm{D}$

three dimensional

A.U. arbitrary units

AC DKO ADF/Cofilin1 double knockout

ADF actin depolymerizing factor

ADP adenosine diphosphate

AFM atomic force microscopy

ANOVA analysis of variance

ARP2/3 actin-related-protein complex

ATP adenosine triphosphate

BME basal medium eagle

Caspr2 contactin-associated protein

CCD charge-coupled device

CNP 2',3',-cyclic-nucleotide 3'-phosphodiesterase

CNS central nervous system

Ctrl control

div days in vitro

DMEM Dulbecco's modified Eagle's medium

DNA deoxyribonucleic acid

ECM extracellular matrix

EM electron microscopy 


\begin{tabular}{|c|c|}
\hline F-actin & filamentous actin \\
\hline FBS & fetal bovine serum \\
\hline fl & floxed \\
\hline G-actin & globular actin \\
\hline $\mathrm{H}$ & hour \\
\hline HS & horse serum \\
\hline IF & immunofluorescence \\
\hline $\operatorname{IgG}$ & immunoglobulin $\mathrm{G}$ \\
\hline $\operatorname{IgM}$ & immunoglobulin $\mathrm{M}$ \\
\hline IHC & immunohistochemistry \\
\hline IRM & interference reflection microscopy \\
\hline $\mathrm{k}$ & spring constant \\
\hline KO & knockout \\
\hline LED & light-emitting diode \\
\hline MAPK & mitogen-activated protein kinase \\
\hline MBP & myelin basic protein \\
\hline MOG & myelin oligodendrocyte glycoprotein \\
\hline MS & multiple sclerosis \\
\hline n.s. & non significant \\
\hline $\mathrm{N} / \mathrm{m}$ & newton per meter \\
\hline NA & numerical aperture \\
\hline NCAM & neural cell adhesion molecule \\
\hline Necl & nectin-like molecule \\
\hline NG2 & neural/glial antigen 2 \\
\hline OLG & oligodendrocyte \\
\hline
\end{tabular}


xvii

OPC oligodendrocyte precursor cell

$\mathrm{P}(\mathrm{n}) \quad$ post natal day

PB phosphate buffer

PBS sodium phosphate buffer

PCR polymerase chain reaction

PDGF platelet-derived growth factor

PDGFR $\alpha \quad$ platelet-derived growth factor receptor alpha

PFA paraformaldehyde

PI3K phosphatidylinositol-4,5-biphosphate 3-kinase

PLL poly-1-lysine

PLP proteolipid protein

PNS peripheral nervous system

GTPase guanosine triphosphate hydrolase

SD standard deviation

SEM standard error of the mean

UV ultraviolet

WASP Wiskott-Aldrich syndrome protein

WT wildtype 


\section{Acknowledgments}

I will like to thank my supervisor Dr. Iwan Schaap for believing in me from the very beginning when I had no clue what biophysics meant. I have learned and grown so much by being part of the group. Thanks for always being available for discussions and guidance not only on a strictly professional manner. I will also like to thank my supervisor during the last one and a half years of my PhD, Prof. Dr. Mikael Simons for reminding me about how much I loved "bench work", for trusting in me and pushing my limits into almost insanity for a very good cause. I'm also very grateful to the third member of my thesis committee Prof. Dr. Andreas Janshoff for fruitful discussion and collaborations.

Thanks to all my beloved colleagues and friends from the biophysics group at the Third Institute of Physics, for making working and (specially) non-working hours so much fun!, to our T.A.s and secretaries, particularly Kerstin for being always so friendly and kind and Nicole for all the help with absolutely every issue I could come up with. To Dr. Florian Rehfeldt for all the support during my time at the DPI and his very funny but twisted sense of humor. I will also like to thank in particular to Mijta for being the coolest office mate and friend (together with Kalina, of course), Alok for being so candid and caring, "Papa" Chris for all the meaningful advices about life, my two "rockfellas": Flo for making us "go with the flow" and André for being so special and believing blindly everything I said. To my dear friend Christian Blau because even though you are not part of the group you were always willing to "crush" our gatherings. 
Honorable mention for my two best friends and family, Minu and Miquel for so many wonderful moments we have shared together and always finding time for each other. I will carry all of you deeply in my heart.

Thanks to my bestie "B" for all the extremely useful comments on the first draft of this thesis and to my friend Kasia for a very efficient proofreading.

To my lovely colleagues and friends from the AG Simons, for welcoming me in the group with open hearts as soon as I joined and making the time spent in and out of the lab so amazing. Thanks to Giselheid for the outstanding primary cultures and specially for patiently helping me to improve my German unintentionally. To my lab "bros": Ludo (best dancing partner ever) and David for all those late, unimaginable - and irreproducible - conversations.

To by beloved "latin friends": Chepe, Melis, Nana, Pedro, Ritis and Cesar for bringing all that joy and energy wherever you are. To my flat mates, former and present, for all the time spent together and how much you have taught me about life, tolerance and sharing. Special thanks to Katrin for always being there during my good and bad times and for caring so much for all of us. Thanks to Carolina Otero for recommending me as a candidate for this $\mathrm{PhD}$ position and our fruitful collaborations.

A mis "viejas locas" en Temuco, especialmente a mi mama Pilar por haber apoyado todas mis decisiones e incitarme siempre a buscar nuevos horizontes. Por todo el apoyo a pesar de estar tan lejos. Sé que no ha sido fácil para ti y te lo agradezco demasiado. A mi familia postiza, los Baezos, por aceptarme y hacerme una más del clan. A mi vijito por ser el mejor soporte que podría haber tenido durante toda mi época de estudiante y hasta el día de hoy. A panlikuapesinla por ser mis primeros amigos en la $U$ y, a pesar de todos los dramas, mantenernos unidos hasta hoy. 


\section{Abstract}

The myelin sheath is a specialized membranous structure that facilitates rapid signal conduction along axonal segments. During central nervous system development, it is formed by oligodendrocytes that extend motile and exploratory processes. Upon axonal contact, these processes transform into flat sheets that spread and wrap around the axons to generate a multilayered stack of membranes. In order to drive the leading edge of the forming sheath in between the growing myelin layers and the interface with the axon, mechanical forces are necessary, however, the underlying mechanisms are not known. Thus, to study how the process of myelin formation occurs, we used an interdisciplinary approach that combines morphological and genetic analysis with nano-mechanical experiments.

In order to perform mechanical measurements on flat oligodendrocytes with minimum damage, we developed a vertical optical trap and compared its performance with atomic force microscopy. We found that indentation experiments carried out with both instruments yield consistent results for the cell elasticity. I used both complementary techniques to measure the response of fibroblasts over a large range of forces and deformations modes.

Experiments on oligodendrocytes pointed to a key role for the actin filament (Factin) network dynamics in myelin growth. At the onset of myelin biogenesis, F-actin is located primarily at the non-adhesive leading edge, which is propelled around the axon driven by forces generated by F-actin polymerization. Behind the leading edge, F-actin disassembly reduces surface tension which allows membrane spreading and 
transforming the processes into large flat sheets that adhere to the substrate. Furthermore, we have identified the actin depolymerizing factor ADF/Cofilin1, as necessary regulator of myelin formation. By controlling the local actin dynamics with repetitive cycles of assembly and disassembly, oligodendrocytes can drive the protrusions forwards and eventually spread. 


\section{\begin{tabular}{l|l} 
Introduction & 1
\end{tabular}}

\section{$1.1 \mid$ Cell Mechanics}

Throughout the years, the functioning of cells has been approached by biochemical, molecular and genetic methods. This has led to a great understanding of the components of the cell and provided insights into the behavior of cells by elucidating processes such as signaling pathways, ligand-receptor or substrate-enzyme interactions and regulation of the cytoskeleton, to only name a few. More recently, mechanical properties of cells are increasingly appreciated as an additional information channel to better understand the structure and behavior of cells. Many of the concepts and ideas about cell mechanics have been around for a long time (Pelling and Horton, 2008) and the last decade has seen a strong increase in studies related to the topic (Guck and Chilvers, 2013). This is, on one side, due to the development of new and improved techniques used to measure cell mechanical properties. On the other side, more connections are being discovered that link the physical and mechanical properties of cells to relevant biological processes (Christopher R. Jacobs, 2012). 


\subsubsection{Cellular processes related to cell mechanics}

A variety of cellular processes on different levels of complexity are directly or indirectly linked to cell mechanics. Our skeletal system is one example illustrating this, where bone tissue and cartilage serve as a mechanical support for our body and muscular system. The state of the skeleton is not a static, but dynamic condition since on one hand deprivation of mechanical stimulation leads to a debilitation of bone tissue and on the other hand, mechanical load activates various signaling pathways leading to bone formation (Huiskes et al., 2000; Jacobs et al., 2010). Similar effects have been found on cartilage, where moderate and cyclic stimuli are beneficial for chondrocytes, while excessive chronic mechanical loading will damage the tissue and generate osteoarthritis (Moyer et al., 2014).

On a cellular level, the regulation of cell shape by adjusting their mechanical properties has important functional implications. This is particularly interesting during mitosis, when rounding up of cells takes place as a consequence of disassembled surface adhesion contacts. The resulting decrease in the adhesive forces keeping the cell adhered to the surface as well as a reorganization of the actin cytoskeleton then generates enough mechanical stress to increase the membrane tension at the equator which is the start of the division of the whole cell (Clark and Paluch, 2011).

Apart from internal mechanisms regulating cell shape, the cell's environment likewise exerts effects on their morphology and differentiation. As an example, plating naïve mesenchymal stem cells with no basal expression of any lineage-specific markers onto matrixes of different stiffness (expressed in $\mathrm{kPa}$ ) influences their specification into cells expressing neurogenic $(0.1-1 \mathrm{kPa})$, myogenic $(11 \mathrm{kPa})$ or osteogenic $(34 \mathrm{kPa})$ markers (Engler et al., 2006). Studies relating cell mechanics and stem cells have been 
so successful that the concept of "mechano-niche" has emerged, defining a specific combination of mechanical properties of the cell, the extracellular matrix and other external cues to control the maintenance of the cell population and later differentiation (Lee et al., 2011). It is hence not surprising that also the development of our nervous system is tightly regulated by cell mechanical processes (Franze, 2013). One example is the distinct mechanical properties of central nervous system (CNS) cell types, with astrocytes being structurally softer than neurons (Lu et al., 2006). Since in mixcultures of cortical neurons and astrocytes plated onto soft substrates neuronal growth is favored (Georges et al., 2006), it raises the idea that in addition to being structural support astrocytes provide a soft mechanical environment highly favorable for neuronal development.

Generally, for a cell to react to a certain mechanical stimulus, signal transduction has to take place. In a first step, the cell is mechanically deformed in response to a stimulus which then, in a second step is translated into an active biochemical pathway by mechanosensors. Mechanosensitive channels e.g. provide the cell with a direct mean to assess the membrane's stretching state by switching between open and closed conformations (Sachs, 2010). Indirect means include for instance, the focal adhesion complexes, which are macromolecular protein assemblies connecting the extracellular matrix $(\mathrm{ECM})$ and the cytoskeleton and have been shown to respond to applied force by assembly or disassembly of its components (Geiger and Bershadsky, 2002; Harburger and Calderwood, 2009).

Taken together, numerous cellular processes are controlled by the interplay between the cell's mechanical properties and external mechanical forces acting on it with the cytoskeleton being a crucial structural component at the center of its regulation. 
4 Introduction

\subsubsection{Describing mechanical properties of cells}

The reaction of a cell to mechanical stimuli (how much it deforms) can be described by parameters that approximate its mechanical properties. In this section I will describe the most relevant ones in the context of this study.

\subsubsection{Elasticity}

If material is elastic it will return to its original shape after a deformation has taken place. At small deformations, it follows Hooke's law which states that the force is proportional to the deformation, where $F$ is the applied force, $\Delta x$ is the deformation and $k$ is the factor of proportionality of the material which describes its spring constant in $\mathrm{N} / \mathrm{m}$.

$$
F=-k \Delta x
$$

Analogous to Hooke's law the elasticity of a 3 dimensional solid can be quantified by the Young's modulus $(E) . E$ is the factor of proportionality (in $\mathrm{N} / \mathrm{m}^{2}$ ) between the tensile stress $(\sigma$; the force $F$ per area $A)$, and the tensile strain $(\varepsilon$; the relative change in length $\left.\Delta l / l_{0}\right)$.

$$
E=\frac{\sigma}{\varepsilon}=\frac{F / A}{\Delta l / l_{0}}
$$

When the force is not applied uniaxial but perpendicular one refers to the shear modulus $\left(G^{\prime}\right)$. For all elasticity parameters a higher value means that the object is harder to deform. The cell does not behave like an ideal elastic material which is a consequence of its heterogeneous composition. Nevertheless, in many publications the Young's modulus is used to quantify the stiffness of the cell. Although not entirely 
correct it allows one to compare different measurement at least when the experimental procedures are comparable (Atanackovic and Guran, 2000; Sadd, 2005).

Different biological samples have reported Young's moduli that cover a wide range of elastic values from hundreds of $\mathrm{Pa}$ to $\mathrm{GPa}$ which correlates with their function in the organism, as shown in Figure 1.1.

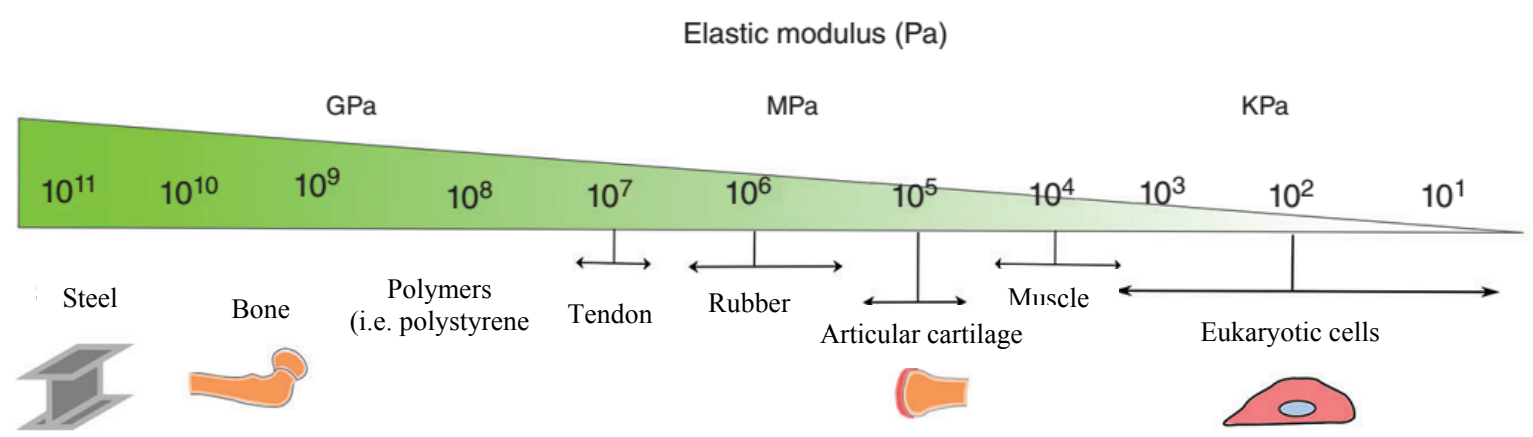

Figure 1.1: Elasticity values of biological samples. Scheme showing elasticity of biological materials compared to steel and rubber. Figure adapted from Moeendarbary, E., and Harris, A.R. (2014).

\subsubsection{Visco-elasticity and rheology}

If a material is viscous it will not return to its original shape after a deformation has taken place. Viscosity $(\mu)$ is considered as a property of liquids describing the rate at which a fluid flows under a defined load or more specifically, its resistance to stress. It is calculated by the ratio of stress to the rate of strain or flow rate $(\gamma)$ (Maxwell, 1866).

$$
\left.\sigma=\mu^{*} \gamma \quad \text { (Eq. } 3\right)
$$

Cells do neither behave like an ideal elastic material nor like an ideal viscous material. Instead they exhibit both elastic and viscous properties, meaning that they often return only partially to their original shape after a deformation. This combined behavior results from the heterogeneous composition of cells from stiff cytoskeletal filaments and the more fluid such as cytoplasm. Various models exist to quantify the visco-elasticity at which elastic and viscous elements are placed in series or in parallel 
6 | Introduction

(Maxwell and Kelvin-Voigt materials, respectively). Since none of these models describe accurately the mechanical behavior of the cell they were not used for this thesis work and will not be described in more detail (Viswanath et al., 2007).

As mentioned before, the response of cells to mechanical deformation includes both elastic and viscous components. This is one of the reasons for the high variation in the reported values for the cell's Young's modulus. When a visco-elastic cell is deformed quickly it will appear stiffer than when the same cell is deformed slowly; the viscous components will result in a deformation rate-dependent response. Although an exact modeling of this response remains difficult, a consistent behavior has been observed when comparing different measurements that were performed at a range of deformation rates. Basically, in such measurements the cell is deformed with a probe that is oscillated at a range of frequencies, between single and a few hundred Hz. Such rheology experiments measure the frequency dependent response of materials and show typically a stiffening of the cell at higher frequencies. The thus obtained visco-elastic modulus $\left(E^{*}\right)$ consists of the elastic modulus $\left(E^{\prime}\right)$ and the viscous component $\left(E^{\prime \prime}\right)$. The frequency dependence of the elastic modulus of a material is characteristic to its structure. As is turns out even when the absolute Young's modulus is not the same in the experiments on cells from different research groups there is often a remarkable agreement between their qualitative frequency dependency (Hoffman and Crocker, 2009). In most cases the frequency-dependent stiffness of cells has been found to follow a power law:

$$
E^{*}=b^{*} \omega^{\alpha}
$$

Where $b$ is a prefactor that sets the absolute stiffness, $\omega$ the frequency ( $\sim$ deformation rate) and $a$ the power, which has a value between 0.1 and 0.3 depending on which part of the cell is probed (Hoffman et al., 2006). While techniques measuring 
the "outside" of cells e.g. magnetic twisting cytometry, atomic force mictoscopy, external laser tracking, micropipette aspiration or uniaxial rheometry will yield exponents on the range of $0.13-0.17$, techniques measuring the "inside" of the cell such as internal laser tracking and two-point microrheology will result in a higher exponent ranging from 0.24-0.29 (Hoffman et al., 2006). This can be explained by the fact that the "outside" techniques are measuring the response at the actin cortex region, while the "inside" techniques are testing the deep intracellular space around the nucleus (Hoffman and Crocker, 2009; Hoffman et al., 2006).

\subsubsection{Surface tension}

Eukaryotic cells are enclosed by a lipid bilayer that is supported by $\sim 100 \mathrm{~nm}$ thick actomyosin cortex. Because this layer has a relative large influence on the mechanical properties of the cell some measurements can be actually very well described by the surface tension or membrane tension. This tension depends on the inplane tensile force within the outer layer of the cell divided by the thickness of this layer. For cells the tension is the sum of the in-plane tension of the lipid bilayer and the cortical tension which is largely dependent on the actomyosin cortex (Christopher R. Jacobs, 2012; Clark and Paluch, 2011). A classical experiment to estimate the surface tension values of a cell is the tether extraction measurement (Hochmuth et al., 1996). First, a probe is attached to the cell and then pulled away. When the attachment between the cell and probe is firm enough small tubes of membrane bilayer called tethers are extracted, which are detached from the actomyosin cortex. The measured static tether force ( $F_{0}$, force necessary to keep the pulled tether at a constant length), contains the contribution of the bending stiffness of the bilayer $(B)$, the in-plane membrane tension, and membrane-cytoskeleton adhesion (Dai and Sheetz, 1997; Dai and Sheetz, 1999; Dai 
8 Introduction

et al., 1998; Diz-Munoz et al., 2013; Sheetz, 2001; Sheetz and Dai, 1996). The last two terms cannot be separated, since the in-plane tension is generated by the cytoskeleton adhesion and therefore are combined into the so-called membrane tension $\left(T_{m}\right)$.

$$
F_{0}=2 \pi \sqrt{2 B T_{m}}
$$

\subsection{3 $\mid$ Techniques used to measure mechanical properties}

There are many techniques to experimentally determine the mechanical properties of cells. In this section, I will provide insight into the techniques applied during this study, namely atomic force microscopy (AFM) and optical tweezers.

\subsubsection{Atomic Force Microscopy}

AFM belongs to the family of scanning probe microscopes and was first described by Binnig, Quate and Gerber in 1986 (Binnig et al., 1986). It comprises a tip (probe) mounted at the end of a flexible cantilever that can be controlled using piezoelectric actuators. Since the cantilever is flexible, any contact of the tip with the surface or sample will apply force onto the cantilever end and result in bending. The deflection is measured by focusing a laser on the back of the cantilever and projecting its reflection onto a quadrant photodiode (Allison et al., 2010; Kapanidis and Strick, 2009; Last et al., 2010). The z-movement of the cantilever with respect to the surface is controlled by a feedback loop that allows controlling the bending of the cantilever which can be either kept constant via the force applied to the tip or kept oscillating at a constant amplitude (Figure 1.2). Briefly, when the cantilever bends, the angle of reflection changes and therefore the laser spot will move away from the center of the 
quadrant detector. The detector signal is processed by the controller and compared with the set-point (the desired cantilever bending). The controller will send a signal to the piezo actuator to move the cantilever down until the set point is reached after which the motion is stopped (Eghiaian and Schaap, 2011). This control is so precise that it allows obtaining a sub-nanometer resolution in the vertical axis (Last et al., 2010).

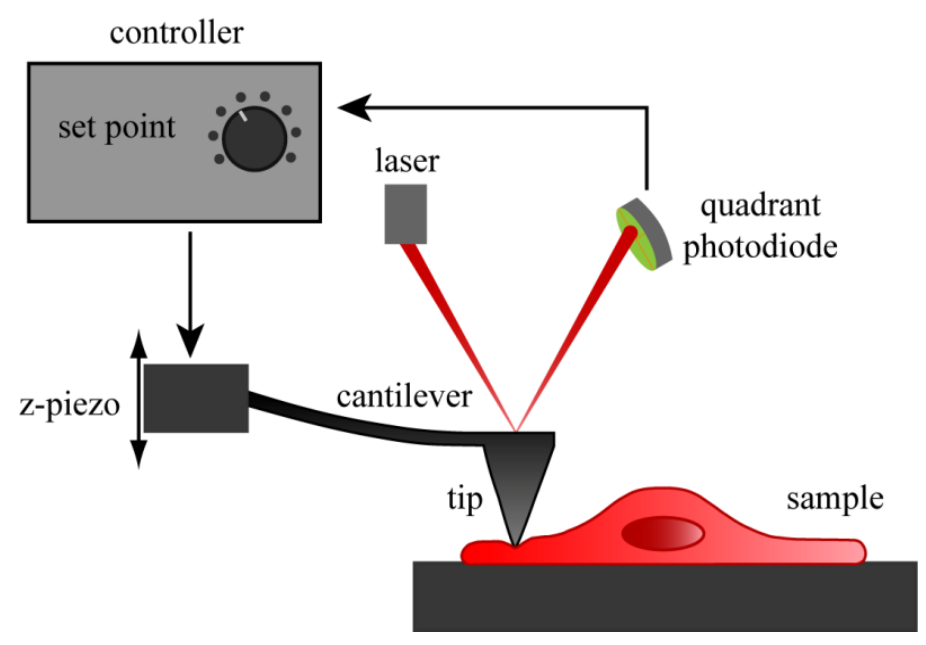

Figure 1.2: Feedback loop of an AFM setup. The external controller receives information from the photodiode and compares it with the set point. If they are unequal the cantilever is moved accordingly.

There are two widely used modes of imaging that can be used depending on the type of sample. In contact mode the cantilever approaches and touches the surface until the set point is reached. From there it starts scanning in $\mathrm{x}$ and $\mathrm{y}$ while keeping the cantilever bending constant. When the tip touches the sample, which is higher than the surrounding surface, the bending will increase and the feedback loop will retract the cantilever until the set point is restored. From the $\mathrm{x}, \mathrm{y}, \mathrm{z}$ signals that are sent to the piezo actuators it is possible to reconstruct the topography of the sample. The disadvantage of this mode is that the lateral forces exerted on the sample can reach high levels. When the specimen is not well attached to the surface or is easily deformable this can result in uncontrolled deformations or even its displacement (Allison et al., 2010; Eghiaian and Schaap, 2011). 
It is possible to reduce the lateral forces by working in tapping mode. In this mode the cantilever is oscillated at its resonance frequency (Eghiaian and Schaap, 2011). In this case, the set point is defined as the desired amplitude of oscillation. As the oscillating cantilever approaches to the surface, the amplitude decreases due to tipsurface interactions, the feedback look will adjusts its height accordingly to keep the amplitude constant (Allison et al., 2010; Eghiaian and Schaap, 2011; Martin et al., 1987; Putman et al., 1994).

AFM can also be used to perform force spectroscopy measurements by using the tip to deform the sample and measuring the forces that result from this deformation. In addition, it is possible to extract cell adhesion energies and tether forces from the force curves. First, the tip is brought down to contact the cell for multiple seconds to ensure adhesion between the tip and cell. Then the cantilever is retracted again and the cell is effectively stretched between the surface and the tip. During retraction the cell will at some point detach from the tip, from which the adhesion energies can be extracted. In some cases a membrane tether will be extracted from the cell from which the membrane tension can be extracted. To investigate the spatial distribution of the mechanical properties an automated array of multiple force curve can be performed. Such force maps can for example reveal heterogeneities at different positions of the sample (Allison et al., 2010; Eghiaian and Schaap, 2011; Last et al., 2010).

In order to obtain reliable values for the cell stiffness (Section 1.1.2) it is necessary to calibrate the spring constant of the cantilever properly. During an indentation measurement, the cell $\left(k_{\text {cell }}\right)$ and cantilever $\left(k_{\text {cantilever }}\right)$ will act as two springs in series $\left(k_{\text {total }}\right)$, and the measured stiffness will be determined by both (Eq. 6). When the cantilever spring constant is know this is simply subtracted from the combined value to obtain the spring constant of the cell (Eq. 7). 


$$
\begin{gathered}
\frac{1}{k_{\text {total }}}=\frac{1}{k_{\text {cell }}}+\frac{1}{k_{\text {cantilever }}} \text { (Eq. 6) } \\
k_{\text {cell }}=\frac{k_{\text {cell }} * k_{\text {cantilever }}}{k_{\text {cantilever }}-k_{\text {cell }}}
\end{gathered}
$$

Normally, the spring constant of the cantilever is provided by the manufacturer and depends mainly on its dimensions and the material. These dimensions, especially the thickness, can vary even within the same lot. Also, since most cantilevers have a reflective coating this will have an effect on the spring constant (Neuman and Nagy, 2008). To calibrate the spring constant two steps of calibration are required:

First, the response of the photo detector needs to be calibrated in $\mathrm{m} / \mathrm{V}$, so that we know exactly how many nanometer the AFM tip is moving (by the bending of the cantilever). The photo detector signal is expressed in volts which will make it necessary to convert it into distance units. To achieve this, the cantilever is pressed against the surface therefore the bending will increase linearly when the cantilever is brought closer to the surface. Since the $\mathrm{z}$ piezo actuator is calibrated by the manufacturer we can simply convert the photodetector signal from $\mathrm{V}$ to $\mathrm{nm}$. Basically, a force versus distance curve is performed on the hard surface. The slope of this curve will then indicate how many volts in the photodiode equal a certain displacement of the z-piezo (Neuman and Nagy, 2008).

Second, now we know the displacement of the tip in $\mathrm{nm}$. To convert this into a force we need to multiply it with the spring constant $(\mathrm{N} / \mathrm{m})$ of the cantilever. The spring constant is obtained by recording the thermal noise of the cantilever fluctuations and use the equipartition theorem to calculate the spring constant. The equipartition theorem states that the average energy present in thermal motion of the (cantilever) spring is given by the product of the Boltzmann constant and the absolute temperature:

$$
k_{\text {cantilever }} *<z^{2}>=K_{B} T
$$


The term $<z^{2}>$ gives the positional variance of the cantilever position, which multiplied with $k_{\text {cantilever }}$, gives the average energy of the thermal cantilever fluctuation. One can measure $\left\langle z^{2}>\right.$ directly from the thermal noise recordings. However, this can lead to an over-estimate of the variance because additional noise sources increase the recorded noise. To overcome this problem, the noise signal is converted via a Fourier transformation into a power spectrum which allows distinguishing the real thermal noise of the cantilever from potential other noise sources. From the power spectrum the fundamental resonance frequency of the cantilever is easily distinguished as a large peak. The variance $\left\langle\mathrm{z}^{2}\right\rangle$ of the cantilever deflection is now given by the surface area under the peak which is obtained by fitting the power spectrum with the function of a simple harmonic oscillator (Hutter and Bechhoefer, 1993). In practice the calibration steps are largely automated through the AFM software, and the whole routine can be performed with a few mouse clicks in less than a minute.

There are various advantages speaking for the application of AFM on biological samples such as cells. One of them is that it can be operated in liquid, allowing measurements at near physiological conditions and enabling the observation of dynamic processes such as conformational changes. Also, samples do not have to be fixed or labeled. Cantilevers and tips can be functionalized with specific molecules in order to measure different interactions such as intermolecular interplay (Hinterdorfer and Dufrêne, 2006). Additionally, special tips can be selected to improve imaging conditions or force measurements. An example of this is using sharp tips to improve the resolution of images and blunt spherical tips to distribute the force over a larger area during indentation experiments to avoid damaging of soft samples like cells (Allison et al., 2010; Muller, 2008; Neuman and Nagy, 2008). 


\subsubsection{Optical trap}

The optical trap setup is also referred to as optical tweezers. It is based on the trapping of dielectric particles using a high power focused laser beam. To limit photodamage that may result from the high laser power $(>100 \mathrm{~mW})$, near infra-red wavelengths are used (800-1100 nm). Additionally, to properly focus the laser high numerical aperture objectives are needed (Neuman and Nagy, 2008).

Trapping a particle with a laser beam relies on a highly inhomogeneous spatial field distribution, which is achieved by focusing the beam with a Gaussian intensity profile such that the intensity is highest in the center and decreases towards the edges (Stevenson et al., 2010). A dielectric particle located close to the focus will experience attractive forces towards the focus based on the principle of conservation of momentum (Ashkin, 1997; Neuman and Nagy, 2008) which states that when two bodies collide, they will exert equal forces on each other. In this case, the photons have a momentum, and its direction will change when they travel through the particle, thereby generating a reaction force opposite to this change in direction. Besides this gradient force (Figure 1.3), there is also a scattering force caused by photons that are absorbed or scattered by the particle. The scattering force exerts a force in the direction of propagation of the light, whereas the gradient force acts in the direction of the intensity gradient towards the focus (Ashkin, 1997; Moffitt et al., 2008). Only when the gradient force overcomes the scattering force, the particle is driven into the focus of the optical trap. Once the particle is in the center of the trap, the net force acting on the particle is zero. From then on, it will stay trapped and act as a spring, following Hooke's law with the exerted force being proportional to its displacement from the center of the trap (Moffitt et al., 2008). 


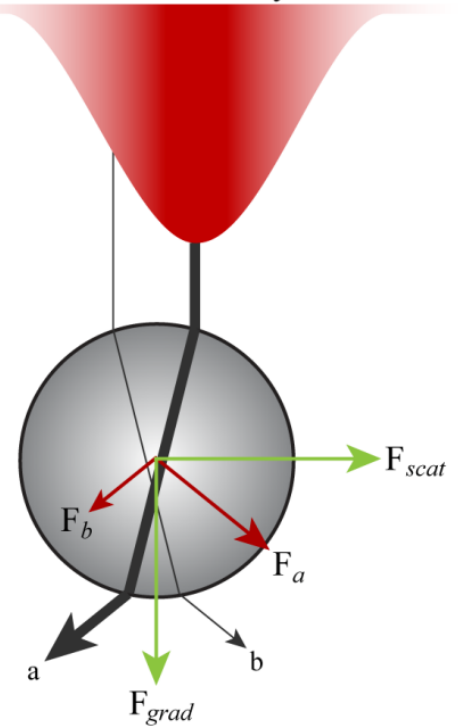

Figure 1.3: Forces acting on the particle during optical trapping. Light is refracted by the particle and it imposes a momentum on it. Gradient forces $\left(F_{\text {grad }}\right)$ will attract the particle perpendicular to the propagation of the beam into the high intensity area and Scatter forces $\left(F_{\text {scat }}\right)$ will push the bead in the direction of the beam.

In order to calibrate and calculate the spring constant of the trapped particle, a similar procedure as for the calibration of the AFM cantilever is followed. The power spectrum of thermal (Brownian) motion of the bead is recorded and the equipartition theorem is again employed to calculate the spring constant. Because the trapped bead behaves like an overdamped spring the power spectrum does not show a pronounced resonance peak. Instead the graph consists of two regimes: At low frequencies the power is constant with a plateau up to the corner frequency. At higher frequencies it decreases linearly with a slope of approximately -2 representing Brownian motion, meaning that the particle is no longer under the confinement of the optical trap. The frequency at which these two curves intersect is the corner frequency, which can be used to calculate the trap stiffness (Bodensiek et al., 2013; Gittes and Schmidt, 1997; Zhang and Liu, 2008). 
In order to calculate the bead displacement from the center of the trap, a quadrant photodiode is placed at a conjugated plane of the back focal plane of the condenser. The interference between unscattered light and the light scattered by the particle will create a particular intensity pattern on the photodiode. Movement of the particle away from the center of the optical trap will result in a shifted light pattern on the photodiode (Bodensiek et al., 2013; Gittes and Schmidt, 1997). Calibration of the sensitivity of the photodiode (in $\mathrm{m} / \mathrm{V}$ ) can be done when the radius of the trapped particle is known. Then the expected Brownian displacements of the bead can be calculated with the Stokes-Einstein equation and the measured displacements can be used to obtain the calibration factor. Similar to the AFM calibration procedure, the calibration steps are largely automated through the optical trapping software, and the whole routine can be performed with a few mouse clicks in less than a minute.

Among the advantages of using an optical trap is the application of very low forces, typically from 1 up to $\sim 100 \mathrm{pN}$. Also, different sized particles can be trapped and consequently used for experimental applications. Furthermore, 3D displacement can be tracked at sub-nanometer accuracy and sub-millisecond time resolution (Neuman and Nagy, 2008).

A detailed description of how our vertical optical trap was built, can be found in (Bodensiek et al., 2013). Briefly, the components for the optical trap were integrated into a commercially available upright microscope. A near-infrared laser (974 nm) was used to minimize photodamage on cells. The optics to guide the trapping laser into the optical path of the microscope was mounted rigidly on top of the microscope to improve resistance against mechanical perturbations. In order to decrease spherical aberrations when trapping particles far away from the coverslip, a water immersion objective was used (Vermeulen et al., 2006). The detection pathway was set up to include vertical (z) 
detection using a quadrant photodiode allowing detection in $\mathrm{x}, \mathrm{y}$ and $\mathrm{z}$ (Dreyer et al., 2004). The advantage of working in the z-direction is that the boundary conditions during deformation experiments are similar to those in AFM. In both cases a probe (the trapped particle or the AFM tip) is brought down to indent the cell in the $\mathrm{z}$ direction, and the results of the measurements can be directly compared. In order to estimate when the trapped particle contacts the cell, a method was developed based on the variability of the thermal bead fluctuations. The principle is that the bead's random fluctuations while being in the trap are much higher than the fluctuations after it is in contact with either a cell or the glass surface since this significantly decreases its freedom of movement. The whole feedback principle of the vertical trap was implemented on a field programmable gate array, a similar approach that is followed by many AFM manufactures and it increased the response time of the feedback loop to the submilisecond time scale (Figure 1.4).
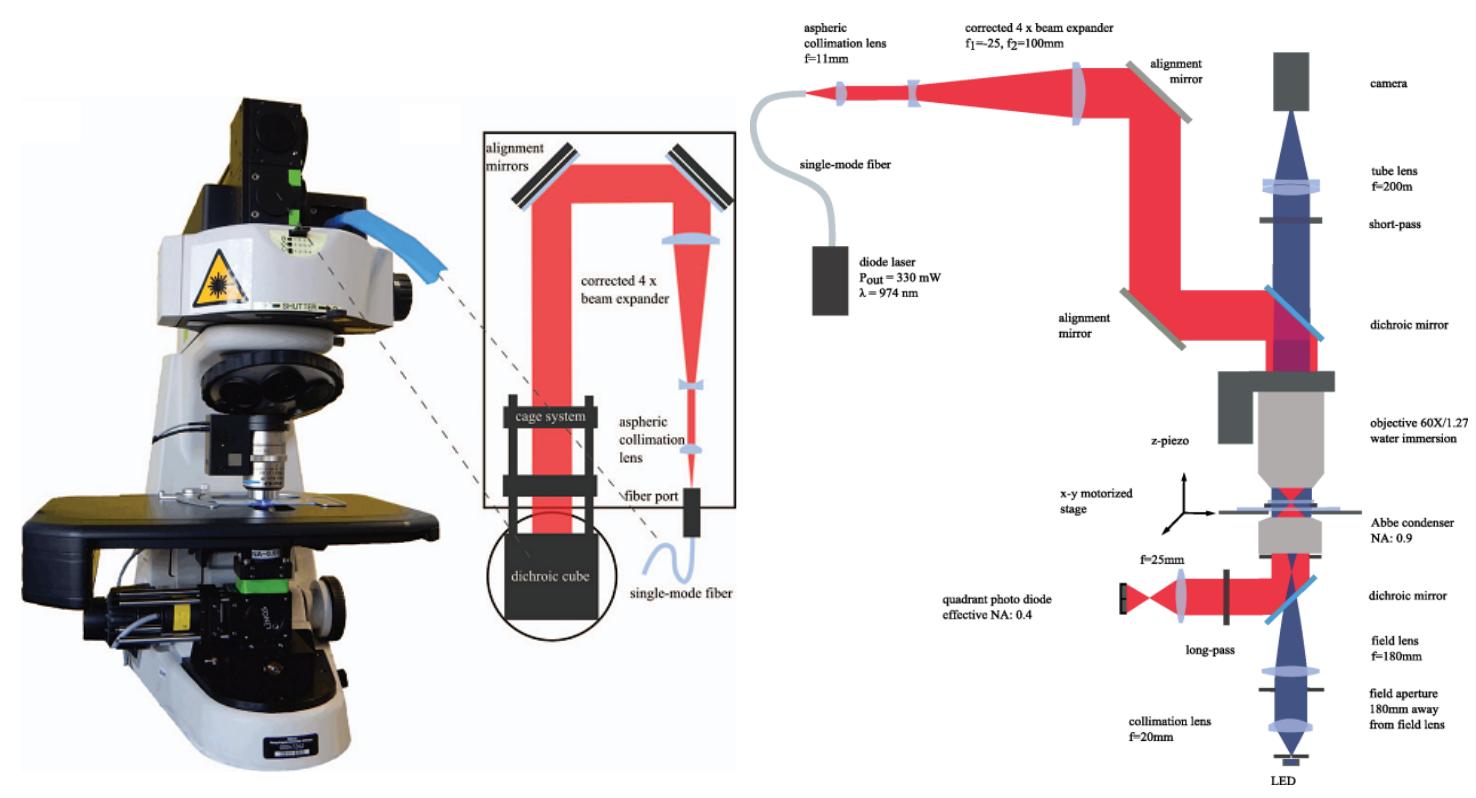

Figure 1.4: Photograph of our vertical optical trap. On top of the microscope a small optical table is mounted that holds most of the optical components that form the optical trap. The light path of the optical table is shown in the middle. The light path of the whole optical trap is shown at the right. The laser light coming from the single mode fiber is collimated and expanded 4 times by a Galileo telescope. The expanded beam is redirected by two mirrors and a dichroic mirror into the optical path of the microscope and the actual trap is formed by the microscope objective lens (Nikon 60x 0.27NA). The vertical position 
of the trap was controlled by a z-piezo that moves the objective up and down. The laser light was collected by the condenser, coupled out of the optical path via a second dichroic mirror and cast onto the photodiode for xyz detection. The illumination source of the microscope consists of a blue LED. Imaging was performed by a standard CCD camera. Image adapted from Bodensiek et al., (2013). Reprint by permission of AIP Publishing LLC, license numbers 3632510635988 and 3637640809569.

The light path of the whole optical trap consists of a laser light coming from the single mode fiber, collimated and expanded 4 times by a Galileo telescope. The expanded beam is redirected by two mirrors and a dichroic mirror into the optical path of the microscope. The actual trap is formed by the microscope objective lens (Nikon 60x $0.27 \mathrm{NA}$ ). The vertical position of the trap was controlled by a z-piezo that moves the objective up and down. The laser light was collected by the condenser, coupled out of the optical path via a second dichroic mirror and cast onto the photodiode for $\mathrm{x}, \mathrm{y}, \mathrm{z}$ detection. The illumination source of the microscope consists of a blue LED. Imaging was performed by a standard CCD camera. 


\subsection{Myelin sheath formation}

\subsubsection{Myelin in the Central Nervous System}

The nervous system is in charge of processing sensory information from all over the body and integrating it into an adequate response. It is divided in two parts, the central nervous system (CNS) comprising the brain and spinal cord, and the peripheral nervous system (PNS) comprising nerves relaying the information from the CNS to the muscles. Due to the immediate relevance of this information for an organism to respond and interact with its environment, substantial parts of this information are transmitted using electrical pulses. This is done by neurons that generate a rapid change in their membrane potential along their longest process, the axon. At the axonal terminal the information is chemically passed on to the next cell. The speed of conduction varies from 0.5 to $10 \mathrm{~m} / \mathrm{s}$ (Neuroscience, 2004), raising a big issue for larger organisms in their capability of responding to environmental stimuli quickly enough. In vertebrates, this problem is addressed by insulating the axon by a thick, multilayered membrane structure called myelin, resulting in increased speed of nerve conduction of up to 150 $\mathrm{m} / \mathrm{s}$ (Neuroscience, 2004). This compacted membrane structure covers segments of the axon of around $150 \mu \mathrm{m}$ in length, locally increasing the resistance and decreasing the capacitance leading to a highly increased signal conduction velocity. In between the myelinated segments lie uncovered segments called nodes of Ranvier, where the electrical signal is regenerated (Hartline and Colman, 2007). Compact myelin is composed of periodic layers of around $12 \mathrm{~nm}$ thick alternating closely condensed 
cytoplasmic myelin membranes and tightly apposed outer membranes (Figure 1.5) (Aggarwal et al., 2011a).

The layered myelin membranes are also called the myelin sheath and largely consists of lipids, accounting for around $70-75 \%$ of its dry weight (Jahn et al., 2009). The most abundant lipids are cholesterol and the glycosphingolipids galactosylceramide and its sulfated form sulfatide (Maier et al., 2008). Other lipids include gangliosides, plasmalogens and phosphoinositides (Schmitt et al., 2014). They not only provide stability of the whole membrane structure by favoring tight packing of molecules but also serve as communicators in axon-glia interactions by locally clustering of proteins or even acting as signaling molecules themselves (Schmitt et al., 2014). The remaining $30 \%$ of myelin dry weight consists of proteins, predominantly the proteolipid protein (PLP) and the myelin basic protein (MBP) (Baumann and Pham-Dinh, 2001). For a long time it had been assumed that the variety of myelin proteins is very low. However, recent improvements in the techniques used to identify myelin proteins have revealed that the aforementioned proteins only constitute $35 \%$ of the newly identified myelin proteins (Jahn et al., 2009). In contrast to the previously known function of myelin proteins of achieving a tight apposition between two membranes, the newly discovered proteins include enzymes, members of the cytoskeleton, protein transporters, trafficking and adhesion-related as well as phospholipid-binding proteins (Jahn et al., 2009). Therefore, the function of proteins in myelin is highly variable.

Myelin is a polarized structure composed of highly compacted areas known as internodes followed by non-compacted regions (Zuchero and Barres, 2011). The internodes are connected to the axonal membrane by the transmembrane proteins Necll and Necl4. Additionally, within the compacted myelin layers, MBP interacts with the cytoplasmic side of the plasma membrane and with neighboring MBP proteins, bringing 
the two bilayers tightly together and acting as a physical barrier excluding other proteins from the compacted areas (Aggarwal et al., 2013; Aggarwal et al., 2011b). Located between areas of compacted myelin and the nodes of Ranvier are another two noncompacted areas of myelin that contact the axon by specific adhesion molecules. First, following the internodal myelin areas are the juxtaparanodes, which interact with the axonal cytoskeleton via adhesion molecules such as Caspr2 and moreover contain $\mathrm{K}^{+}$channels. The juxtaparanodal regions are followed by the paranodes where glia-axon interaction is mediated by the proteins Neurofascin-155 and contactin (Figure 1.5); (Simons and Trajkovic, 2006). These regions are defined by glia-axon interactions and form a lateral diffusion barrier segregating the components of the nodes of Ranvier from the internodal ones in order to allow for a proper transmission of the electric impulse and establishing an area of glia-axon communication that ensures the integrity of the axon (Simons and Trajkovic, 2006).

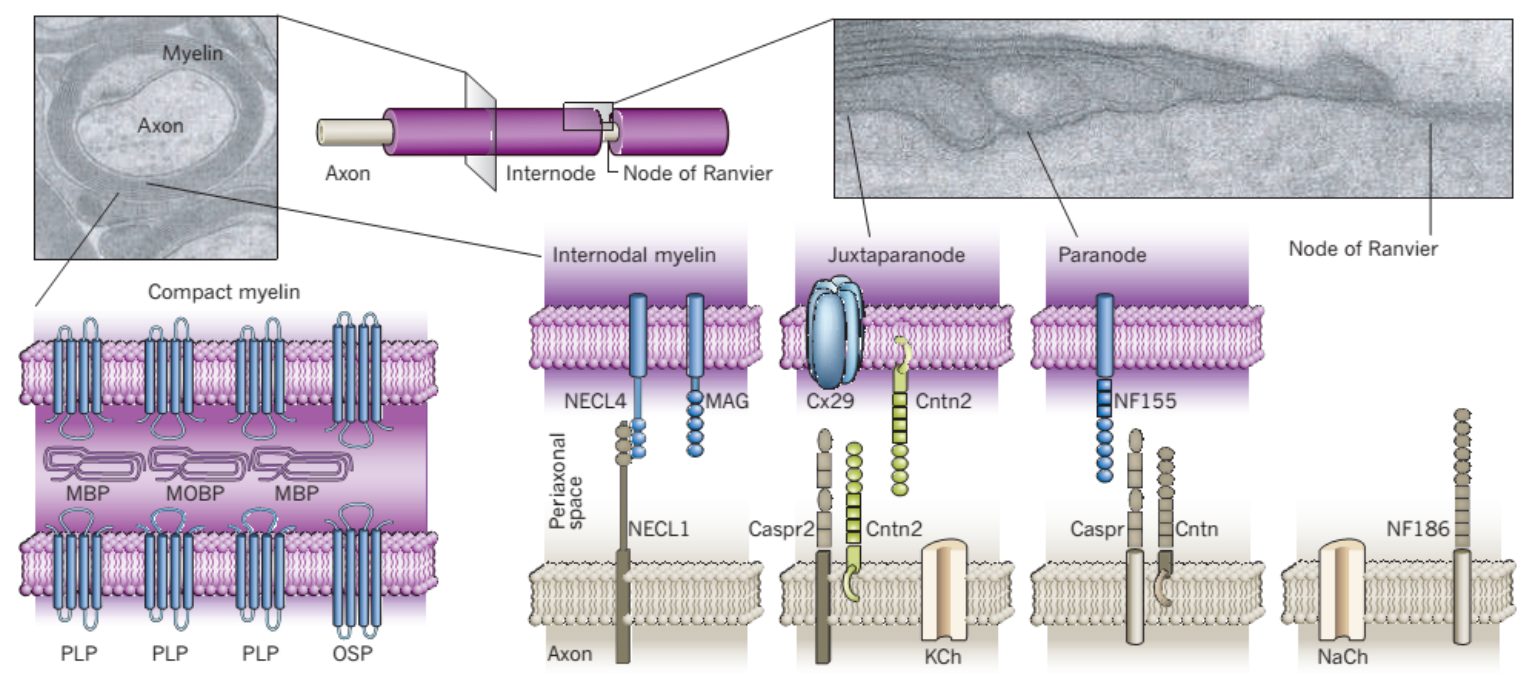

Figure 1.5: Compacted and non-compacted myelin. Electron micrograph on the left shows a crosssection of a myelinated axon. Compacted myelin is tightly brought together by MBP on the cytosolic side and by PLP connecting adjacent layers on the extracellular part of the membrane. Areas devoid of myelin are called nodes of Ranvier and harbors the axonal voltage-gated $\mathrm{Na}^{+}$channels. In between compacted myelin and the nodes of Ranvier there is non-compacted myelin, connected with the axon by different adhesive molecules. Figure adapted from Nave (2010). Reprint by permission of Nature Publishing Group, license number 3632500703833. 
Failure in myelin assembly and maintenance as well as the proper segregation of its components leads to pathology. The most apparent examples for myelin-related pathologies in the CNS are leukodystrophies and multiple sclerosis (MS). In the case of leukodystrophies, genetic mutations are known to affect either assembly or maintenance of the myelin sheath (Nave, 2010), whereas MS is an autoimmune disease in which autoantibodies against epitopes of myelin proteins or the myelin-forming cells are generated (Bradl and Lassmann, 2010; Fitzner and Simons, 2010; Nave, 2010). Both cases lead to dysmyelination and demyelination, respectively, followed by neurodegeneration progressively resulting in motoric disorders and ultimately death. For this reason, many studies are focused on the basic understanding of myelin and myelin diseases in order to develop treatments.

\subsubsection{Myelin development in the CNS}

Myelin sheath in the CNS is produced by a glial cell type called oligodendrocytes. During their tightly regulated differentiation process they undergo dramatic morphological changes. At first, oligodendrocytes extend multiple processes which contact the axon. Then, extensive membrane growth is initiated which eventually forms the myelin sheath around the axon. A single oligodendrocyte is able to produce several myelinated segments on different axons.

Developmentally, oligodendrocyte precursor cells (OPCs) originate from neuroepithelial cells located in a domain of the ventral ventricular zone of the spinal cord under the control of the Notch pathway (Rogister et al., 1999). They express markers such as NG2 and PDGFR $\alpha$ and are able to migrate over long distances to settle 
along the axonal fiber tracts in the areas where they are eventually required to myelinate thereby forming the so-called white matter (Baumann and Pham-Dinh, 2001; Bradl and Lassmann, 2010; Levine et al., 2001; Rogister et al., 1999). Proliferation and migration of OPCs occurs in response to growth factors such as PDGF (Miller, 2002) and ECM proteins in interaction with their glial counterparts located at the OPC cell membrane, e.g. integrin proteins (Milner et al., 1997; Tiwari-Woodruff et al., 2001). Subsequently, they first transform into non-migratory pre-oligodendrocytes expressing sulfatides recognizable by the $\mathrm{O} 4$ antibody (Baumann and Pham-Dinh, 2001) followed by a transformation into pre-myelinating oligodendrocytes that start to express myelin proteins such as PLP/DM20 (Baumann and Pham-Dinh, 2001; Levine et al., 2001). Afterwards, they develop into myelinating oligodendrocytes expressing all myelin proteins (MPB, PLP, CNP, MOG, etc) and processes that did not form contacts with an axon are retracted (Levine et al., 2001).

The process of oligodendrocyte differentiation and myelination is tightly controlled by various extrinsic and intrinsic factors which can influence proliferation, migration and differentiation of OPCs, their timing and capability to myelinate axonal segments or regulating expression of different genes at certain stages of differentiation. Some of these factors are shown in Figure 1.6. Among those molecules are extrinsic signals and growth factors such as the Notch ligand Jagged, PDGF (Bradl and Lassmann, 2010; Rogister et al., 1999) and morphogens such as Sonic hedgehog (Rogister et al., 1999) that are mainly involved in the generation and maintenance of OPCs. Conversely, intrinsic signaling pathways including PI3K, MAPK, Wnt/ $\beta$-catenin (Colognato et al., 2002; Fancy et al., 2009), their regulated transcriptional factors e.g. Olig1, Olig2, Nkx2.2, Sox10, Tcf4 (Emery, 2010; Fu et al., 2009) and histone acetylation by histone deacetylases (Emery, 2010) mainly control oligodendrocyte 
differentiation. Furthermore, adhesion molecules and proteins of the ECM such as vitronectin, laminin, N-cadherin, NCAM, integrins and their activated signaling pathways can regulate OPC migration and recognition of the unmyelinated axon (Bozzali and Wrabetz, 2004; Bradl and Lassmann, 2010; Colognato et al., 2002; Rogister et al., 1999).

Morover, oligodendrocyte maturation and myelination depends substantially upon axon-glia interactions since transection of the optic nerve or intraocular injection of tetrodoxin which blocks the voltage-gated sodium channels leading to inhibition of the electrical signals from the axon, decreases proliferation of oligodendrocyte precursor cells and therefore prevents myelinogenesis from starting (Barres and Raff, 1993; Demerens et al., 1996). This suggests the existence of signals in or from the axon serving as guidance cues for the myelination process, which is an interesting ongoing topic of research.

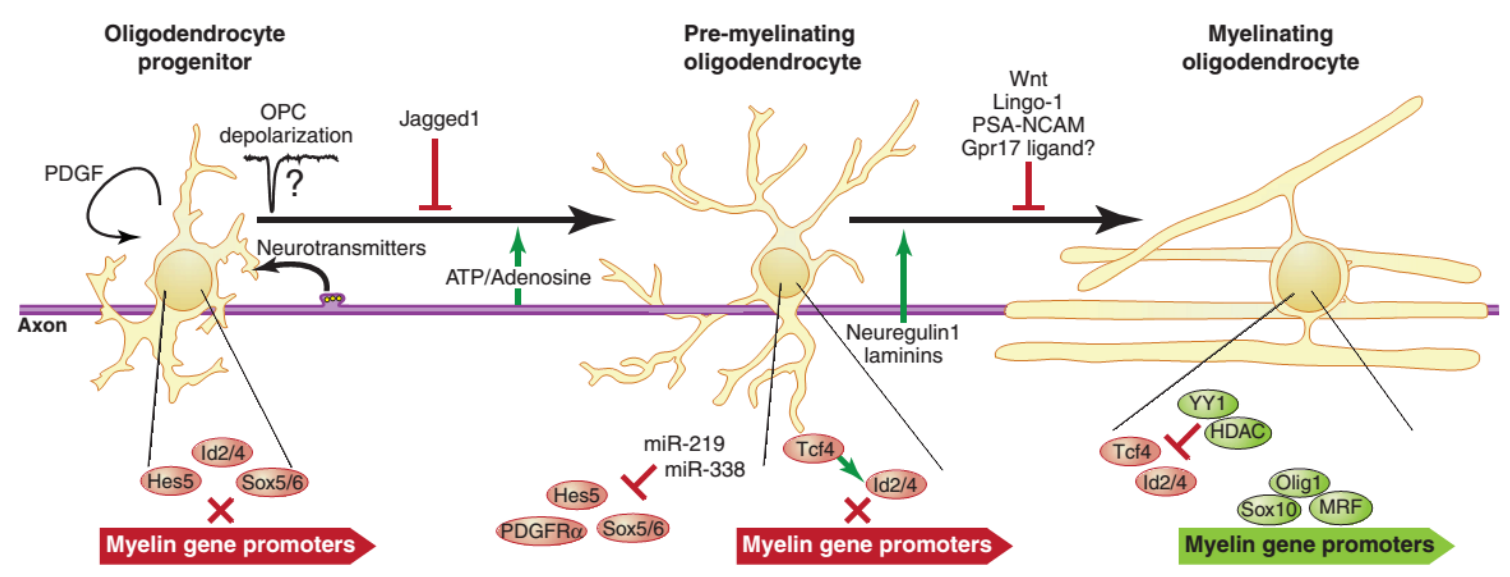

Figure 1.6: Factors influencing oligodendrocyte differentiation and myelination. There are extrinsic signals such as extracellular ligands, secreted molecules and axonal electrical activity; and intrinsic factors including transcriptional regulators and chromatin remodeling. Figure adapted from Emery (2010). Reprinted by permission of Elsevier, license number 3632520535640. 


\subsubsection{Model of myelination in the Central Nervous System (CNS)}

Until recently, the "carpet crawler" was the most accepted model for myelination. It postulates the wrapping of an internode-sized portion of oligodendrocyte membrane around the axon followed by a turn that moves the leading edge underneath the growing sheet (Bunge et al., 1989). However, this model does not explain observations made by other research groups, such as bidirectional ensheathment and unequal thickness of myelin, displaying thicker diameters at the center of the internode (Knobler et al., 1976; Webster, 1971). Furthermore, for this model to apply, very high forces would have to be generated in order to overcome the friction of driving the leading edge to move underneath the myelin layer.

The "liquid croissant" model can explain most of these observations by stating that the oligodendrocyte establishes contact with the axon, its myelin forming process spreads onto the axon similar to dough concomitantly being guided by axonal membrane proteins while new myelin layers are being added on top (Sobottka et al., 2011). This would also explain the previously observed bidirectionality of sheath growth due to the two opposite spiral formations originating from the center. Furthermore, since the center of the sheath is where the oligodendrocyte process started to spread around the axon, the model also accounts for a higher number of layers being temporally generated in the central zone.

Our group (M. Simons) has expanded on the previous models and proposed a more detailed model for myelin wrapping (Figure 1.7). Briefly, the oligodendrocyte process polarizes towards the leading edge after contacting and recognizing the axon. Subsequently, the membrane flattens onto the axon upon which transport of membrane components to the growing zone, also referred as the inner tongue or leading edge, via 
the PI3K signaling pathway is taking place. Radial growth of the myelin sheath then occurs by adding new layers on top of the inner tongue giving rise to a triangular shape with the tip corresponding to the inner tongue and the base to the outermost layer of the sheath. After a few wraps, MBP-dependent compaction is initiated, however, some areas remain uncompacted. These areas are called cytoplasmic channels and function as a bridge connecting the cell body of the myelinating oligodendrocyte with the inner tongue below the compacted layers, allowing the direct transport of material for sheath growth until the completion of myelination, where the majority of cytoplasmic channels are closed. Following sufficient radial extension of the myelin sheath, the layers extend laterally until they attain the full length of the internode and form the paranode and juxtaparanode (Snaidero et al., 2014).

A
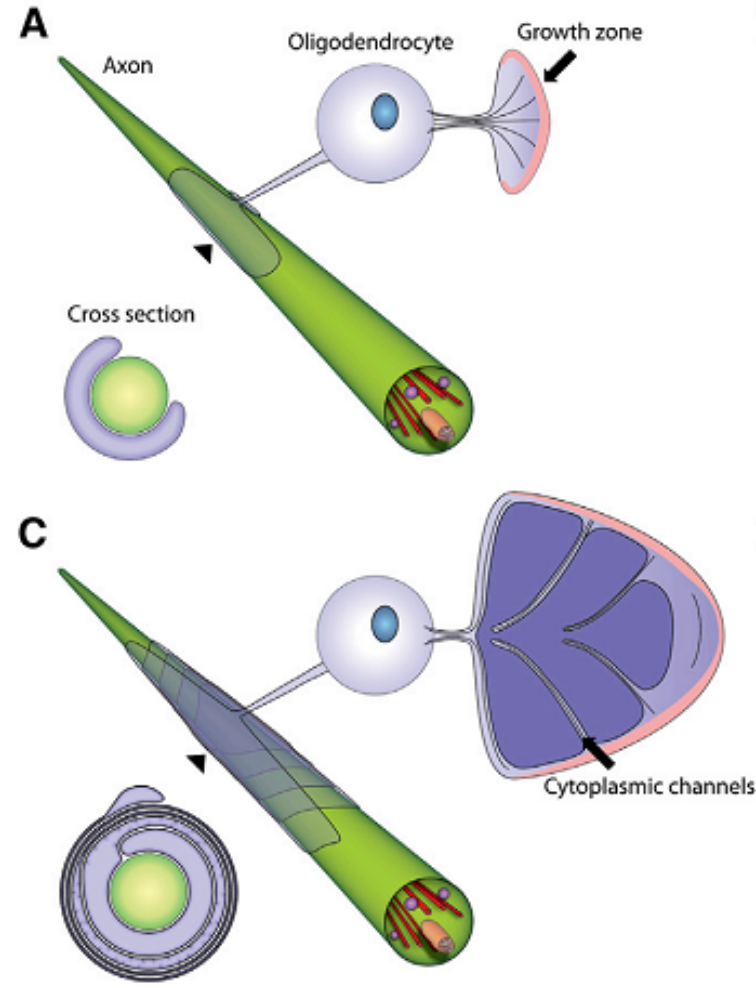

B

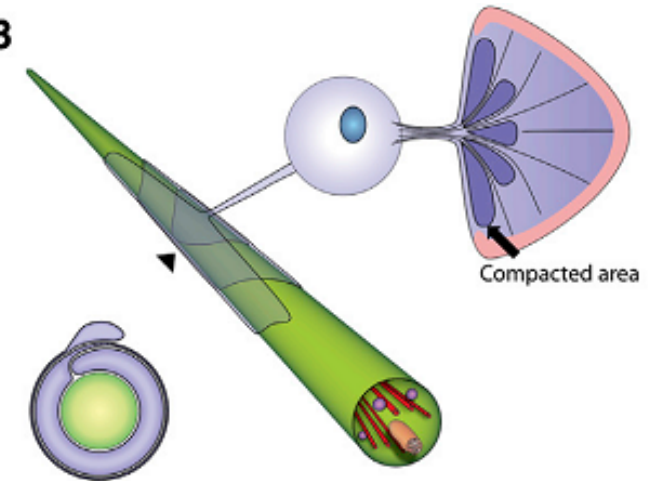

D

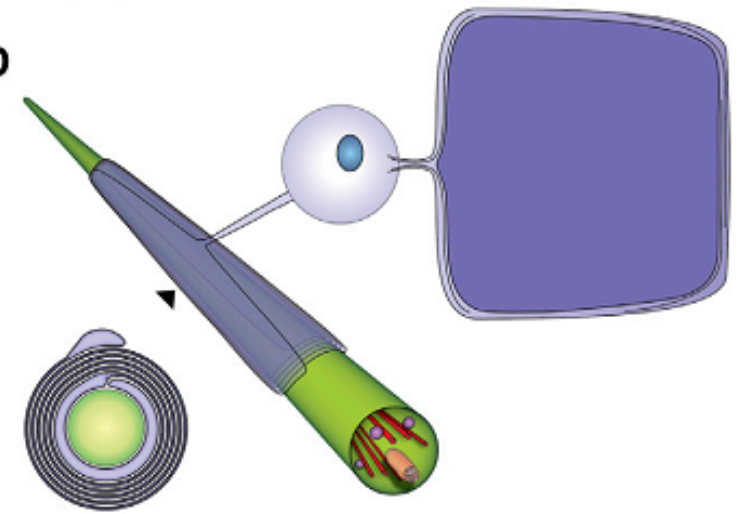

Figure 1.7: Model of myelinogenesis in CNS. Scheme shows how myelination looks like as a crosssection, wrapped and unwrapped view. The axon is shown in green, leading edge in pink, compacted myelin in dark violet and uncompacted myelin in light violet. (A) Initial contact with axon followed by one wrap of uncompacted layer. (B) The inner tongue or leading edge wraps underneath the newly added layers and compaction starts in some areas, there are many cytoplasmic channels connecting both 
extremes. (C) After multiple rounds of wrapping, the inner tongue is still motile and uncompacted, cytoplasmic channels keep the trafficking of components between the cell body and the inner layer. (D) Once the desired thickness has been reached, radial growth is stopped, the layers extend laterally and the cytoplasmic channels are closed. Figure adapted from Snaidero et al. (2014). Reprint by permission of AAAS.

In order to drive the growing leading edge beneath the already formed myelin sheath, considerable amounts of force would need to be generated to constantly propel it forward around the axon. Generally, the major force generator in cells is the cytoskeleton, more specifically motor proteins associated to microtubules mediating transport along the cell, the actoymyosin bundles responsible for contractility and the actin filaments regulating cell shape and motility. Among these cytoskeletal components, actin filaments are the most likely candidates for the force generation in leading edge protrusion due to its known ability to promote forward the membrane movement depending on its polymerization rate.

\subsubsection{Actin filaments and force generation}

Actin filaments also referred to as F-actin are highly concentrated at the cell cortex and mechanically support the stability of the cell. Additionally, they are involved in the generation of different membrane protrusions such as lamellipodia and filopodia, which are essential for cell motility. In muscle cells, together with myosin motors and other proteins, they play a central role in contraction. Actin filaments are polymers consisting of the globular and compacted protein actin, also referred as G-actin that contains an ATP/ADP-binding site and arranges in a right-handed helical structure of 8 $\mathrm{nm}$ in diameter (Alberts et al., 2007). In vertebrates, there are three isoforms of actin. While the $\alpha$-isoform is exclusively expressed in muscle cells, the $\beta$ - and $\gamma$-isoforms are 
found in all non-muscle cells. Since the subunits are asymmetrical, the head-to-tail assembly of filaments gives rise to a polarized structure containing a "barbed" and a "pointed" end. The "barbed" end is also referred as the plus end, due to its rapid polymerization and proximal localization to the cell membrane of the cells. The "pointed end" is also called the minus end due to the slower rates of polymerization and high rates of depolymerization and its localization is towards the center of the cell (Mitchison and Kirschner, 1988).

The actin filament turnover rate (growth at the plus end and shrinkage at the minus end) depends on the availability of the soluble monomers, which in turn are controlled by a number of regulatory proteins. Nucleation factors such as the ARP $2 / 3$ complex, formin and proteins of the WASP family increase the rate of polymerization and induce the branching of actin filaments (Svitkina, 2013). Regulatory proteins binding to free actin monomers are known to promote, e.g. profilin or inhibit, e.g. thymosin and capping proteins, actin filament elongation (Alberts et al., 2007). Disassembly of already assembled filaments is under the direct control of proteins such as $\mathrm{ADF} / \mathrm{Cofilin}$ and gelsoline (Svitkina, 2013). Stabilization of actin filaments can be also achieved by their crosslinking to the ECM and is accomplished by protein complexes containing $\alpha$-actinin, vinculin, filamin and integrins, among others (Hu et al., 2007; Svitkina, 2013). Another level of actin filament regulation is introduced by controlling the activity of the above-mentioned proteins by phosphorilation, binding to phosphatidylinositol 4,5-biphosphate, $\mathrm{pH}$ and signaling pathways involving, for example, Rho GTPases (Ridley, 2011).

One of the main contexts in which cells exert control on polymerization and depolymerization of actin filaments (F-actin) is locomotion. Different types of structures for locomotion are formed at the leading edge with distinct functions 
depending on the associated proteins. These structures can use adhesion-dependent or independent mechanisms to generate the required forces to propel the leading edge forward. The most abundant type of adhesion-dependent cell migration is crawling, which particularly in CNS, is fundamental for neurite extension. Guided movement is achieved by the growth cone, a specialized actin-rich structure. Within the growth cone, two main protrusions are formed, both promoting the forward movement due to F-actin polymerization in the regions of the outer edge of the cell. One of them is the filopodium, a finger-like continuation of the plasma membrane formed by parallel bundles of actin filaments, which extend from the leading edge in an exploratory manner (Chhabra and Higgs, 2007; Ridley, 2011). The other structure is the lamellipodium, a very thin, sheet-like region at the leading edge of the growth cone formed by branched actin filaments arranged in an interconnected, "dendritic" meshwork (Ridley, 2011; Svitkina, 2013). The lamellipodium is highly dynamic and located distally at the leading edge. It is followed by a more stable region, the lamellum, which extends into the cell body and couples the actin network to myosin contractility and attachment to the extracellular substrate (Chhabra and Higgs, 2007; Ridley, 2011). Movement is generated by a treadmill mechanism in which the addition of actin monomers at the plus end as well as the contractile properties of myosin pulling the actin filaments towards the cell body generates a retrograde flow. Focal adhesion contacts that are composed of a molecular cluster of proteins, attach the filaments to the substrate thus allowing the transduction of the generated traction forces resulting in the pushing forward of the leading edge (Chhabra and Higgs, 2007; Hu et al., 2007; Mitchison and Kirschner, 1988; Renkawitz and Sixt, 2010).

On the other hand, an example for adhesion-independent mechanisms is locomotion driven solely by actin polymerization, independent of myosin motors and 
adhesion to the substrate, which is also referred to as amoeboid migration. This type of locomotion is mainly used by leukocytes when they have to squeeze in between the epithelial cells of the blood vessels. The principle is a cell deformation that inflates the leading edge depending on actin filaments polymerization in a $3 \mathrm{D}$ environment. Confinement is required for the protrusion to intercalate between tight spaces and thus generate an asymmetric friction, which is higher at the leading edge. Forces generated by friction are responsible for pushing the cell after the rear end contracts (Renkawitz and Sixt, 2010). 


\subsection{Aims of the Project}

Until now, mechanistic details of myelin wrapping, in particular how the innermost layer is driven around the axon in a highly confined area, are poorly understood. The main aim of this project was to elucidate and describe the driving force for leading edge protrusion, myelin wrapping and myelin membrane flattening. Since oligodendrocytes undergo a series of morphological changes in order to generate the myelin sheath, we hypothesized that its mechanical properties must also change during their differentiation. To experimentally prove this, having the appropriate tools is crucial. Hence, a vertical optical trap has been designed for this purpose and the methodological verification is presented in chapter 3.1. Subsequently, in chapter 3.2, I present an interdisciplinary approach including live imaging confocal microscopy, mouse models, optical trapping and other biophysical measurements to study how actin dynamics influence leading edge protrusion and membrane flattening in myelin sheath formation. 


\section{Materials and Methods $\mid 2$}

\subsection{Materials}

2.1.1 Chemicals and consumables

All chemicals were obtained from Sigma-Aldrich (Sigma-Aldrich Chemie GmbH, Munich, Germany) or Merck Millipore (Merck KGaA, Darmstadt, Germany) unless stated otherwise.

All basal media, supplements, antibiotics and sera for cell culture were purchased from Gibco (Life technologies GmbH, Darmstadt, Germany) unless specified.

Consumables and cell culture plates were purchased from Greiner bio-one (Greiner bio-one GmbH, Frickenhausen, Germany) and Eppendorf (Eppendorf AG, Hamburg, Germany). Imaging dishes for live imaging were purchased from ibidi (ibidi $\mathrm{GmbH}$, Munich, Germany).

Micro cantilevers were purchased from Olympus (Asylum Research, Mannheim, Germany) and colloidal probes were obtained from NanoAndMore (NanoAndMore $\mathrm{GmbH}$, Wetzlar, Germany). Microspheres were purchased from Bangs Laboratories, Inc (Polysciences Europe GmbH, Eppelheim, Germany). 
All optical components for building the vertical optical trap were purchased at Thorlabs (Thorlabs GmbH, Munich, Germany) unless otherwise specified.

\subsubsection{Antibodies}

Primary antibodies are listed in Table 2.1. Secondary conjugated antibody fluorophores were purchase from Dianova (Dianova GmbH, Hamburg, Germany).

Table 2.1: Summary of antibodies used in this study.

\begin{tabular}{llll}
\hline Target & $\begin{array}{c}\text { Host } \\
\text { species }\end{array}$ & Application & \\
& & & \\
CC1 & Mouse IgG2b & IHC (1/100) & Calbiochem-Merck Millipore, Darmstadt, Germany \\
MBP & Rabbit & IF/IHC (1/300) & Dako Deutschland GmbH, Hamburg, Germany \\
Nkx2.2 & Mouse IgG2b & IHC (1/5) & Developmental studies hybridoma bank, Iowa, USA \\
O1 & Mouse IgM & IF (1/100) & Self made from hybridoma (Kuhlmann-Krieg et al., 1988; Raff et al., 1983) \\
O4 & Mouse IgM & IF (1/100) & Self made from hybridoma (Kuhlmann-Krieg et al., 1988; Raff et al., 1983; \\
& & & Trotter and Schachner, 1989) \\
Olig2 & Rabbit & IHC (1/250) & Merk Millipore, Darmstadt, Germany \\
Vinculin & Mouse IgG1 & IF (1/400) & Sigma-Aldrich, Munich, Germany \\
\hline
\end{tabular}

\subsubsection{Primers}

The primers used in this study were synthesized in the DNA core facility, the AGCT-laboratory of the Max Planck Institute for Experimental Medicine (Göttingen, Germany). The list of primers used for genotyping is shown in Table 2.2. 
Table 2.2: Summary of primers used for genotyping.

\begin{tabular}{|c|c|c|c|}
\hline Gen & PCR & Number & Sequence \\
\hline \multirow[t]{3}{*}{ Cofilin-1 } & CFL1FX & 27596 & 5- CGCTGGACCAGAGCACGCGGCATC -3' \\
\hline & & 27597 & 5'-CTGGAAGGGTTGTTACAACCCTGG -3' \\
\hline & & 27598 & 5'- CATGAAGGTTCGCAAGTCCTCAAC -3' \\
\hline \multirow[t]{3}{*}{$\mathrm{ADF}$} & ADF-KO & 28830 & 5'- GATTAAGTTGGGTAACGCC -3' \\
\hline & & 28831 & 5'- GAAGAAGGCAAAGAGATCTT -3' \\
\hline & & 28832 & 5'- CTACCTAAAGGGCATCCTTTC -3' \\
\hline \multirow[t]{3}{*}{ CNCE-Cre } & CNCE-GNT & 01955 & 5'- CATAGCCTGAAGAACGAGA -3' \\
\hline & & 02016 & 5'- GCCTTCAAACTGTCCATCTC -3' \\
\hline & & 07315 & 5'- CCCAGCCCTTTTATTACCAC -3' \\
\hline \multirow[t]{2}{*}{ PLP1-Cre ERT2 } & PLP-Cre & 10099 & 5'- TGGACAGCTGGGACAAAGTAAGC -3' \\
\hline & & & 5'- CGTTGCATCGACCGGTAATGCAGGC -3' \\
\hline
\end{tabular}

\subsubsection{General buffers and solutions}

Phosphate Buffered Saline (PBS):

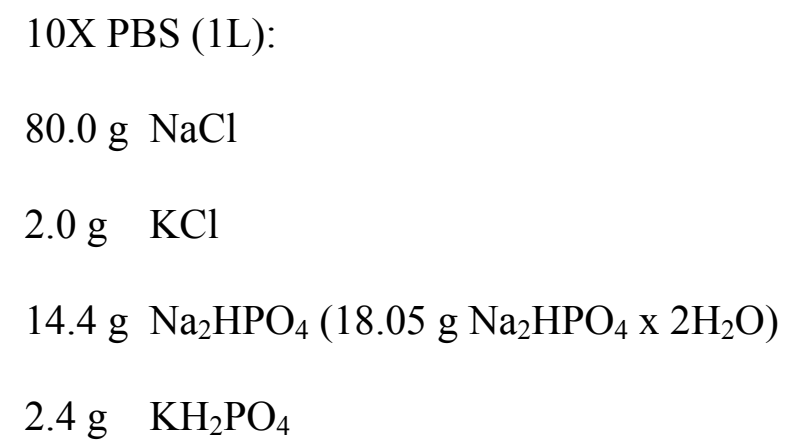

Dissolve in $\mathrm{dH}_{2} \mathrm{O}$; adjust $\mathrm{pH}$ value to 7.4 and autoclave.

To obtain 1X PBS, 10X PBS was diluted 10 times with $\mathrm{dH}_{2} \mathrm{O}$.

Tris-Acetate-EDTA (TAE) buffer:

50X TAE (1L):

$242 \mathrm{~g}$ Tris base 
$57.1 \mathrm{ml}$ Glacial acetic acid

$100 \mathrm{ml} \mathrm{0.5 \%} \mathrm{EDTA}$

Dissolve in $\mathrm{dH}_{2} \mathrm{O}$ and adjust $\mathrm{pH}$ 8.5.

To obtain $1 \mathrm{X}$ working solution, 50X TAE was diluted 50 times with $\mathrm{dH}_{2} \mathrm{O}$.

Krebs Ringer Solution:

$\begin{array}{ll}120 \mathrm{mM} & \mathrm{NaCl} \\ 4.7 \mathrm{mM} & \mathrm{KCl}, \\ 1.2 \mathrm{mM} & \mathrm{CaCl}_{2} \\ 0.7 \mathrm{mM} & \mathrm{MgSO}_{4} \\ 10 \mathrm{mM} & \text { Glucose } \\ 10 \mathrm{mM} & \text { HEPES }\end{array}$

Dilute in $\mathrm{dH}_{2} \mathrm{O}$, adjust $\mathrm{pH}$ value to 7.4 and autoclave. Keep at $4{ }^{\circ} \mathrm{C}$ for no longer than 2 weeks.

Super SATO medium (100 ml):

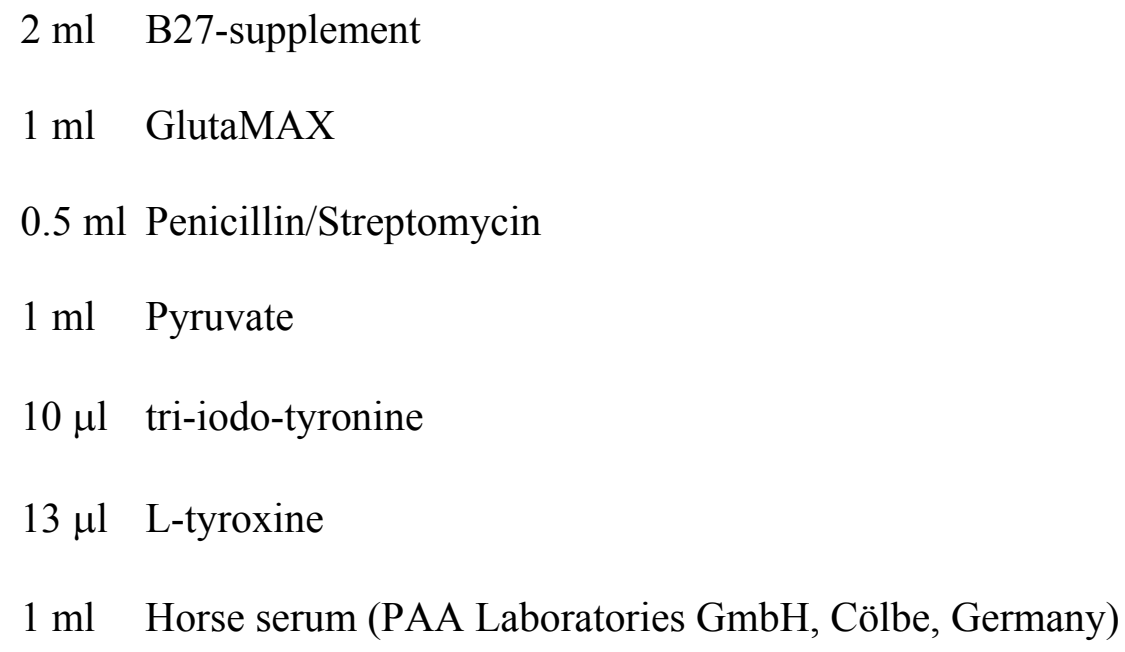

Dilute in DMEM $4500 \mathrm{mg} / \mathrm{L}$ glucose, filter and keep at $4{ }^{\circ} \mathrm{C}$ for no longer than 2 weeks. 
BME medium (500 ml):

$50 \mathrm{ml}$ Horse serum (PAA Laboratories GmbH, Cölbe, Germany)

$2.5 \mathrm{ml}$ Penicillin/Streptomycin

$5 \mathrm{ml}$ GlutaMAX

Dilute in Basal Medium Eagle (BME). Keep at $4{ }^{\circ} \mathrm{C}$.

PLL solution $100 \mu \mathrm{g} / \mathrm{ml}(500 \mathrm{ml})$ :

Dilute $5 \mathrm{ml}$ 50X Poly-L-Lysine stock (Mw>300000, Sigma-Aldrich) in sterile PBS. Keep at $4{ }^{\circ} \mathrm{C}$.

4\% Paraformaldehyde (PFA) solution (200 ml):

$16 \%$ PFA (100 ml):

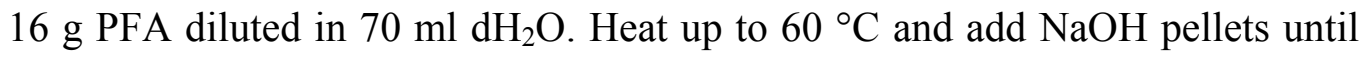
the solution becomes clear. Add $10 \mathrm{ml}$ of $10 \mathrm{X}$ PBS, adjust $\mathrm{pH}$ to 7.4 and adjust volume to $100 \mathrm{ml}$ with $\mathrm{dH}_{2} \mathrm{O}$. Aliquot and store at $-20^{\circ} \mathrm{C}$.

4\% working solution was prepared freshly by diluting $50 \mathrm{ml}$ of PFA stock solution in $150 \mathrm{ml} 1 \mathrm{X} \mathrm{PBS}$, filtered and stored at $4{ }^{\circ} \mathrm{C}$ for no more than a week.

For Immunofluorescence of cultured cells, $25 \mu \mathrm{l}$ of $25 \%$ Glutaraldehyde (Merck-Millipore) was added to $2 \mathrm{ml}$ of 4\% PFA (when indicated).

Blocking solution (100X):

$\begin{array}{ll}2 \% & \text { Fetal bovine serum } \\ 2 \% & \text { BSA } \\ 2 \% & \text { fish gelatin }\end{array}$


Dilute in $1 \mathrm{X}$ PBS, filter and store at $-20{ }^{\circ} \mathrm{C}$. For diluting antibodies, a $10 \%$ working solution was prepared by diluting the $100 \mathrm{X}$ solution 10 times in $1 \mathrm{X}$ PBS.

Mowiol solution:

Stir $2.4 \mathrm{~g}$ mowiol, $6 \mathrm{~g}$ glycerol and $6 \mathrm{ml} \mathrm{H}_{2} \mathrm{O}$ for several hours at room temperature. Add $12 \mathrm{ml} 0.2 \mathrm{M}$ Tris- $\mathrm{HCl}\left(\mathrm{pH} \mathrm{8.5)}\right.$ and incubate at $60{ }^{\circ} \mathrm{C}$ for 10 min. Centrifuge at $4000 \mathrm{~g}$ for $15 \mathrm{~min}$. Aliquot and keep at $-20{ }^{\circ} \mathrm{C}$.

14\% Chloral hydrate:

Dissolve $1.4 \mathrm{~g}$ of Chloral hydrate C-IV (Sigma-Aldrich) in $10 \mathrm{ml}$ of PBS. Store at room temperature. Use $50-100 \mu 1$ for pups and $200-250 \mu 1$ for older animals.

4\% Paraformaldehyde (PFA) solution for EM (200 ml in Phosphate buffer) 2X Phosphate buffer (PB):

$0.72 \mathrm{~g} \mathrm{NaH}_{2} \mathrm{PO}_{4}$

$6.2 \mathrm{~g} \quad \mathrm{Na}_{2} \mathrm{HPO}_{4} \times 2 \mathrm{H}_{2} \mathrm{O}$

$2 \mathrm{~g} \quad \mathrm{NaCl}$

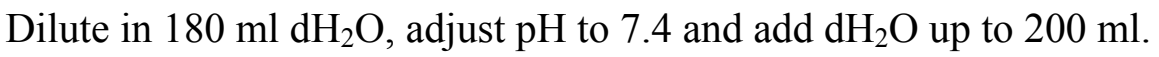

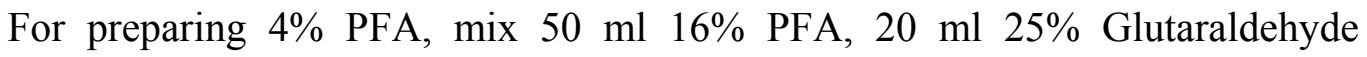

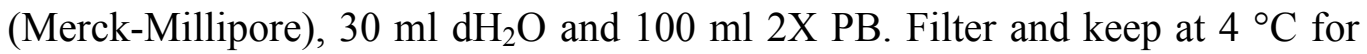
no longer than 1 week. 


\subsubsection{Software}

The software used for data acquisition and processing is listed in Table 2.3.

Table 2.3: Summary of software used in this study.

\begin{tabular}{lll}
\hline \multicolumn{1}{c}{ Software } & \multicolumn{1}{c}{ Application } & \multicolumn{1}{c}{ Source/Manufacturer } \\
& & \\
Adobe Illustrator CS3 & Figure making & Adobe Systems, Inc \\
GraphPad PRISM 5 & Statistical analysis and graph production & www.graphpad.com \\
ImageJ/Fiji (Free) & Image processing and analysis & www.fiji.sc \\
Igor Pro 6.22A & AFM controller, data acquisition and processing & www.wavemetrics.com/ www.asylumresearch.com \\
LabVIEW & Optical trap controller, data acquisition and analysis & National Instruments Germany GmbH \\
LAS AF & Live imaging and confocal images acquisition & Leica Microsystems, Mnanheim, Germany \\
LAS AF 3.1.0 & Fluorescent images acquisition & Leica Microsystems, Mannheim, Germany \\
\hline
\end{tabular}




\subsection{Methods}

\subsubsection{Atomic Force Microscopy}

AFM indentation experiments were carried out using an MFP-3D (Asylum Research, Santa Barbara, CA, USA) that was mounted on a custom built inverted optical microscope using an oil immersion objective $(60 \times 1.45 \mathrm{NA}$ plapon objective, Olympus, Japan). A $25 \mathrm{~mm}$ diameter coverslip containing the cells was mounted in an open sample chamber and filled with $350 \mu \mathrm{l}$ Krebs Ringer solution. First, a cell was selected using the optical microscope. The AFM tip was then brought down to indent the cell, recording the motion of the z-piezo and the applied force. Calibration was done as described in section 1.1.3.1 by fitting the power spectrum to a simple harmonic oscillator. When performing measurements on cells, deformation was computed from the displacement of the z-piezo minus the bending of the cantilever.

For cell indentation experiments, cantilevers with a $1.98 \mu \mathrm{m}$ diameter bead glued to the end ( $k=0.08 \mathrm{~N} / \mathrm{m}$, CP-PNPL-PS, NanoAndMore) were used and the force applied to the cells was set up to $1 \mathrm{nN}$. The Young's modulus of the cells was obtained by fitting the indentation curves with the Hertz contact mechanics model. This model describes the contact between two spherical elastic bodies. It assumes (i) that the contact area is much smaller than the radius of curvature of the surfaces; (ii) that there are no adhesive forces between the probe and the indented surface; (iii) that the materials are homogeneous and with elastic properties (Hertz, 1881). 
In this particular case the cell is modeled as an elastic half space (a sphere with an infinitely large radius) and the Young's modulus of the probe is assumed to be much higher than that of the cell, and the Hertz equation can be simplified to:

$$
F=\frac{4}{3} \frac{E}{\left(1-v^{2}\right)} \sqrt{R_{b}} d_{z}^{3 / 2}
$$

The force $(F)$ increases exponentially with the indentation $\left(d_{z}\right)$ and depends on the cell's Young's modulus $(E)$, the bead radius $\left(R_{b}\right)$ and the Poisson ratio of the cell $(v)$.

It is also possible to estimate the Young's modulus from stretching experiments by using a variation of Eq. 9 describing the deformation of an elastic half space with a disk-shaped contact area. In this case, assuming that the contact radius remains constant (calculated based on the maximal indentation during the preceding indentation experiments), there will be a linear relation between force and indentation.

$$
F=\frac{E}{\left(1-v^{2}\right)} R_{c} d_{z}
$$

$R_{c}$ is the contact radius, which since the indenter is a sphere, will increase with the indentation according to:

$$
R_{c}=\sqrt{d_{z} R_{b}}
$$

As was already discussed in chapter 1.1 .2 cells are neither homogenous in structure nor behave as an ideal elastic material. As a consequence the reported Young's moduli do not represent an intrinsic mechanical parameter of the cell but will in part depend on the experimental conditions, most notably the deformation rate and the indentation depth as will be shown in chapter 3.1. To circumvent this issue we refer to the calculated Young's modulus as the apparent cell's Young's modulus.

For force mapping and tether pulling, v-shaped cantilevers $(k=0.03 \mathrm{~N} / \mathrm{m}, \mathrm{BL}$ RC150VB-HW, Olympus) were used, the applied force was limited to $300 \mathrm{pN}$ and the 
probe was in contract with the cell for $1 \mathrm{~s}$ before pulling at $3.2 \mu \mathrm{m} / \mathrm{s}$ for a distance of 10 $\mu \mathrm{m}$. Mapping was done on an array of 20x20 points covering an area of $80 \times 80 \mu \mathrm{m}$. The tether force $\left(T_{F}\right)$ was quantified as the force required to further extend a tether or to hold a tether at a constant length, depending on the experiment. See also section 1.1.2.3.

\subsubsection{Optical trap}

The optical trap used to indent the cells at low force was built around a commercial upright microscope body (Eclipse 50i Nikon, Japan) and set up for trapping and detection vertical to the coverslip as described in (Bodensiek et al., 2013; Nawaz et al., 2012). Briefly, $974 \mathrm{~nm}$ laser light emitted from a $300 \mathrm{~mW}$ single mode fiber was collimated and combined with the optical path using a dichroic mirror and focused onto the sample through a water immersion objective $(60 \times 1.27 \mathrm{NA}$ Plan Apo IR objective, Nikon, Japan). A closed-loop objective piezo element (P-721, Physik Instrumente $\mathrm{GmbH}$, Karlsruhe, Germany) was used to move the objective up and down. The displacement of the bead from the trap center was monitored using a quadrant photodetector (S5980, Hamamatsu Photonics Deutschland GmbH, Germany) and trap stiffness calibration was done as described in section 1.1.3.2 by fitting a Lorentzian function to the power spectrum. The microscope is also equipped with a blue LED and a CCD camera to allow optical visualization of the sample while measuring.

Sample preparation was done by placing the coverslip containing the cells on a closed sample chamber, consisting of a microscope slide and a $100 \mu \mathrm{m}$ thick spacer (Parafilm) and adding Krebs Ringer solution containing polystyrene beads of $0.76 \mu \mathrm{m}$ diameter (Bangs laboratories). 
For all the experiments the force applied to the cells was set to $10 \mathrm{pN}$. For tether pulling the bead was in contact with the cell for $1-2 \mathrm{~s}$ before pulling at $4 \mu \mathrm{m} / \mathrm{s}$ for a maximum distance of $80 \mu \mathrm{m}$.

Young's moduli $(E)$ and Tether force $\left(T_{F}\right)$ were extracted from the curves as described in the previous section.

\subsubsection{Cell line culture}

All cell culture work was carried out according to security level S1 safety rules. Cell incubation was carried out in humidified, $37{ }^{\circ} \mathrm{C}, 5 \% \mathrm{CO}_{2}$ incubators. NIH3T3 mouse embryonic fibroblasts (DSMZ, Braunschweig, Germany) were grown in Dulbecco's modified Eagle's medium (DMEM) with $4.5 \mathrm{~g} / \mathrm{L}$ glucose supplemented with 10\% FBS, and Penicillin/Streptomycin. At least $24 \mathrm{~h}$ before experiments, cells were split with $0.25 \%$ Trypsin/EDTA and seeded onto Poly-L-Lysine-coated coverslips. For disruption of the actin cortex, cells were incubated with $1 \mu \mathrm{M}$ latrunculin-A (Calbiochem-Merck Millipore, Darmstadt, Germany) for 30 min and measured directly afterwards. All AFM and OT experiments were performed at room temperature.

\subsubsection{Primary Oligodendrocyte culture}

As described before, everything was carried out according to S1 safety rules. Incubations were carried out in humidified, $37^{\circ} \mathrm{C}, 5 \% \mathrm{CO}_{2}$ incubators. Primary cultures were prepared from postnatal day 0-2 (P0-P2) mice. In brief, mice were decapitated and the brain was extracted. Olfactory bulb, cerebellum and meninges were removed and 
brain hemispheres were digested with $0.25 \%$ Trypsin-EDTA for $5 \mathrm{~min}$ at $37{ }^{\circ} \mathrm{C}$. Subsequently the brain hemispheres were dissociated in BME medium supplemented with $10 \%$ HS, Penicillin/Streptomycin and GlutaMAX. The cell suspension was filtered using a pore size of $0.2 \mu \mathrm{m}$ and plated onto Poly-L-Lysine-coated flasks with BME medium (supplemented with 10\% HS, GlutaMAX and Penicillin/Streptomycin) and Super SATO medium (DMEM 4.5 g/L glucose, 1\% HS, B27 supplement, GlutaMAX, sodium pyruvate, triiodotyronine, L-tyroxine and Penicillin/Streptomycin). This glial mixed culture was incubated for $7-9$ days and eventually consisted of two main types of cells: the bottom of the flask contained a monolayer of astrocytes with oligodendrocyte progenitor cells growing on top of them. Residual microglial cells were constantly removed by gently tapping the flasks and changing parts of the medium.

For a highly pure oligodendrocyte progenitor culture, cells were separated using mechanical dissociation by shaking the flasks to detach the oligodendrocytes whereas astrocytes remain attached to the bottom of the flask. The medium was collected and centrifuged at $0.9 \mathrm{rpm}$ for 10 minutes followed by resuspension of progenitors in Super SATO medium and incubation in a petri dish for 2 min to allow clustered and other types of cells to adhere at the bottom of the plate. Subsequently, the cell suspension was collected from the plate and cells plated onto Poly-L-Lysine-coated surfaces (100 $\mu \mathrm{g} / \mathrm{ml})$ with Super SATO medium followed by a differentiation period between 6 hours and 5 days. The cell density plated onto each well was 15000 for 96-well plates, 1500020000 for 48-well plates, 20000-40000 for 24-well plates and 40000 for imaging dishes.

For depolymerization of F-actin, cells were treated with $10 \mu \mathrm{M}$ latrunculin-A (Calbiochem) and incubated for 30 min prior to measurements. To stabilize F-actin, cells were incubated with $200 \mathrm{nM}$ Jasplakinolide (Calbiochem). For cell-substrate detachment, cells were treated with $200 \mu \mathrm{g} / \mathrm{ml}$ of Trypsin (Gibco) for 10-15 minutes. 


\subsubsection{Immunofluorescence (IF)}

Cells were fixed in 4\% PFA + Glutaraldehyde $(25 \mu 1$ of $25 \%$ Glutaraldhehyde for $2 \mathrm{ml} \mathrm{4 \%}$ PFA) for $10 \mathrm{~min}$ at room temperature, washed 5 times with PBS, permeabilized with $0.1 \%$ Triton X-100 (in PBS) for 2 min and washed 5 times with PBS. To reduce unspecific binding of the antibody, coverslips were incubated for 30 min with 100X blocking solution (2\% fish gelatin, 2\% FCS, 2\% BSA in PBS) followed by incubation with primary antibody diluted in $10 \%$ blocking solution for $1 \mathrm{~h}$ at room temperature. After washing 5 times with PBS, secondary antibody diluted in $10 \%$ blocking solution was incubated for $40 \mathrm{~min}$ at room temperature. Coverslips were washed 5 times with PBS and 1 time with $\mathrm{dH}_{2} \mathrm{O}$ before mounting on a drop of Mowiol mounting medium.

The primary antibodies used are listed on table 2.1. For Vinculin staining cells were fixed only with 4\% PFA. For plasma membrane associated antibodies (O1 and O4), coverslips were blocked and incubated with primary antibody immediately after fixation. After washing steps, cells were fixed with 1\% PFA for 5 min and washed 3 times with PBS. The rest of the protocol was followed as described above.

The fluorophore-conjugated secondary antibodies used were: Alexa 488 (anti mouse and rabbit), 555 (anti mouse), 568 (anti rabbit) and 647 (anti mouse and rabbit) (1/2000). For labeling of G-actin, Alexa Fluor 488-coupled Desoxyribonuclease I (LifeTechnologies) 1/200 was used and for labeling F-actin, phalloidin-rodamine or Alexa Fluor 488 phalloidin (LifeTechnologies) 1/300 were used.

The imaging was performed with an epifluorescence microscope (LEICA) using a $40 \times$ objective. 


\subsubsection{Immunohistochemistry (IHC)}

Animals were anesthetized intraperitoneally using 14\% Chloral hydrate. Intracardiac perfusion was carried out by inserting a needle into the left ventricle and cutting the right atrium. To wash out the blood, ice cold sterile PBS was pumped in by a mini-peristaltic pump (Harvard Apparatus) and perfusion was done in fresh 4\% PFA followed by postfixation of the dissected tissue over night (brain and spine). Spinal cords were dissected from the spine and kept together with brains in 30\% Sucrose solution (in PBS) until sinking down for 1-3 days and being frozen afterwards using Tissue-Tek O.C.T. (Sakura Finetek Germany GmbH, Staufen, Germany) on dry ice and stored at $-80{ }^{\circ} \mathrm{C}$. Sections between the cervical and thoracic area were cut using a cryostat (Research Cryostat Leica CM3050 S) directly onto glass slides (Thermo Scientific) and stored at $-20{ }^{\circ} \mathrm{C}$. For staining, sections were washed 3 times for $5 \mathrm{~min}$ with PBS, permeabilized with $0.5 \%$ Triton X-100 (in PBS) for $1 \mathrm{~h}$ and incubated with blocking solution (2\% FCS, $2 \%$ fish gelatin and 2\% BSA in PBS) for 1 h. Primary antibodies were diluted in $10 \%$ blocking solution and incubated as described below, washed 5 times for 5 minutes with PBS $+0.2 \%$ Tween $(\mathrm{CC} 1)$ or $0.3 \%$ Triton $\mathrm{X}-100$ (Nkx2.2) followed by incubation with respective secondary antibodies for $1 \mathrm{~h}$ at room temperature. After washing 5 times with PBS-Tween or PBS-Triton X-100 for 5 min and once with $\mathrm{dH}_{2} \mathrm{O}$, sections were mounted with Mowiol solution. For imaging, a Leica DMI6000 inverse microscope or a Leica SP5 (Mannheim, Germany) confocal laser scanning microscope (CLSM) were used.

F- and G-actin staining (on $25 \mu \mathrm{m}$ sections) were done overnight at $4{ }^{\circ} \mathrm{C}$ using Rhodamine phalloidin 1/500 and Alexa Fluor 488 DNase I 1/200. For $10 \mu \mathrm{m}$ sections Olig2 1/250 (Millipore), Nkx2.2 1/5 (Developmental Studies Hybridoma Bank at the 
University of Iowa) and CC1 1/100 (Calbiochem) antibodies were incubated for two nights at $4{ }^{\circ} \mathrm{C}$.

For the combined $\mathrm{CC} 1 / \mathrm{Olig} 2$ staining, antigen retrieval was carried out before permeabilization by autoclaving the slides submerged in $10 \mathrm{mM}$ Sodium Citrate buffer, pH 6 followed by cooling down in room temperature PBS.

\subsubsection{Interference Reflection Microscopy (IRM)}

For IRM imaging, cells were monitored using a Leica SP5 (Mannheim, Germany) confocal laser scanning microscope with a 40× (NA 1.25) objective and a laser illumination to minimize phototoxicity $(633 \mathrm{~nm})$. The reflected light was collected using a photomultiplier tube detector every 17 or 34 seconds. 2 cells were imaged simultaneously on each imaging dish at $37{ }^{\circ} \mathrm{C}$ (imaging medium was Super SATO medium +25 mM HEPES) .

The IRM time series were semi-automatically analysed with a custom-written FIJI macro (written by Ioannis Alexopoulos and Mišo Mitkovski). Briefly, a "walking difference" routine was applied, which revealed pixels that reported dynamic changes in cell-surface adhesion. Pixel intensities above a threshold, which was kept constant for all experiments, were used in the cell-surface adhesion quantification. Additionally, the cell footprint area was quantified after segmentation of the IRM signal with the Trainable Weka Segmentation plugin of FIJI. The dynamic were quantified by calculating the slope of the dynamic pixels over a given time and the adhesion was quantified using Weka Segmentation to separate the adhesive pixels (dark grey and black). In both cases the data was normalized to the cell footprint area. 


\subsubsection{Mice}

All animal experiments were performed according to the Lower Saxony State regulations for animal experimentation. Mutant mice were bred and maintained on a C57BL/6 background. Cnp-Cre (Lappe-Siefke et al., 2003), Plp1-CreERT2 (Leone et al., 2003), ADF KO and Cofilin1 ${ }^{\mathrm{fl} / \mathrm{fl}}$ mice have been described previously (Bellenchi et al., 2007; Flynn et al., 2012). To generate oligodendrocyte-specific ADF/Cofilin1 double knockout animals (AC DKO), Cnp-Cre mice were cross-bread with Cofilin $1^{\mathrm{fl} / \mathrm{fl}}$ and then crossed with ADF KO animals to generate $\mathrm{ADF}^{-/-} \mathrm{Cofilin} 1^{\mathrm{fl} / \mathrm{fl}} \mathrm{Cnp} 1-\mathrm{Cre}^{+/-}$mice. To generate the inducible ADF/Cofilin1 double knock out $\left(\mathrm{ADF}^{-/-}\right.$Cofilin1 ${ }^{\mathrm{fl} / \mathrm{fl}} \mathrm{Plp} 1$ CreERT2), Cofilin1 ${ }^{\mathrm{fl} / \mathrm{fl}}$ animals were crossed with Plp1-CreERT2 before breeding with ADF KO mice.

Tamoxifen (Sigma-Aldrich) was dissolved in corn oil (Sigma-Aldrich) at a final concentration of $10 \mathrm{mg} / \mathrm{ml}$. $\mathrm{ADF}^{-/-}$Cofilin1 ${ }^{\mathrm{fl} / \mathrm{fl}} \mathrm{Plp} 1-\mathrm{CreERT} 2$ mice at P21 were injected intraperitoneal with $100 \mu \mathrm{g}$ tamoxifen per gram of mouse weight once a day for 5 consecutive days. As controls, animals without expression of Cre were used. The recombination efficiency of the treatment is at least $80 \%$ as described by (Leone et al., 2003).

\subsubsection{Genotyping}

A small fraction of the animal's tail was cut and genomic DNA was extracted using the Invitek Smarter Nucleic Acid Sample Preparation Kit (STRATEC Molecular $\mathrm{GmbH}$, Berlin, Germany) according to manufacturer's instructions and the following modifications. Tails were digested overnight with $400 \mu \mathrm{l}$ of Lysis buffer plus $15 \mu 1$ 
Proteinase $\mathrm{K}$ at $52{ }^{\circ} \mathrm{C}$ and shaking at $400 \mathrm{rpm}$. Final centrifugation to eliminate traces of ethanol was done at $11000 \mathrm{rpm}$ for 6 min and elution of DNA was done in $100 \mu \mathrm{l}$ of elution buffer.

For genotyping, the DNA was amplified by polymerase chain reaction (PCR) using GoTaq DNA polymerase (Promega, Manheim, Germany) and the set of primers listed on table 2.2. The reaction mix was prepared as follows:

$11.4 \mu \mathrm{lddH} 2 \mathrm{O}$

$4 \mu \mathrm{5X}$ Buffer

$1 \mu \mathrm{l} 25 \mathrm{mM} \mathrm{MgCl} 2$

$1 \mu \mathrm{l} \quad 10 \mathrm{mM}$ dNTP (diluted 1/5)

$0.5 \mu \mathrm{l} 50 \mathrm{pmol} / \mu 1$ Primer 1 (diluted $1 / 5)$

$0.5 \mu \mathrm{l} 50 \mathrm{pmol} / \mu \mathrm{l}$ Primer $2($ diluted $1 / 5)$

$0.5 \mu \mathrm{l} 50 \mathrm{pmol} / \mu 1$ Primer 3 (diluted $1 / 5$ )

$0.1 \mu \mathrm{l} 5 \mathrm{u} / \mu \mathrm{l}$ GoTaq DNA polymerase

$1 \mu 1 \quad$ DNA

The reaction was carried out in a T3000 Thermocycler Kombi (Biometra) using the programs described in table 2.4 .

Table 2.4: PCR programs used for genotyping.

\begin{tabular}{|c|c|c|c|}
\hline CFL1FX & ADF-KO & CNCE-GNT & PLP-Cre \\
\hline $94^{\circ} \mathrm{C} \quad 2 \mathrm{~min}$ & $94{ }^{\circ} \mathrm{C} \quad 2 \mathrm{~min}$ & $95^{\circ} \mathrm{C} \quad 3 \mathrm{~min}$ & $96{ }^{\circ} \mathrm{C} \quad 3 \mathrm{~min}$ \\
\hline $94{ }^{\circ} \mathrm{C} \quad 30 \mathrm{sec}$ & $94{ }^{\circ} \mathrm{C} \quad 30 \mathrm{sec}$ & $95^{\circ} \mathrm{C} \quad 1 \mathrm{~min}$ & $96^{\circ} \mathrm{C} \quad 30 \mathrm{sec}$ \\
\hline $58^{\circ} \mathrm{C} \quad 30 \mathrm{sec}$ & $58^{\circ} \mathrm{C} \quad 30 \mathrm{sec}$ & $50^{\circ} \mathrm{C} \quad 30 \mathrm{sec}$ & $60^{\circ} \mathrm{C} \quad 45 \mathrm{sec}$ \\
\hline $68^{\circ} \mathrm{C} \quad 40 \mathrm{sec}$ & $68^{\circ} \mathrm{C} \quad 40 \mathrm{sec}$ & $72{ }^{\circ} \mathrm{C} \quad 1 \min 30 \mathrm{sec}$ & $72{ }^{\circ} \mathrm{C} \quad 1 \mathrm{~min}$ \\
\hline 35 cycles & 36 cycles & 35 cycles & 32 cycles \\
\hline $68^{\circ} \mathrm{C} \quad 5 \mathrm{~min}$ & $68^{\circ} \mathrm{C} \quad 5 \mathrm{~min}$ & $72{ }^{\circ} \mathrm{C} \quad 3 \mathrm{~min}$ & $72{ }^{\circ} \mathrm{C} \quad 5 \mathrm{~min}$ \\
\hline $8^{\circ} \mathrm{C} \quad$ inf & $4^{\circ} \mathrm{C} \quad \inf$ & $8^{\circ} \mathrm{C} \quad$ inf & $8^{\circ} \mathrm{C} \quad$ inf \\
\hline
\end{tabular}




\subsubsection{Agarose gel electrophoresis}

$10 \mu 1$ of the PCR product was loaded on a $1.5 \%$ Agarose gel (prepared in TAE 1X buffer) containing Sybr Safe DNA gel stain (Invitrogen) for DNA labeling and run in custom-made chambers (Feinmechanik of the Max Planck Institute for Experimental Medicine, Göttingen) in TAE 1X buffer.

Detection was carried out by exposure to UV light using the Intas Gel Documentation System (Intas Science Imaging Instruments Gmbh, Göttingen, Germany).

\subsubsection{Image processing and statistical analysis}

All image processing was done using the free software Fiji-ImageJ. When calculating intensities, the imaging was done at exactly the same parameters of illumination, exposure and gain for all compared conditions. For F- and G-actin intensity quantification, the cell body was excluded.

Statistical significance was determined using GraphPad PRISM 5 software. Unless stated otherwise the statistical analysis were performed using two-tailed Student's T-test function (T-test $<0,05:^{*},<0,01: * *$ and $\left.<0,001: * * *\right)$. The variation between samples was calculated using the standard variation of the mean. For comparing 3 or more groups a one-way analysis of variance (ANOVA) was used and further pairwise using Tukey's post test. 


\section{\begin{tabular}{l|l} 
Results & 3
\end{tabular}}

This doctoral project was designed with the aim of studying the relationship between changes in the mechanical properties of a cell and its differentiation. To investigate this, it was first necessary to establish the experimental techniques to measure the mechanical properties of single cells and, in a second step, to apply these methods to the understanding of how mechanical properties control cell differentiation in oligodendrocytes. Accordingly, the results section is divided into two parts:

The first part describes the validation of a vertical optical trap as a novel tool for measuring the visco-elastic properties of cells at pico-Newton forces and will show how the mechanical response of the cell depends on the applied force, the length and directionality of deformation and the presence of the actin cortex. This work has been previously published (Nawaz*, Sánchez*, et al., 2012), *equal contribution.

The second part focuses on exploring the role of actin dynamics during myelination and oligodendrocyte differentiation. To address this, I used an interdisciplinary approach combining morphological, genetic and biophysical analyses to describe how actin polymerization and depolymerization influences myelin sheath growth in the central nervous system (CNS). This work has been accepted for publication (Nawaz*, Sánchez*, et al., 2015; *equal contribution). 
Both results sections are largely based on the two mentioned publications. To preserve the logical flow of arguments I included also some images that were obtained by co-authors. When this is the case, it is clearly mentioned in the figure caption.

\subsection{Cell visco-elasticity measured with AFM and optical trapping at sub-micrometer deformations}

\subsubsection{Response of cells to mechanical deformations}

There is a variety of techniques available for the measurement of the mechanical properties of cells, with AFM being the most commonly used to quantify cell stiffness in a liquid environment. Since the lowest force that can be reliably controlled using AFM is $\sim 0.1 \mathrm{nN}$, indenting soft cells can lead to a strain large enough to be in the nonelastic deformation regime which complicates the data analysis and increases the probability of damaging the cell. Hence, it is crucial to develop methods that enable the measurement of cells at very low forces, such as optical traps. When an optically trapped bead is used to indent the cell, the resulting deformation is expected to be comparable or lower as that in AFM indentation experiments and the same Hertzian contact model can be used to extract the Young's modulus of the cell. For this purpose we have developed an optical trap that can be operated with sub-nanometer precision in the vertical direction with respect to the cell, thus providing the same indentation geometry as in AFM. In order to validate this method, I compared the response of cells measured with this setup with similar measurements that were performed with AFM. First, I measured the response of $3 \mathrm{~T} 3$ fibroblasts to indentation at different forces using an AFM coupled to an inverted microscope and a cantilever with a $1.98 \mu \mathrm{m}$ diameter 
bead glued to its end (Figure 3.1A). Under these conditions, the applied force is distributed over a big area and potential damage to the cell is highly reduced. Next, I measured the response of the same cells at forces up to $10 \mathrm{pN}$ with our custom-built vertical optical trap. The trapped $0.76 \mu \mathrm{m}$ diameter bead was moved down to indent the cells with a piezo element moving the objective (Figure 3.1B).

A

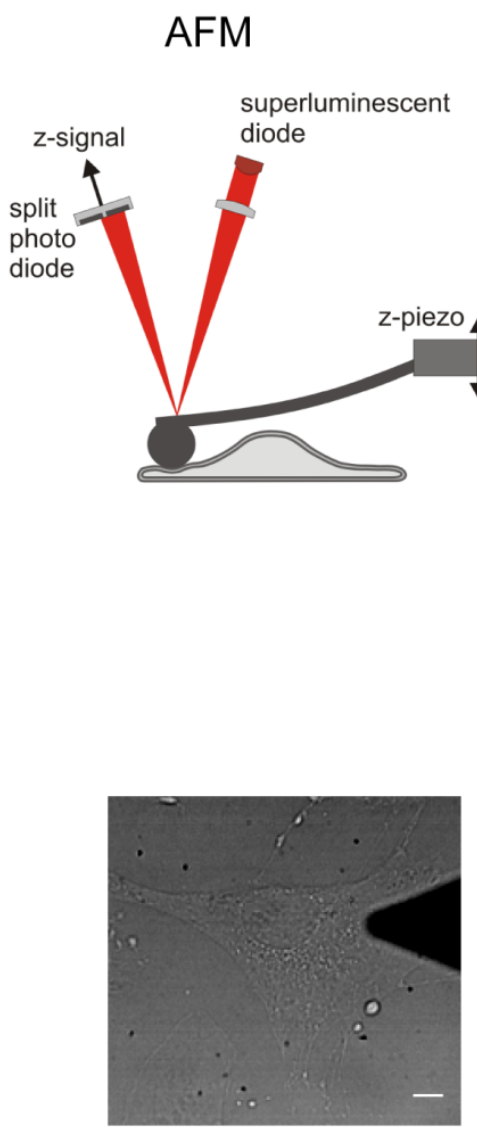

B
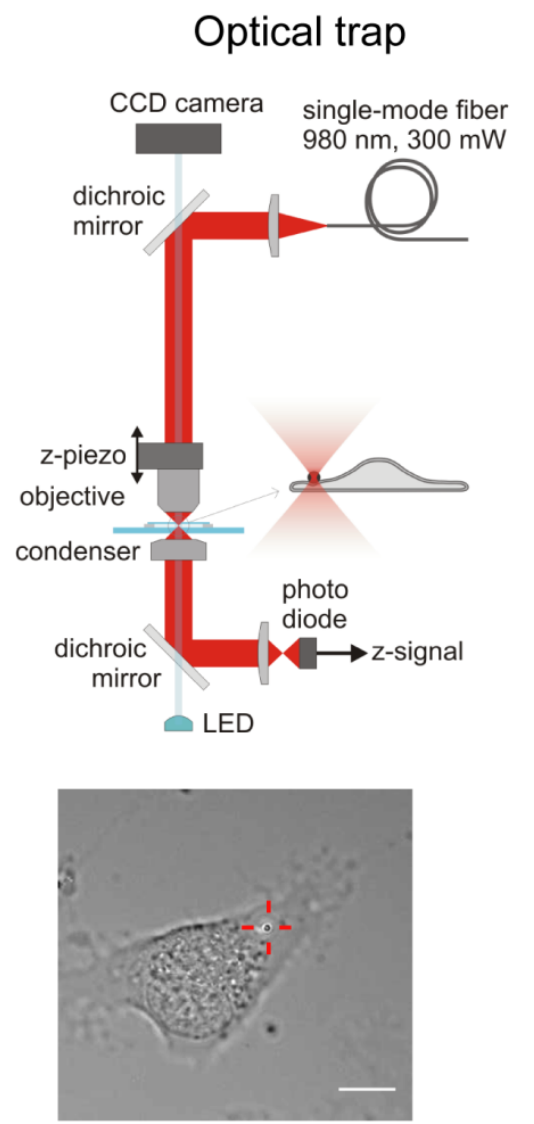

Figure 3.1: Experimental setup. (A) AFM: The cantilever is moved up and down with a z-piezo. When the AFM tip touches and indents the cell, the cantilever will bend. The amount of bending is proportional to the force applied to the cell and it is calculated using a laser beam reflected on a photodiode. The bottom image shows the tip on an AFM cantilever indenting a cell on a spot between the nucleus and the periphery. Scale bar $=10 \mu \mathrm{m}$. (B) Optical trap: A laser beam from a single mode fiber is coupled to the optical path of an upright microscope and focused onto the sample by the objective. The vertical position of the trap is controlled by a z-piezo that moves the objective up and down. The force applied to the cell is calculated by monitoring the displacement of the bead from the center of the trap using the scatter light reflected on a photodiode. The bottom image shows a cell indented by a trapped bead on a similar area as used for AFM. Scale bar $=10 \mu \mathrm{m}$. 
If the response of the cell is elastic, the indentation curve should correspond exactly to the retraction curve. Figures $3.2 \mathrm{~A}, \mathrm{~B}$ and $\mathrm{C}$ show the indentation and retraction curves performed using the AFM. Figure 3.2A shows that at an applied force of $150 \mathrm{pN}$, both curves are not identical, but show hysteresis. When indenting the cells at lower forces up to $75 \mathrm{pN}$, the hysteresis decreases (Figure 3.2B). At forces lower than $25 \mathrm{pN}$ the hysteresis cannot be distinguished anymore (Figure 3.2C) which suggests that the deformation is now mainly elastic. The high intrinsic force noise of AFM complicates data analysis and the hysteresis might be hidden in the noise. The optical trap has a much lower force noise level (Bodensiek et al., 2013), allowing a more accurate analysis (Figure 3.2D). From these optical trapping curves it is evident that there is no hysteresis at lower forces and therefore the response of cells is largely elastic as compared to using higher forces where it is not.

A

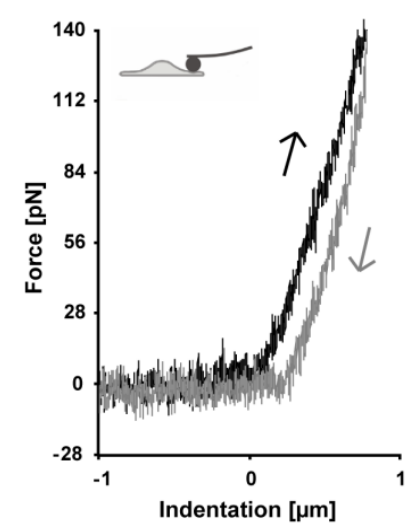

B

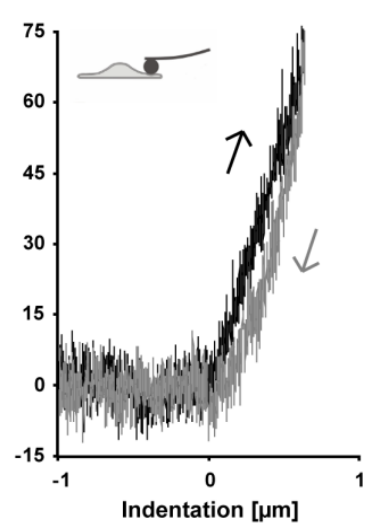

C

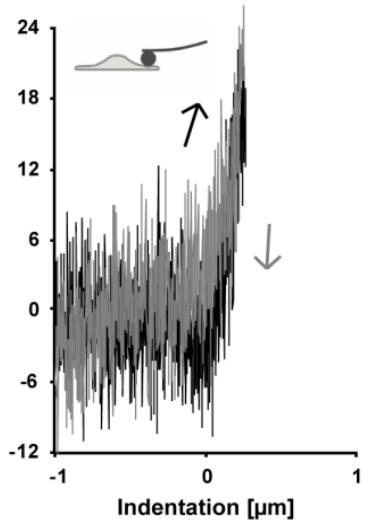

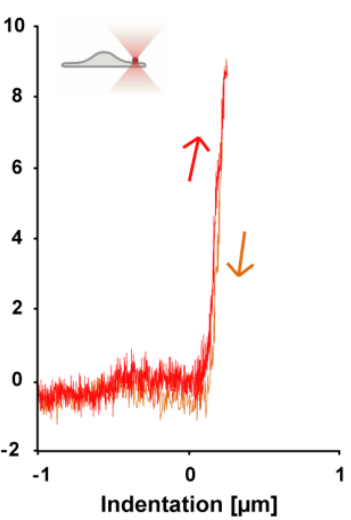

Figure 3.2: The response of cells at higher forces shows an increased hysteresis. (A) $3 \mathrm{~T} 3$ fibroblasts indented with AFM at forces around $150 \mathrm{pN}$. The indentation (black) and retraction (grey) curves are not identical, showing a clear hysteresis. (B) At a force of $75 \mathrm{pN}$ hysteresis is reduced. (C) When applying forces around $25 \mathrm{pN}$ both curves look the same and no hysteresis is visible. The intrinsic noise of AFM makes it difficult to determine the contact point and analyze the data correctly. (D) Cells indented with the optical trap at forces of around $10 \mathrm{pN}$ show no difference between indentation (red) and retraction (orange) curves. The very low noise allows for an accurate analysis of the data. 
The difference between indentation and retraction curves at higher forces indicates that some of the energy applied to indent the cell is not being recovered completely after the cell returns to its original shape. To quantify the relative amount of energy lost during this process, I calculated the difference between the integrals of indentation and retraction curves and normalized this by dividing it by the integral of the indentation curve. Figure $3.3 \mathrm{~A}$ shows that at forces below $30 \mathrm{pN}$ there is only a small amount of work lost ( $\sim 13 \%$ for optical trap and $\sim 15 \%$ using AFM). The lost work increases proportional with the force applied reaching $\sim 40 \%$ at $150 \mathrm{pN}$. At low forces the cell deformation is $\sim 250 \mathrm{~nm}$ (Figure 3.3B) and can reach up to $800 \mathrm{~nm}$ at $150 \mathrm{pN}$, suggesting that with increasing indentation of the cell, the contribution of the viscous cytoplasm on the measurements becomes more apparent. Taken together, this data shows that the response of cells is only at low forces mainly elastic and it can be reliably measured using our vertical optical trap.

A

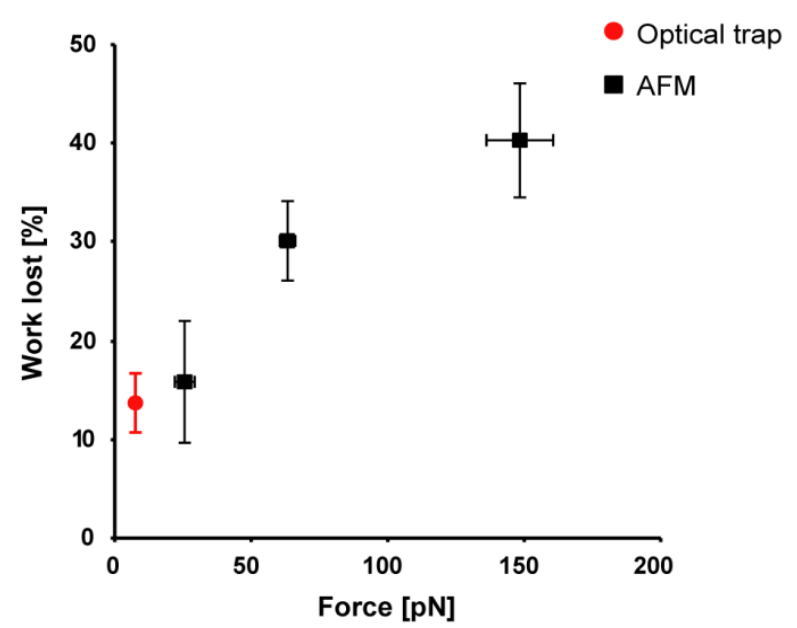

B

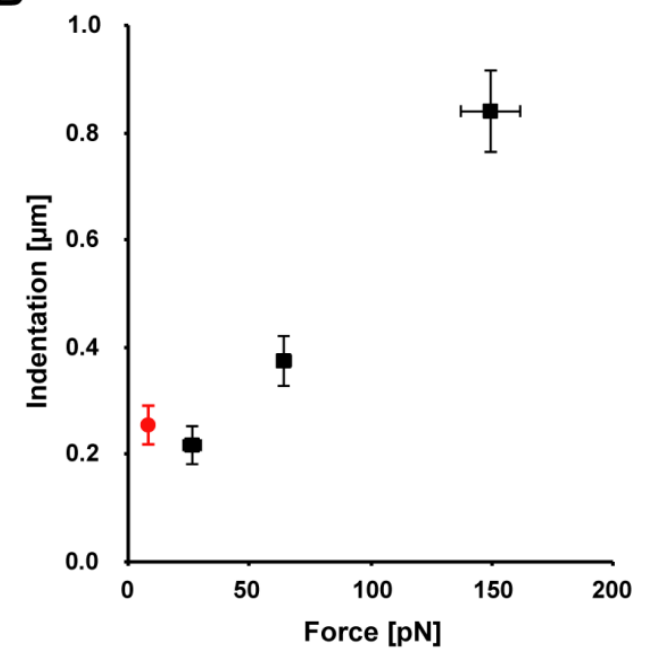

Figure 3.3: Higher deformations display a loss of energy between indentation and retraction. (A) The amount of energy lost between indentation and retraction curves was obtained by calculating the difference between the areas enclosed by each curve and dividing it by the area under the indentation curve. AFM (black) and optical trap (red) measurements at forces up to $30 \mathrm{pN}$ show an energy loss of $\sim 15 \%$. At higher forces, it increases up to $40 \%$. Graph shows mean \pm SEM ( $n=7-15$ cells). (B) Cell indentation was calculated from the estimated contact point of the curves used for (A). At forces lower 
than $30 \mathrm{pN}$ the average indentation is $0.2 \mu \mathrm{m}$ whereas at higher forces indentation can reach up to $0.8 \mu \mathrm{m}$. Graph shows mean \pm SEM ( $n=7-15$ cells $)$.

\subsubsection{The cell's response is elastic at small deformations and viscous at large deformations}

If a material is elastic, its response will be independent on the deformation rate. Therefore, I tested the elastic response of cells at indentation speeds ranging from 0.3 to $14 \mu \mathrm{m} / \mathrm{s}$ using AFM (up to $1 \mathrm{nN}$ ) and optical trap (up to $10 \mathrm{pN}$ ). Since I observed in the earlier experiments that the cellular response depends on the applied force, I analyzed the AFM indentation curves in between two ranges (Figure 3.4A), at low $(7-30 \mathrm{pN})$ and high forces $(150-600 \mathrm{pN})$. The curves were fitted using the Hertz model (Eq. 9) and the Young's modulus was calculated accordingly. As expected, small indentations using AFM and optical trap resulted in a Young's modulus that is independent from the deformation speed (Figure 3.4B, red and yellow), indicating that the response is indeed elastic. Furthermore, the values obtained with the optical trap $(103.3 \pm 10.2 \mathrm{~Pa}, \mathrm{n}=90)$ are comparable with the ones obtained using AFM $(85.3 \pm 4.5 \mathrm{~Pa}, \mathrm{n}=237)$, showing that both techniques can yield consistent results for the Young's modulus of cells. Analysis of the AFM response at high forces revealed that the Young's modulus increases from $140 \mathrm{~Pa}$ at small rates of deformation up to $330 \mathrm{~Pa}$ at higher rates (Figure 3.4B, green), confirming that the measured response is not elastic. 
A

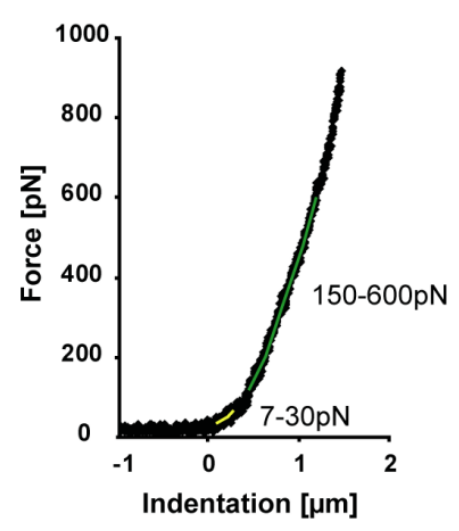

B

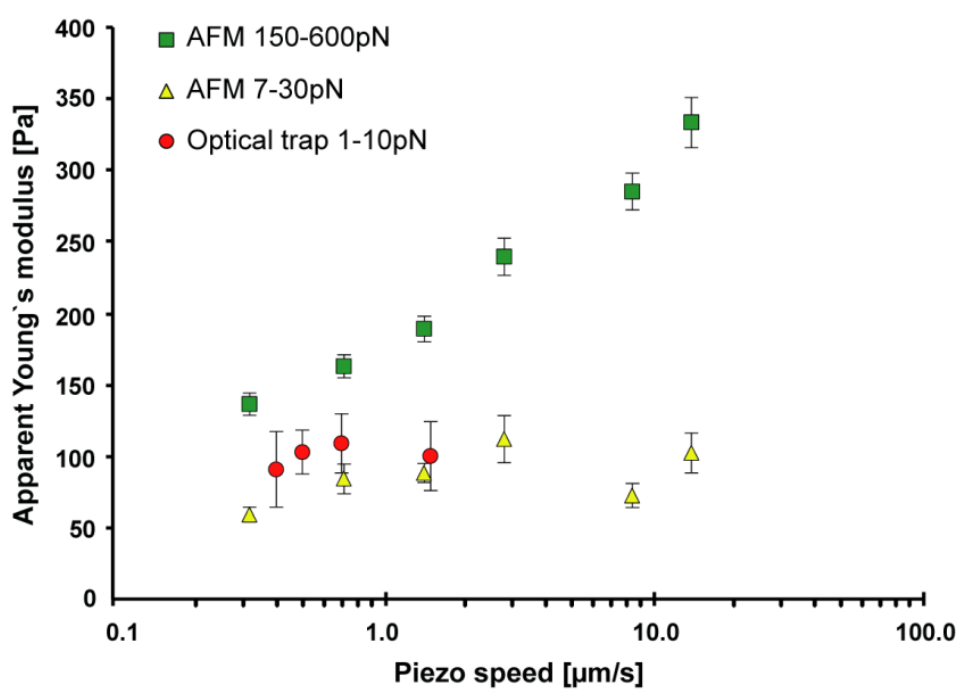

Figure 3.4: The apparent Young's modulus of the cells suggests the presence of a viscous component at higher deformations. (A) The Hertz model was used to obtain the Young's modulus of cells. AFM indentation curves were fitted between $7-30 \mathrm{pN}$ (yellow) and $150-600 \mathrm{pN}$ (green). (B) Young's modulus of optical trap (red) and AFM indentation curves fitted between $7-30 \mathrm{pN}$ (yellow), show that Young's modulus is independent of the indentation speed. In AFM indentation curves fitted between $150-600 \mathrm{pN}$ (green), the Young's modulus increased a multifold at higher indentation speeds. Graph shows mean \pm SEM ( $n=16-41$ cells per speed).

When higher forces are applied to the cell, its deformation will extend deeper into the cell, potentially increasing the influence of the viscous cytoplasm. This hypothesis is supported by the fact that the stiffness of viscous materials strongly depends on their rate of deformation, suggesting that the apparent Young's modulus measured at large deformations is most likely a combination of the elastic constant of the cell and a viscous component. However, when increasing the speed of deformation while keeping force range for analysis constant, the cell will be indented less and therefore the contribution of viscosity will be reduced. In order to correct for this potential artifact and to estimate the contribution of viscosity at a constant range of deformation $(0.15-0.45 \mu \mathrm{m}$ and 0.8-1.2 $\mu \mathrm{m})$, I plotted the apparent Young's modulus calculated for both indentation ranges as a function of its indentation rate (Figure 3.5). This reveals that the apparent modulus follows a power law with an exponent $\alpha=0.31$ at 
indentations between 0.8 and $1.2 \mu \mathrm{m}$. Because we found an elastic behavior at very small deformation, one should expect that at intermediate indentations the exponent would be decreased. This is indeed the case as observed at indentations between 0.15 and $0.45 \mu \mathrm{m}$ with an exponent $\alpha=0.17$. These results confirm that a viscous component has a significant and increasing impact on the apparent Young's modulus at larger deformations.

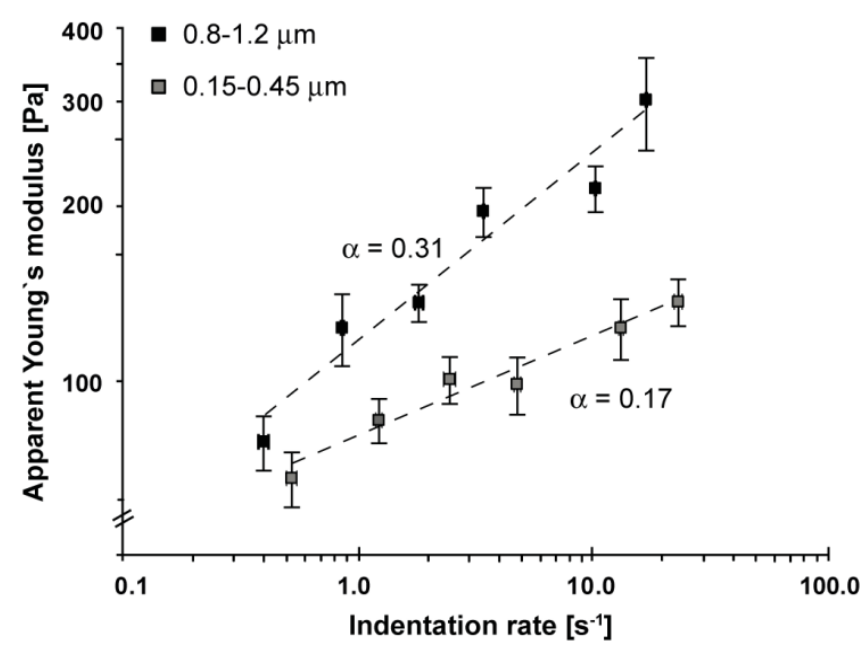

Figure 3.5: The response of the cells is viscous at high deformations and follows a power law. The apparent Young's modulus was plotted as a function of the indentation rate calculated as $f=1 /\left(2 *\left(\mathrm{t}_{2}-\mathrm{t}_{1}\right)\right)$, where $t_{2}-t_{1}$ is the time of indentation for the analyzed indentation range (plotted on a double logarithmic scale). The fit follows a weak power law. At lower indentations the exponent is 0.17 and increases to 0.31 at higher deformations. Graph shows mean $\pm \operatorname{SEM}(n=16-41$ cells $)$.

\subsubsection{The elastic response of cells at small deformations is anisotropic}

Ideally, the modulus of isotropic elastic materials is independent of the directionality of deformation. I tested this property on cells using a vertical optical trap to stretch the cells as opposed to the previous indentation experiments (Figure 3.6A). In order to calculate the Young's modulus, a variation of the Hertz model that describes the deformation of a large elastic body with a disc shaped contact area was used. In this model, the relation between the force $F$ and the deformation $d_{z}$ is linear (Eq. 10). In 
order to carry out the calculations, the contact area between bead and cell, which will be assumed to remain constant during the pulling experiment, needs to be estimated. Since it is not possible to measure this area directly, the maximum contact with the bead at the measured indentation of $255 \mathrm{~nm}$ was estimated according to (Nawaz et al., 2012), resulting in a value of $311 \mathrm{~nm}$. Next, the Young's modulus was calculated by applying a linear fit on the stretching part of the retraction curves between -10 and $0 \mathrm{pN}$ and using the estimated contact radius $\mathrm{R}_{\mathrm{c}}$. Figure 3.6B shows that the Young's modulus obtained from stretching experiments was twice as high as the calculated one from indentation experiments (239 and $100 \mathrm{~Pa}$, respectively). This demonstrates that the directionality of deformation plays a role at the time of calculating the elastic modulus of cells and confirms once more the limitation of treating the cell as an homogenous elastic material.

A

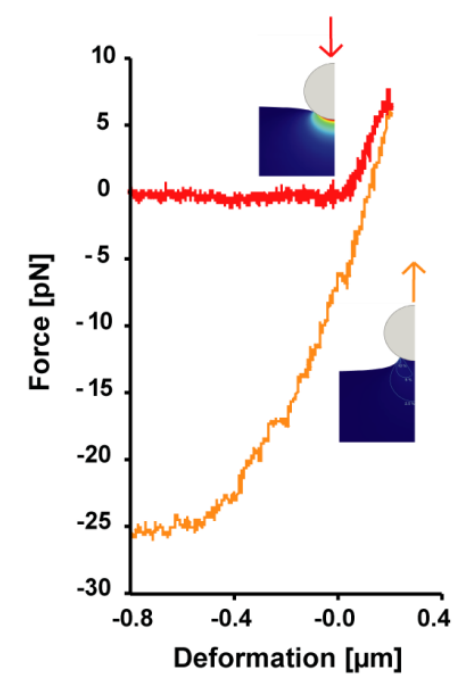

B

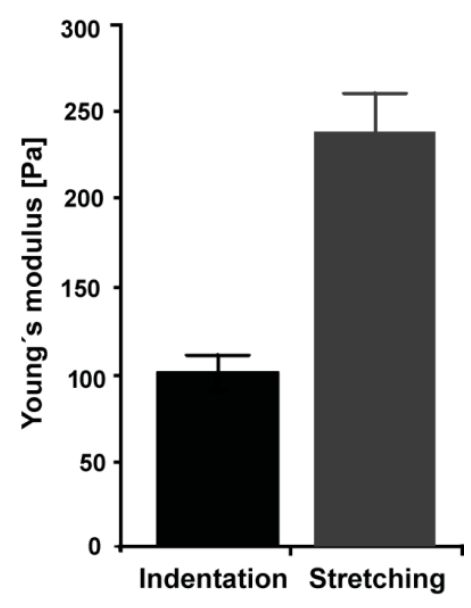

Figure 3.6: Directionality of the elastic response is anisotropic. (A) The Young's modulus of stretching experiments (orange curve) was calculated using a variation of the Hertz model. Only beads that got attached to the cell were used. (B) The estimated Young's modulus obtained from stretching experiments was twice as high $(239 \mathrm{~Pa})$ as the calculated one from indentation experiments (100 Pa). Graph shows mean \pm SEM. 


\subsubsection{The actin cortex is the major contributor to cell elasticity at small deformations}

After describing the visco-elastic response of cells at small and large deformations and showing its anisotropy, I speculated about the reason of this behavior. Using AFM at high indentations, it has been previously shown that the response of a cell to deformation depends on the actin cortex (Rotsch and Radmacher, 2000). Since our vertical optical trap setup allows cell response measurements at very small deformations, I subjected cells to indentation experiments to determine the contribution of the actin cortex. Therefore, I depolymerized actin filaments using Latrunculin-A (1 $\mu \mathrm{M}$ ) treatment for $30 \mathrm{~min}$ (Figure 3.7) and found that the Young's modulus strongly reduced from $100.3 \pm 10.2(\mathrm{n}=90)$ to $29.3 \pm 3.5(\mathrm{n}=45)$ Pa. This proves that at small deformations, the F-actin network is the main contributor to cell elasticity.

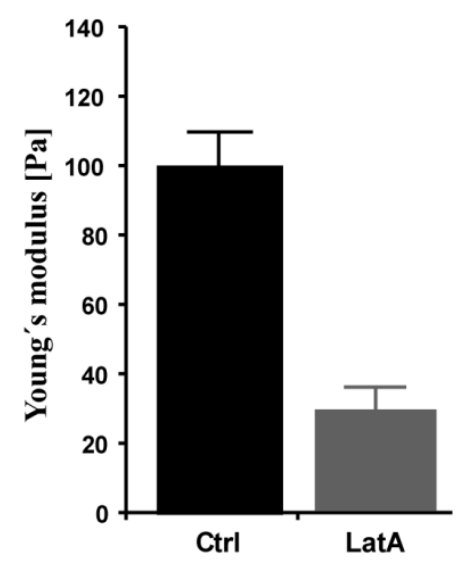

Figure 3.7: The actin cortex is the major contributor to cell elasticity at small deformations. Optical trap measurements at forces up to $10 \mathrm{pN}$ before and after F-actin depolymerization with $1 \mu \mathrm{m}$ Latrunculin-A (LatA) show a decrease of the Young's modulus. Graph shows mean \pm SEM ( $n=45-90$ cells).

Overall, these results demonstrate that the response of cells to mechanical deformation is visco-elastic, depending mainly on the depth at which the cell is indented and furthermore identifies the actin cortex as the main contributing factor for the elastic response at small deformations. 


\subsection{Actin filament turnover drives leading edge growth during myelin sheath formation in the Central Nervous System}

\subsubsection{The leading edge of oligodendrocytes shows an adhesion independent model of protrusion}

Myelin sheath extension in the central nervous system is the result of leading edge growth of an oligodendrocyte process between the innermost myelin layer and the axon followed by a lateral extension of all myelin layers along the axon (Snaidero et al., 2014). This model implies that forces need to be generated at the leading edge in order to allow the membrane to be continuously displaced. Typically, such forces are generated by F-actin polymerization, e.g. at the cell's front to push out membrane protrusions and hence we hypothesized a role of actin in the forward movement of the leading edge.

As a prerequisite for this hypothesis, F-actin should be localized at the leading edge during the period of myelination. To asses this, mouse spinal cord sections at the onset of myelination ( $\mathrm{P} 4,4$ days postnatal), when the wrapping process is taking place, were stained and compared with animals at P12, when myelin growth is being finished. MBP and F-actin staining revealed that at $\mathrm{P} 4$, actin is confined to the inner tongue of the growing myelin sheath (Figure 3.8A) disappearing almost completely from it by P12, suggesting that actin could indeed be the driving force in myelin wrapping. Since it is technically difficult to visualize the inner tongue in vivo, primary cultures of oligodendrocytes are a valuable tool to investigate sheet formation and differentiation in 
vitro. Figure $3.8 \mathrm{~B}$ shows that F-actin is distributed in the processes and the lamellipodia-like protrusions in immature oligodendrocytes. Once the cells start to form sheets, F-actin is confined to the outer rim in a structure similar to the lamellipodium of migrating cells and analogous to the leading edge of myelin sheets in vivo. At later differentiation stages, F-actin is completely depleted from the sheets, demonstrating a redistribution of F-actin during oligodendrocyte differentiation.

A

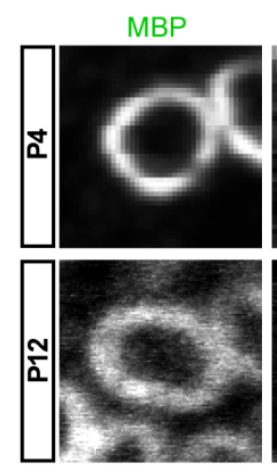

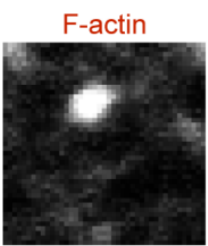

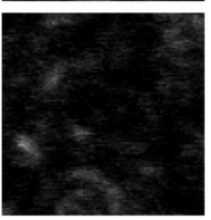

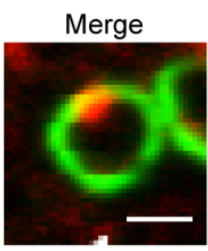

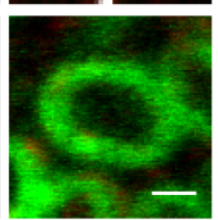

B
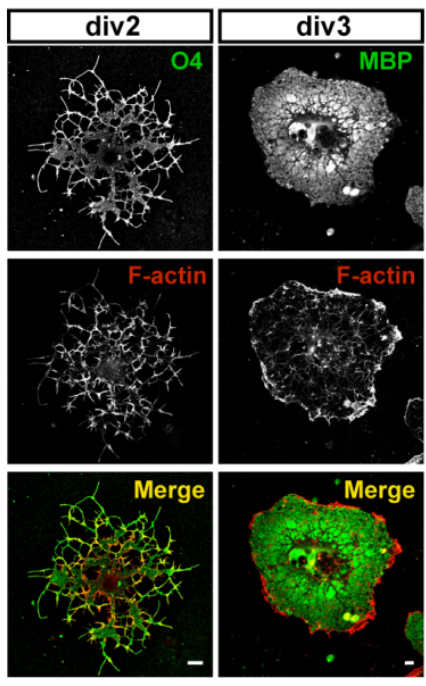
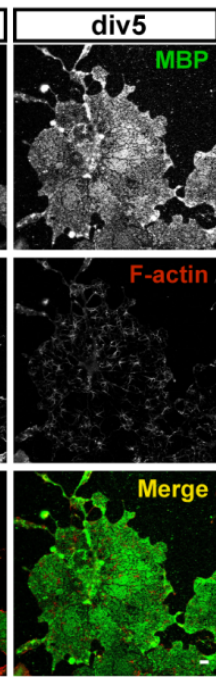

Figure 3.8: F-actin is redistributed during oligodendrocyte differentiation. (A) F-actin and MBP localization in $400 \mu \mathrm{m}$ thick spinal cord sections of WT mice show that upon termination of myelin growth actin is no longer localized at the leading edge. Scale bar $=1 \mu \mathrm{m}$. [Images kindly provided by Nicolas Snaidero] (B) Mouse primary oligodendrocytes cultured for $2-5$ days in vitro (div) display a similar redistribution of F-actin after MBP containing sheets are formed. Scale bar $=10 \mu \mathrm{m}$. [Images kindly provided by Nicolas Snaidero and Schanila Nawaz].

Since the lamellipodium is the initiation site of motility and adhesion, I used Interference Reflection Microscopy (IRM) to visualize membrane dynamics and adhesion during myelin sheet growth in oligodendrocytes. This method is very useful for the study of cells that are in close contact with the substrate (Barr and Bunnell, 2009; Verschueren, 1985) and does not require labeling. Due to destructive interference of the reflected light from the coverglass, which depends on the distance between the 
cell membrane and the glass surface, an image displaying dark pixels at areas where the membrane is in close proximity with the surface and bright pixels representing nonadhesive areas is obtained. Using this method, I acquired time-lapse videos of oligodendrocytes grown on Poly-L-Lysine for $6-8$ hours when the cells are still in the oligodendrocyte precursor (OPC) stage and are starting to form processes, compared to 4-day-old oligodendrocytes (OLG), which are forming sheets (Figure 3.9). At a very early stage, oligodendrocyte precursor cells (OPC) extend processes with a growth cone-like structure at its end. This structure shows mainly destructive interference indicating that it is adhered to the surface (Figure 3.9, OPC). At later stages however, when the cells are only forming sheets, the leading edge is highly dynamic and less adherent to the surface, as indicated by the increase of bright pixels (Figure 3.9, OLG). These results suggest that the leading edge of oligodendrocytes is changing its adhesive properties when the cell is extending processes as compared to when it is forming membrane sheets.
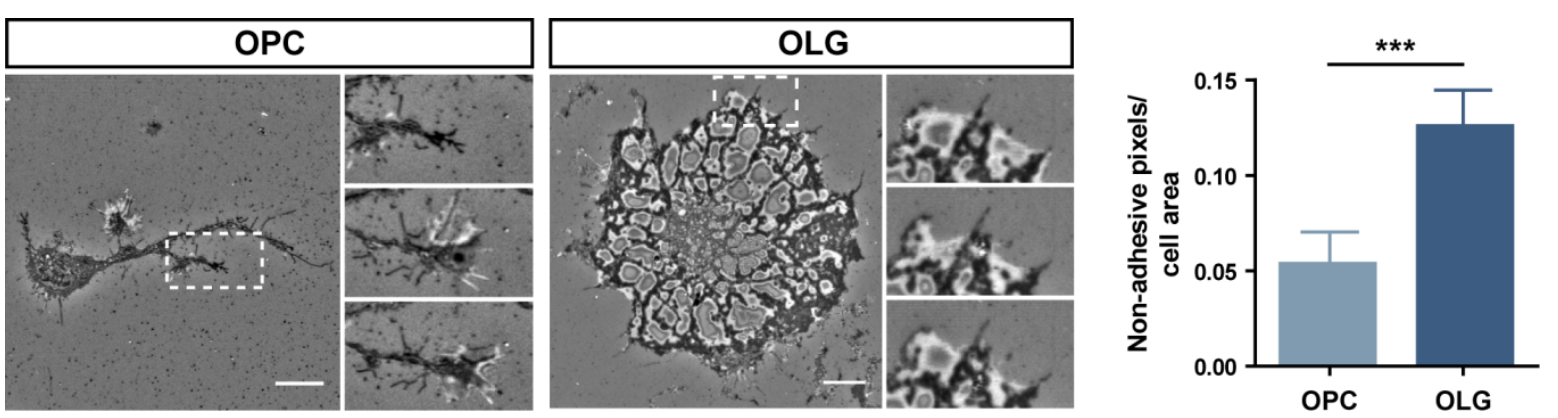

Figure 3.9: The leading edge of oligodendrocyte precursors is more adhesive than the leading edge of sheet forming oligodendrocyte. Live imaging of primary oligodendrocyte precursor cells (OPC) and sheet forming oligodendrocytes (OLG) using Interference Reflection Microscopy (IRM) to visualize membrane dynamics. The inset shows an enlarged area of the leading edge of each cell over time. Quantification shows more non-adhesive areas in sheet forming as compared to precursor cells. Graph shows mean $\pm \operatorname{SD}(n=9-15$ cells from each stage, $* * * p<0.001, t$-test $)$. Scale bars $=10 \mu \mathrm{m}$.

Since the leading edge displays different degrees of adherence depending on the differentiation stage of the cell, I tested whether the adhesive contact with the surface is 
required for its stability by treating the cells with $200 \mu \mathrm{g} / \mathrm{ml}$ of Trypsin to disrupt the adhesive contacts (Figure 3.10). As expected, treatment only affected OPCs, in which the leading edge completely retracted after treatment and therefore showed a decrease in the cell projected area. In contrast, treatment had no effect on the leading edge as well as the cell projected area of sheet forming cells (OLG).
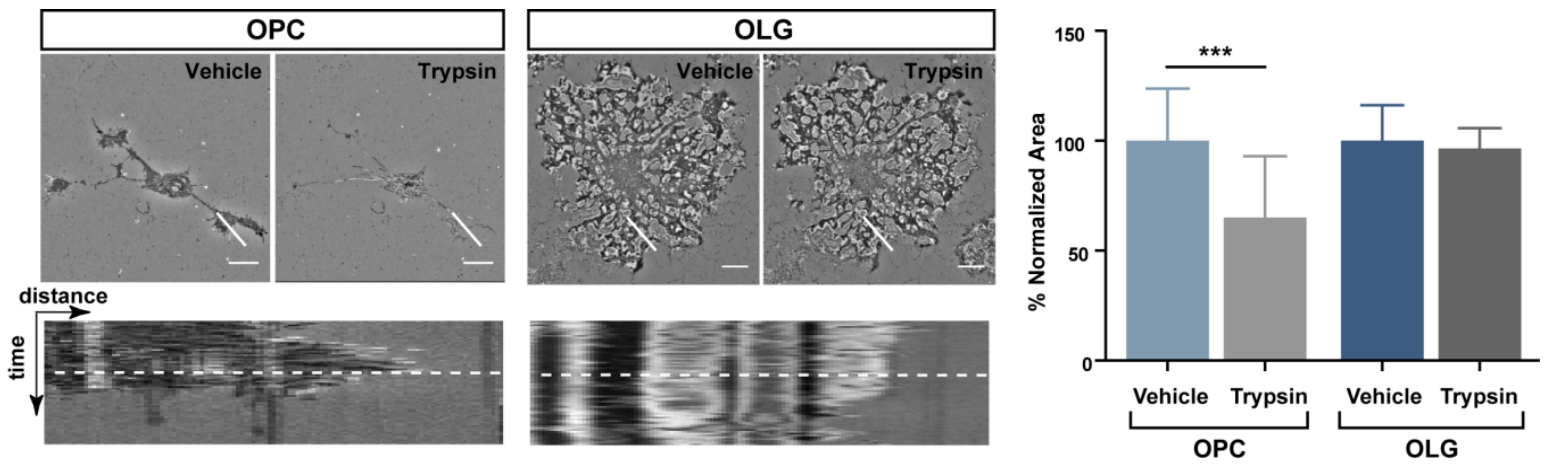

Figure 3.10: Disruption of the cell-substrate interactions collapses the leading edge of precursor but not sheet forming oligodendrocytes. IRM images of precursor (OPC) and sheet forming cells (OLG) before and after treatment with $200 \mu \mathrm{g} / \mathrm{ml}$ Trypsin. Kymographs (lower panel) show the movement during $30 \mathrm{~min}$ of the area shown by the white lines; dotted lines mark the addition of Trypsin. Quantification shows a decrease in normalized cell projected area only for OPCs after treatment. Graph shows mean $\pm \mathrm{SD}\left(\mathrm{n}=25-38\right.$ cells, ${ }^{* * *} \mathrm{p}<0.001$, t-test $)$. Scale bars $=10 \mu \mathrm{m}$.

The aforementioned results suggest that cells form adhesive contacts only at early differentiation stages. To confirm this, I stained oligodendrocytes (OPCs and OLGs) as well as astrocytes plated on Poly-L-Lysine-coated coverglasses against Vinculin, a protein located in focal adhesion complexes (Figure 3.11A). As expected, I could only visualize Vinculin-postive staining on processes of OPCs, which is located at the base of the growth cone structure. OLGs however, did not display Vinculin-positive staining on their sheets. Since adhesive contacts are formed specifically between proteins of the Extracellular matrix (ECM) and the cell, I cultured cells on $100 \mu \mathrm{g} / \mathrm{ml}$ PLL with $100 \mu \mathrm{g} / \mathrm{ml}$ Fibronectin. Comparable to PLL coating, astrocytes formed focal adhesions clearly visible by Vinculin-positive staining (Figure 3.11B). In 
oligodendrocytes however, no formation of adhesive contacts was found as shown by the highly significant lower mean intensity of Vinculin staining when compared to astrocytes (Figure 3.11B).

A

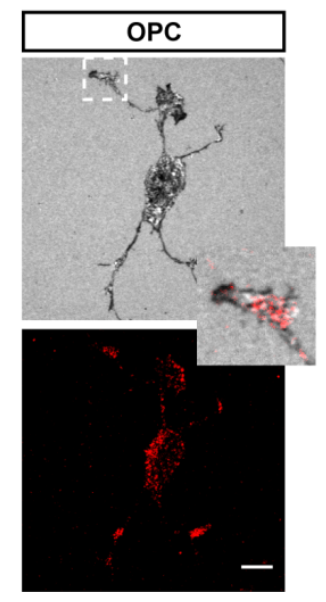

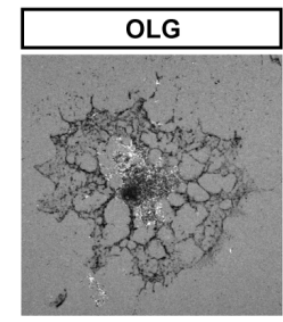

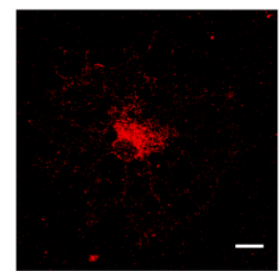

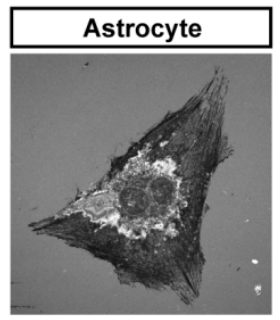

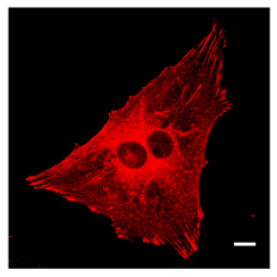

B

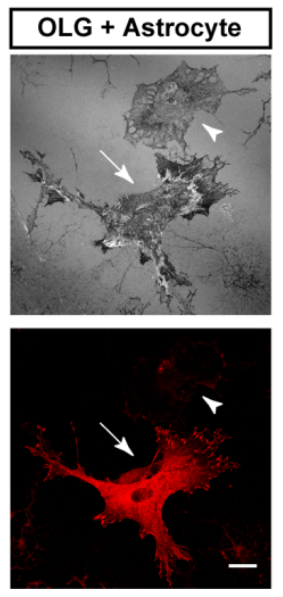

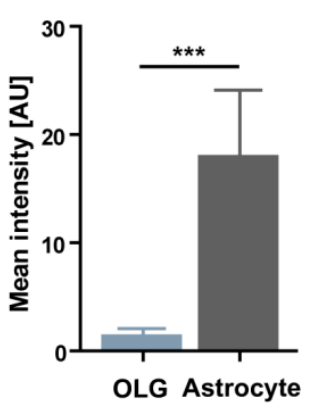

Figure 3.11: Vinculin is only present it early stage OPCs. (A) Primary oligodendrocytes plated on 100 $\mu \mathrm{g} / \mathrm{ml}$ Poly-L-Lysine coated coverslips express Vinculin (red) only at early stages of differentiation (OPC) but not at later stages (OLG). Inset shows an enlarged view of the leading edge with Vinculin located at its base. The right most panel shows an astrocyte as comparison. Scale bars $=10 \mu \mathrm{m}$. (B) Shows an oligodendrocyte (arrow head) and an astrocyte (arrow) cultured on $100 \mu \mathrm{g} / \mathrm{ml}$ Poly-L-Lysine with $100 \mu \mathrm{g} / \mathrm{ml}$ Fibronectin coated coverslips for 4 days. Quantification shows that the mean fluorescence intensity of oligodendrocytes is much lower than astrocytes. Graph shows mean \pm SD $(n=14$ cells, $* * * p<0.001$, t-test). Scale bar $=10 \mu \mathrm{m}$.

Taken together, these results demonstrate that the growth cone-like structures of oligodendrocyte processes require adhesive contacts for their assembly and moreover that when oligodendrocytes terminate their process outgrowth phase, the leading edge becomes non-adhesive until sheet formation is complete. 
Membrane motility ceases and adhesive contacts are formed after actin depolymerization

IRM time-lapse images revealed that oligodendrocytes have highly motile areas during process extension and sheet formation, but this motility decreased after sheet formation is completed (Figure 3.12A). Inversely, adhesive areas start to increase reaching the maximum when sheet formation is complete (Figure 3.13A). Moreover, upon transduction of cells with Lifeact-GFP to visualize actin dynamics, non-adhesive and highly motile areas display enrichment in F-actin (data not shown).

A
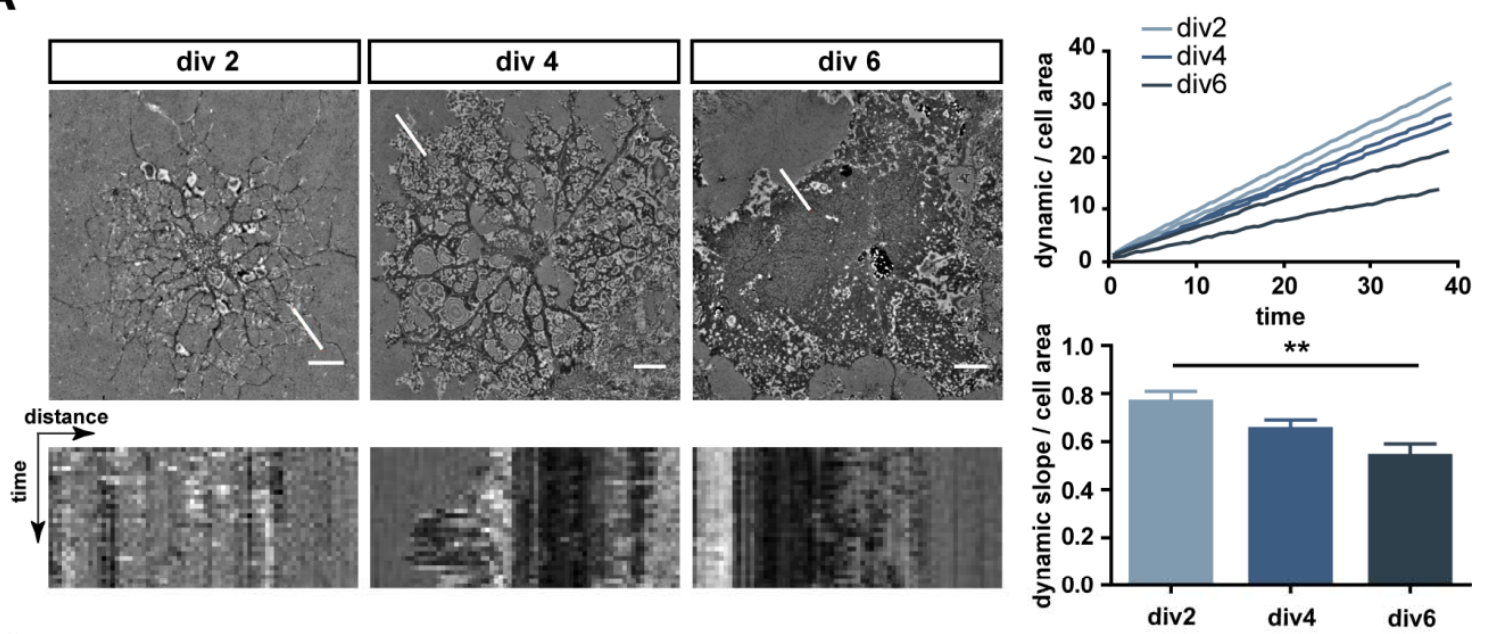

B
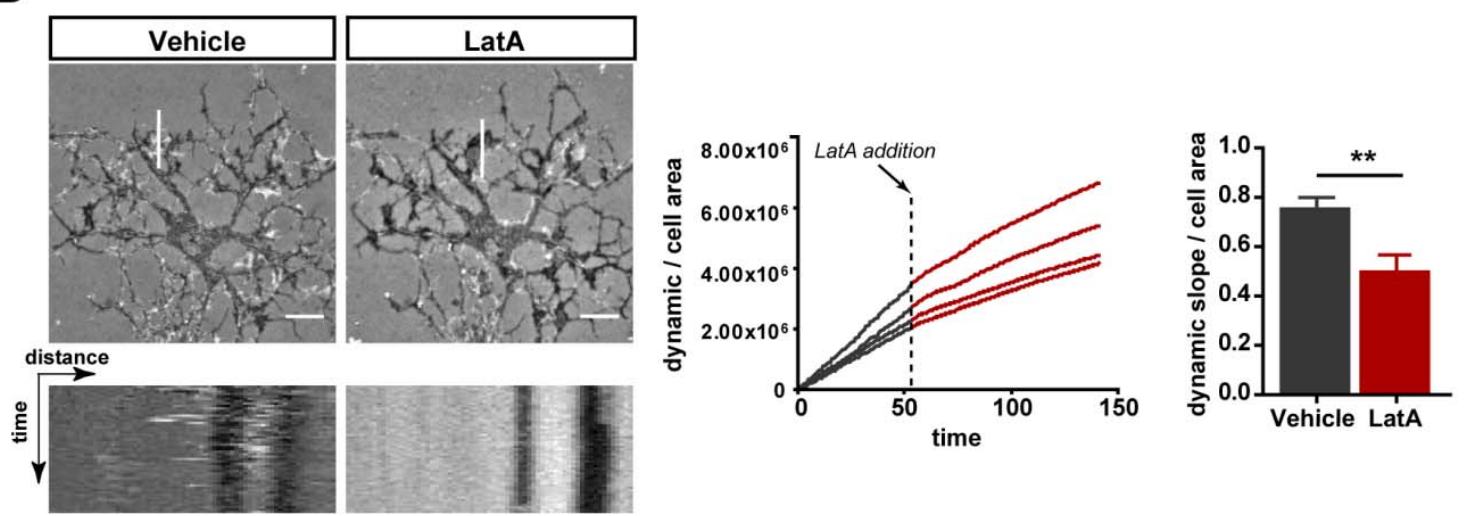

Figure 3.12: Membrane motility decreases after actin depolymerization. (A) IRM images were used to quantify membrane dynamics of primary oligodendrocytes cultured for $2-6$ days. Kymographs (lower panel) show a dynamic membrane that remains static after differentiation. The upper graph shows dynamic pixels from single cells at different differentiation stages over time. Quantification in the bottom graph shows a decrease of dynamic areas (normalized by the total cell area) upon differentiation. Graph 
shows mean $\pm \operatorname{SEM}(\mathrm{n}=9-21$ cells for each stage, $* * \mathrm{p}<0.01$, $\mathrm{t}$-test $)$. Scale bars are $=10 \mu \mathrm{m}$. [Data acquisition by Schanila Nawaz] (B) IRM images before and after F-actin depolymerization with Latrunculin-A (LatA). Kymographs (lower panel) demonstrate that motility stops. The graph on the left shows dynamic pixels from four different cells with the dotted line marking LatA addition. Quantification on the right-hand graph shows a decrease of dynamic areas (normalized by total cell area) upon treatment. Graph shows mean $\pm \operatorname{SEM}(\mathrm{n}=9$ cells, $* * \mathrm{p}<0.01$, paired t-test). Scale bars $=10 \mu \mathrm{m}$. [Data acquisition by Schanila Nawaz].

A

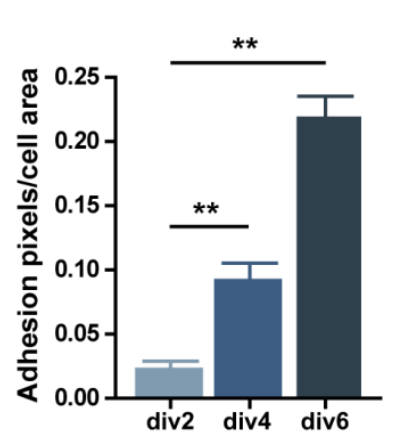

B

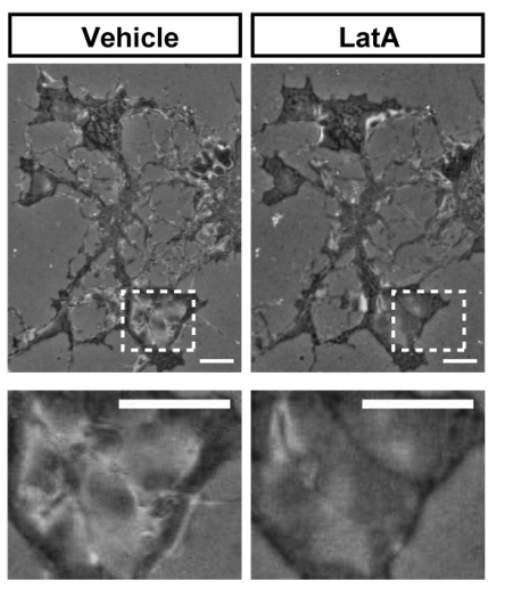

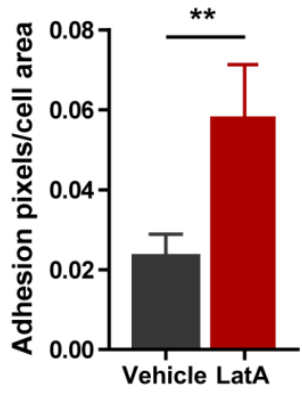

Figure 3.13: Adhesive areas increase upon actin depolymerization. (A) Quantification of adhesive areas normalized by total cell area of the cells from figure 3.12A shows an increase upon differentiation. Graph shows mean \pm SEM ( $\mathrm{n}=9-21$ cells for each stage, ${ }^{* *} \mathrm{p}<0.01$, t-test). (B) IRM images of oligodendrocytes before and after F-actin depolymerization with Latrunculin-A (LatA). Quantification shows an increase of adhesive areas after treatment. Graph shows mean \pm SEM $\left(n=9\right.$ cells, ${ }^{* *} p<0.01$, paired t-test). Scale bars $=10 \mu \mathrm{m}$. [Data acquisition by Schanila Nawaz].

Consequently, it is conceivable that oligodendrocytes, similarly to non-adhesive cells such as leukocytes, can generate the friction they require to push the leading edge forward by clamping it between two surfaces in a 3D environment. In this model, they would use F-actin polymerization to "inflate" the leading edge in between the axon and the newly formed myelin sheath. Therefore, a depolymerization of F-actin should trigger the collapse and subsequent spreading of the protrusions when the cell is on a 2D substratum. To test this, cells that had formed thin processes and were starting to form sheets were treated with Latrunculin-A (LatA) to depolymerize F-actin. I could observe 
that motility ceased immediately (Figure 3.12B) and adhesion to the surface was triggered (Figure 3.13B). When the amount of membrane spreading was quantified, an increase of the cell projected area of LatA treated cells was found (Figure 3.14). This response is the opposite of what would happen if F-actin was depolymerized on a cell with a linkage to the surface mediated by adhesion molecules, supporting the hypothesis of an adhesion-independent and deformation-based model of leading edge protrusion.

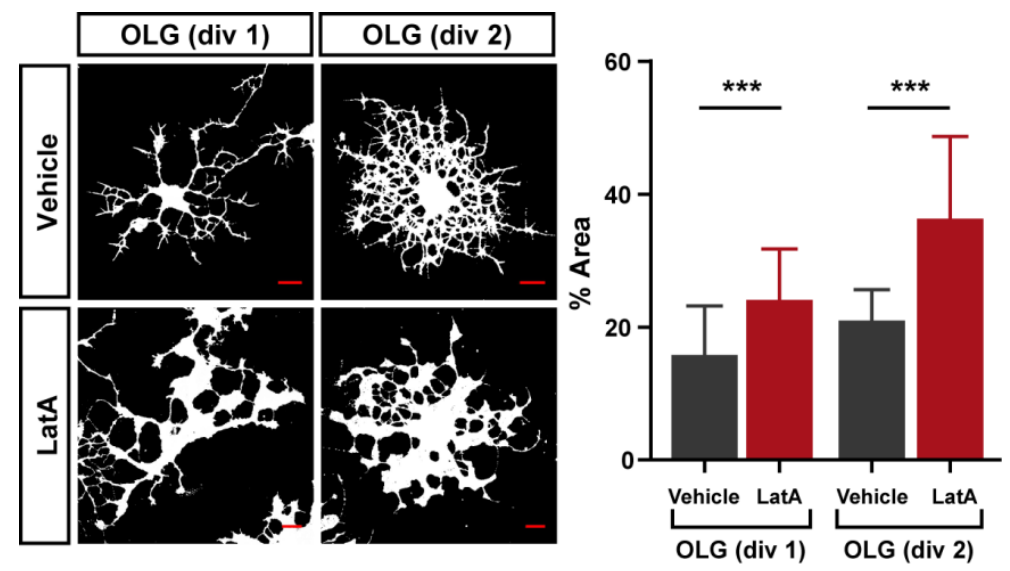

Figure 3.14: F-actin depolymerization triggers membrane spreading. Oligodendrocytes cultured for 1 or 2 days, treated with $10 \mu \mathrm{M}$ Latrunculin-A (LatA) for 4 hours and labeled with Cell Mask Orange. Images are shown in binary format. Quantification shows an increase in cell surface projected area after treatment. Graph shows mean $\pm \mathrm{SD}\left(\mathrm{n}=18-32\right.$ cells, ${ }^{* * *} \mathrm{p}<0.001$, t-test $)$. Scale bars $=10 \mu \mathrm{m}$. [Figure kindly provided by Shanila Nawaz].

To confirm that actin depolymerization is indeed the driving force for membrane spreading, I treated oligodendrocytes with Jasplakinolide (Jaspl), an F-actin stabilizing agent, and recorded IRM videos until 30 minutes after treatment. Similarly to the behavior observed for actin depolymerization with LatA, dynamics significantly decreased after treatment (Figure 3.15). However, no membrane spreading was triggered. On the contrary, there was a slight decrease of cell projected area and adhesion (Figure 3.15). These results agree with the model formerly proposed and show that only actin depolymerization is able to release the membrane and generate surface spreading and adhesion. 

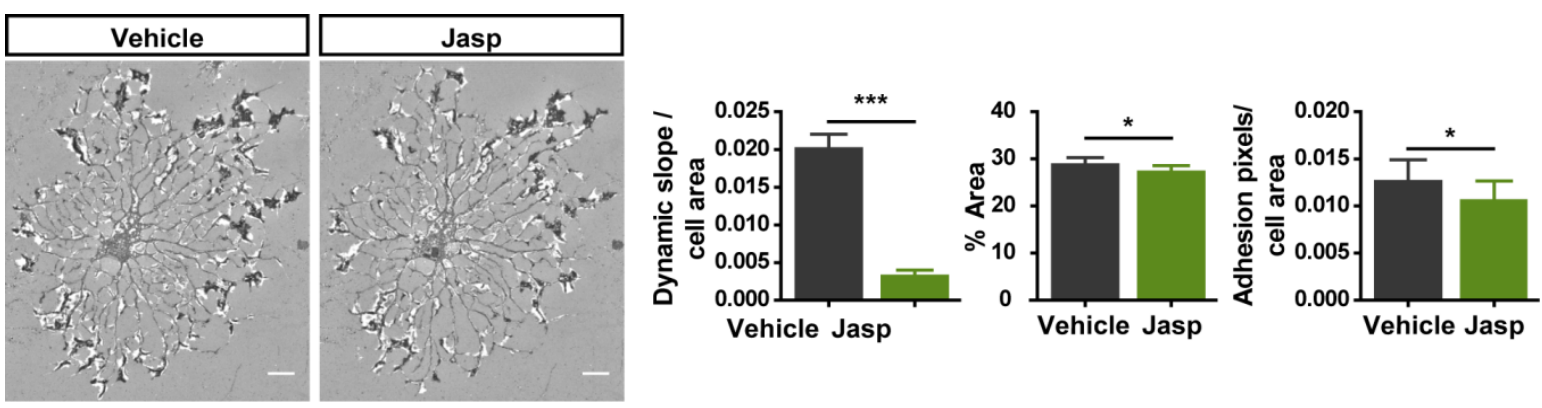

Figure 3.15: F-actin stabilization decreases motility but does not trigger membrane spreading. IRM images before and after F-actin stabilization with $200 \mathrm{nM}$ Jasplakinolide (Jasp). Quantification shows a decrease of dynamic areas, adhesive pixels and cell projected area upon treatment. Graph shows mean \pm SEM ( $\mathrm{n}=24$ cells, ${ }^{*} \mathrm{p}<0.05,{ }^{* * *} \mathrm{p}<0.001$, paired t-test). Scale bars $=10 \mu \mathrm{m}$

\subsubsection{Oligodendrocytes build up a membrane reservoir during differentiation}

Since I observed a striking spreading of membrane from very thin processes, it was logical to ask where the excess of membrane is originating from. One possibility is membrane addition through exocytosis and/or block of endocytosis. However, this possibility was ruled out using capacitance measurements by whole-cell patch clamp which measure the total amount of membrane. This was unaffected by LatA treatment (Figure 3.16A), suggesting that the excess of membrane is already present in the protrusions as folds.

A

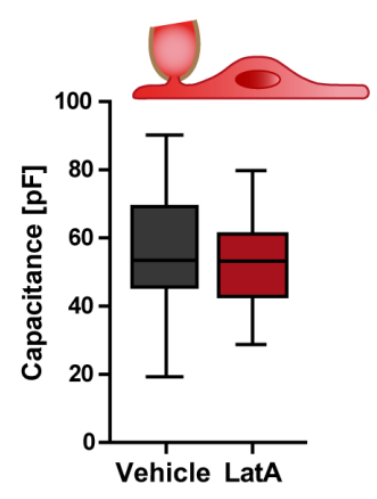

B

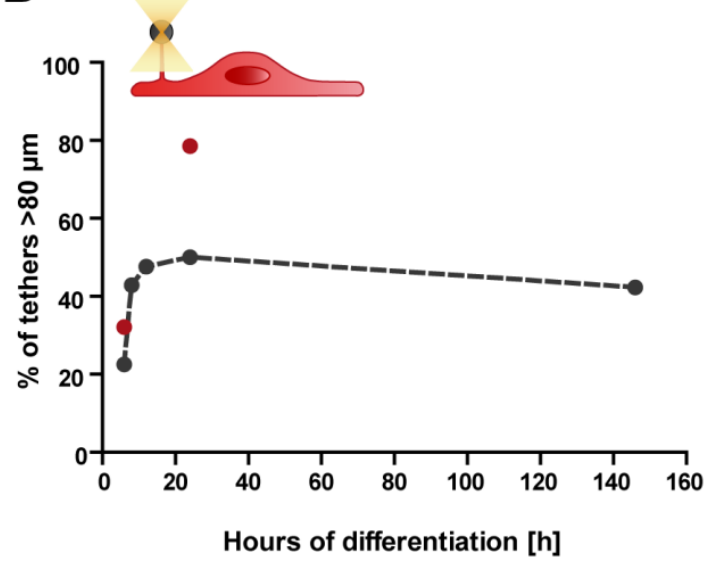

C

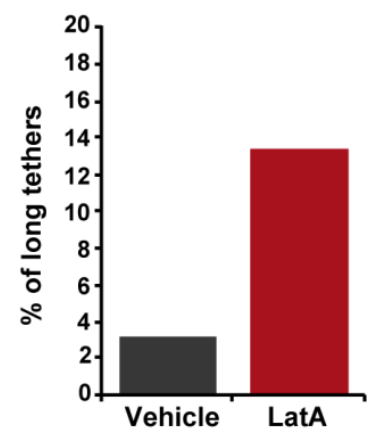

Figure 3.16: Oligodendrocytes build up a large membrane reservoir during differentiation. (A) Whole cell patch clamp capacitance measurements were performed to quantify changes in cell surface 
area. Quantification shows that (LatA) treatment for 2 hours had no effect on cellular capacitance; ( $\mathrm{n}>30$ cells). [Figure kindly provided by Schanila Nawaz] (B) Excess membrane area was quantified using an optical trap setup. Percentage of tethers that exceeded the maximal extension of the setup $(80 \mu \mathrm{m})$ was determined at different times after plating of oligodendrocytes $(6,8,12,24,146$ hours) (grey dots) showing an increase during differentiation. After $2 \mathrm{~h}$ of Latrunculin-A treatment (LatA) (red dots) there is a larger increase of membrane excess area available to be released on cells after 24 hours compared with the cells after 6 hours of plating. (C) Control experiment on NIH3T3 fibroblasts shows that upon F-actin depolymerization the amount of membrane available increases, but does not reach levels similar to oligodendrocytes.

These membrane stores can be measured by using an optical trap to pull small tubes of membrane, called tethers. The more membrane is available, the longer the tethers should be that can be pulled by this method (Raucher and Sheetz, 1999). To test this, I used the vertical optical trap setup to pull the membrane from oligodendrocytes after various times of differentiation and found that after 6 hours, at which cells are just reaching the phase of process formation, a fraction of around $20 \%$ of tethers longer than the limit of our setup $(80 \mu \mathrm{m})$ could be pulled (Figure 3.16B, grey dots). This number increased rapidly to almost $50 \%$ after only 12 hours of plating, suggesting that the cell has accumulated a membrane reservoir as infoldings. In this line, I reasoned that the excess of membrane might be starting to accumulate in the processes from early stages of differentiation but it is kept together by the actin cytoskeleton. If this is the case, then depolymerizing F-actin should release this membrane reservoir. After 6 hours of plating, when the membrane reservoir still seems to be small, only a small amount of membrane is released after LatA treatment (Figure 3.16B, red dots). On the contrary, after 24 hours of plating when the cell is starting to form sheets, the amount of membrane released is much higher in comparison. To confirm that the release is due to an increase of membrane reservoir and not just a result F-actin depolymerization, I tested NIH3T3 fibroblasts, which are known to have a smaller reservoir and observed that untreated cells have indeed less excess of membrane compared the earliest time 
point of oligodendrocyte differentiation (Figure 3.16C). As expected, there is a release of membrane infoldings after LatA treatment, which, however, is substantially lower than in the oligodendrocytes. This confirmed the idea of oligodendrocytes accumulating membrane in form of reservoirs at early stages of differentiation in order to use it later, after actin depolymerization, to expand membrane sheets.

\subsubsection{F-actin depolymerization decreases surface tension}

The excess of membrane does not fully explain the membrane spreading after actin depolymerization. Normally, when F-actin depolymerizes, the cell will round up, since the high surface tension and the forces generated by the actin cortex to keep the membrane anchored to the surface are gone. This is not the case for oligodendrocytes, since their cells surface spreads instead of rounding up. In order for this to happen, the forces keeping the membrane adhered to the surface should be higher than the surface tension pulling it up. We therefore measured surface tension by pulling tethers with the vertical optical trap. The force required to pull these tubes is determined by the interaction of membrane with the actin cortex and the membrane bending rigidity (Sheetz and Dai, 1996). I found that the static tether force of oligodendrocytes in their process forming phase is lower $(\sim 11 \mathrm{pN})$ as compared to fibroblasts $\left(\sim 13 \mathrm{pN},{ }^{*} \mathrm{p}<0.05\right.$, t-test) and further lowers when they mature sheet-forming oligodendrocytes $(\sim 6 \mathrm{pN})$ (Figure 3.17A). This shows that membrane tension decreases upon myelin formation. Furthermore, it is possible to reduce the membrane tension of process forming cells by depolymerizing F-actin agents to values similar to those of mature cells. This is specific for F-actin depolymerization since inhibitors of actomyosin contractility had no effect on surface tension. Taken together, these results demonstrate a reduction of surface 
tension during myelin sheet formation that it is completely dependent on the presence or absence of F-actin.

A
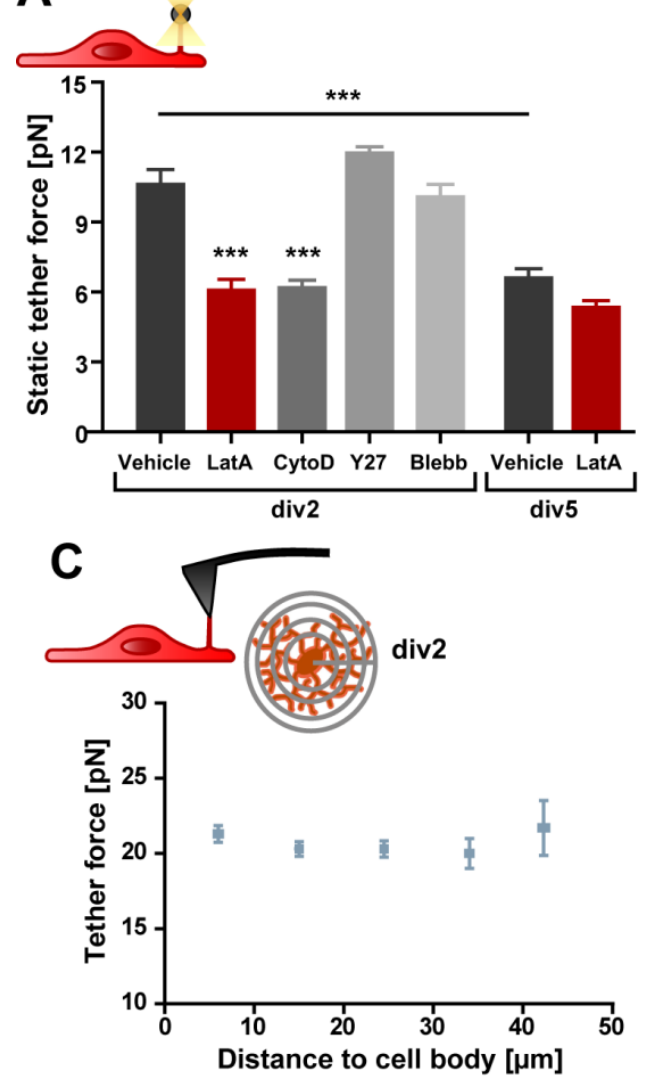

B
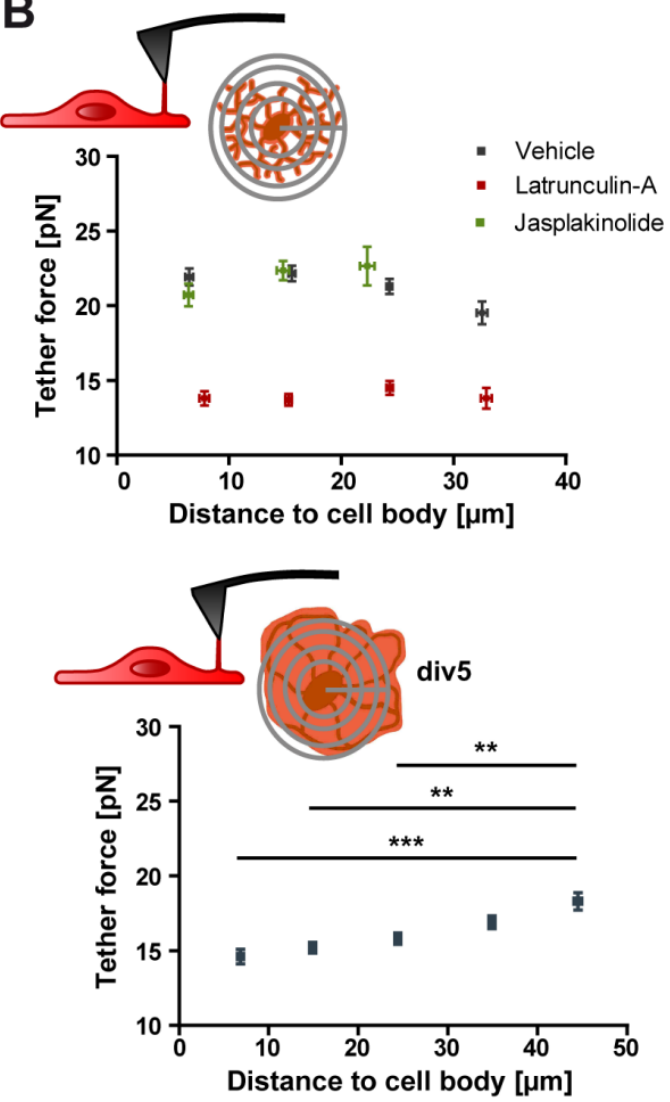

Figure 3.17: Depolymerization of F-actin reduces membrane tension. (A) Surface tension of oligodendrocytes cultured for 2 and 5 days was measured using an optical trap to pull membrane tethers. Static tether force decreases with differentiation as well as by depolymerizing F-actin for $2 \mathrm{~h}$ with $5 \mu \mathrm{M}$ Latrunculin-A (LatA) or $5 \mu \mathrm{M}$ Cytochalasin-D (CytoD). $50 \mu \mathrm{M}$ blebbistatin (Blebb) and $10 \mu \mathrm{M}$ Y27631 (Y27) had no effect. Graph shows mean \pm SEM ( $n=37-80$ cells for each condition, $* * * p<0.001)$. [Data acquisition by Schanila Nawaz] (B) Force maps of oligodendrocytes cultured for 2 days were obtained using Atomic Force Microscopy (AFM) to pull tethers. Only after LatA treatments could a decrease in tether force along the cell could be observed. Graph shows mean \pm SEM $(n=10-13$ cells with a total of $67-245$ pulled tethers per treatment). (C) Force maps of oligodendrocytes cultured for 5 days show a decrease in tether force at most parts of the cell except for the outer rim as compared to div2 cells where the tether force is homogeneous along the whole cell area. Graphs show mean \pm SEM $(n=9-12$ cells with a total of $291-800$ pulled tethers for each stage, ${ }^{* *} \mathrm{p}<0.01,{ }^{* * *}<<0.001$, one-way ANOVA and Tukey's multiple comparison test).

Since this data indicates that oligodendrocytes use actin polymerization forces located at the leading edge, I measured surface tension at different positions along the 
cell using AFM, allowing a better spatial resolution for surface tension measurements at the leading edge. To confirm that this method is applicable in this context, I treated immature oligodendrocytes with LatA and Jasplakinolide and, first of all, confirm that the tether force decreases all over the cell area after F-actin depolymerization (Figure 3.17B). Stabilization of F-actin by Jasplakinolide on the other hand had no effect on surface tension. After confirming the applicability of this method, I went on to compare immature and mature oligodendrocytes. Interestingly, in contrast to immature cells, mature oligodendrocytes that had formed sheets displayed a significantly higher surface tension in the cell areas corresponding to the leading edge compared to the cell body and sheets (Figure 3.17C).

Collectively, this indicates that oligodendrocytes generate high surface tension at the leading edge by locally polymerizing F-actin. This also correlates well with the formerly observed membrane spreading and increase in cell projected areas upon Factin depolymerization since it would lead to a reduction of tension, releasing the excess membrane stores that mature oligodendrocytes build up during differentiation.

\subsubsection{The actin depolymerizing factors ADF and Cofilin1 are responsible for low F- to G- actin ratios}

Based on my previous observations, we propose that F-actin polymerization at the leading edge is the driving force for its forward extension growth. In order for a compact myelin sheath to be formed, an actin depolymerization phase has to take place for the membrane to spread right behind the expanding leading edge. For the cell to achieve this, a high F-actin turnover rate given by the ratio between filamentous (F-) and globular (G-) actin is required. Supporting this idea, a transcriptome database search 
(Cahoy et al., 2008) revealed that several gene products involved in F-actin turnover are enriched in oligodendrocytes.

Among those, I focused on the ADF and Colfilin1 proteins because of their upregulation during myelin biogenesis (Figure 3.18). Since both proteins are redundant, single deletions had no neurological defects. Therefore, an ADF-Cofilin1 double knockout (AC DKO) was generated by crossing an $\mathrm{ADF}$ knockout mice $\left(\mathrm{ADF}^{-/-}\right)$with a mouse line in which Cofilin1 was specifically ablated in oligodendrocytes (Cnp1-Cre ${ }^{+/-}$ Cofilin $1^{\mathrm{fl} / \mathrm{fl}}$ ). Mice were born at the expected Mendelian ratio (Figure 3.19A) but developed motor deficits approximately 10 days after birth. From then onwards, the phenotype increased rapidly in severity resulting in hindlimb paralysis, ataxia and tremor. The animals had to be sacrificed at $\sim$ P17. Genotyping was carried out by isolating genomic DNA followed by PCR of ADF, Cnp-Cre and Cofilin1 (Figure 3.19B).
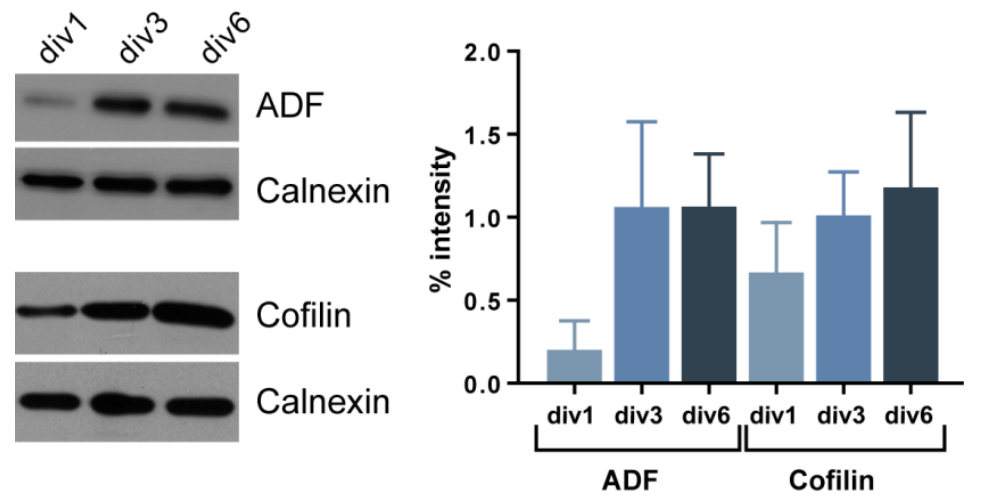

Figure 3.18: ADF and Cofilin1 are upregulated during differentiation. The protein levels of ADF and Cofilin1 were estimated by subjecting cell lysates prepared from oligodendrocytes cultured for one, three and six days in vitro (div) for immunoblotting using ADF, Colfilin and Calnexin specific antibodies. The latter served as a loading control. Quantification shows an increase of band intensity normalized to Calnexin. Graph shows mean $\pm \mathrm{SD}$ ( $\mathrm{n}=3$ independent experiments, one-way ANOVA test; ADF: $* \mathrm{p}<0.05$; Cofilin: n.s.). [Figure kindly provided by Schanila Nawaz]. 
A

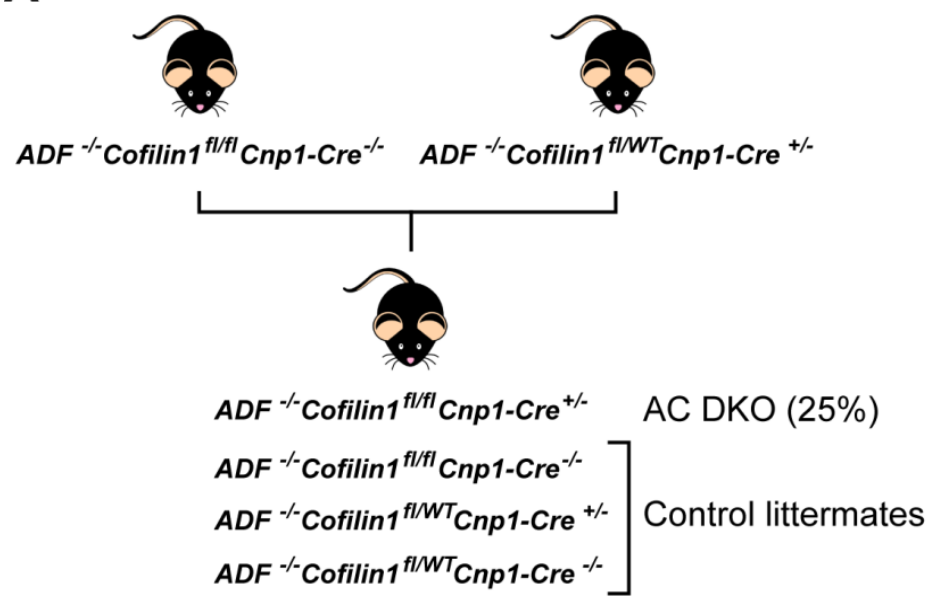

B
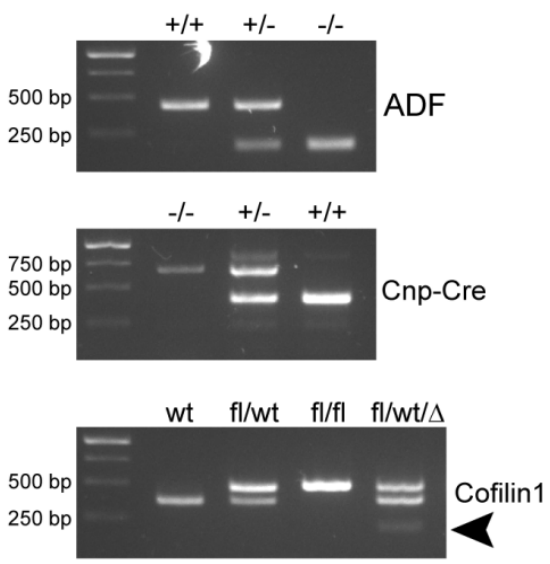

Figure 3.19: Generation and genotyping of ADF-Cofilin1 KO (AC DKO) mice. (A) Generation of AC DKO mice was done by crossing ADF KO with oligodendroglial-targeted Cofilin1 KO. Breeding scheme shows the best pair for getting the highest number of DKO animals per litter. (B) Animals were genotyped using PCR of genomic DNA for 3 genes, ADF, CNP-Cre and Cofilin1. The Cofilin PCR reveals a specific deletion band when the protein Cre is expressed (fl/ wt $/ \Delta$, arrowhead).

I then proceeded to investigate the actin distribution and turnover in the ADFCofilin1 double knockout (AC DKO) mouse line. Using primary oligodendrocytes of control and AC DKO mice subjected to immunocytochemistry, a striking increase of Factin at the leading edge of double knockout mice was observed accompanied by a decrease of cell projected area that can be partially rescued by depolymerizing F-actin (Figure 3.20A). Furthermore, I looked at actin turnover given by the $\mathrm{F} / \mathrm{G}$ actin ratio, and observed that the ratio decreases in control cells during differentiation upon sheet formation (Figure 3.20B), confirming that actin depolymerization is indeed taking place over the course of differentiation. Interestingly, primary oligodendrocytes from double knockout mice already displayed a higher $\mathrm{F} / \mathrm{G}$ actin ratio at the beginning stage of process extension. Upon sheet formation, when sheets are formed, this ratio decreased but still remained elevated as compared to control oligodendrocytes (Figure 3.20B) suggesting that there are alterations in actin distribution and turnover upon genetic deletion of the two actin regulators ADF and Cofilin1. 
A
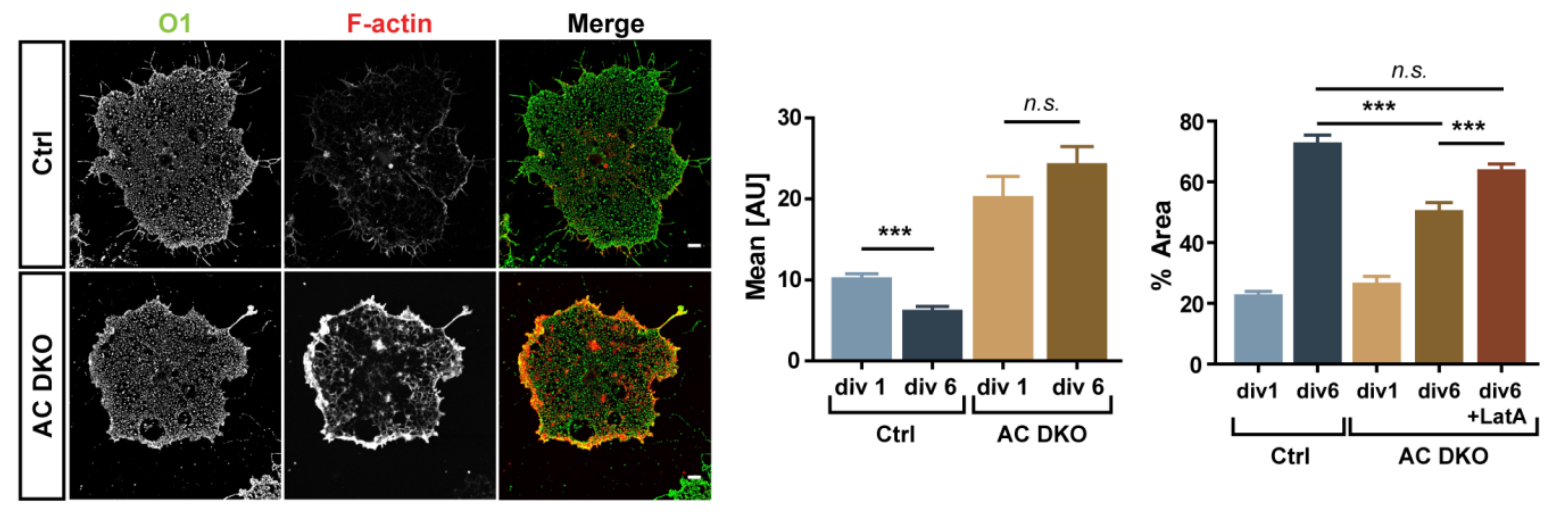

B
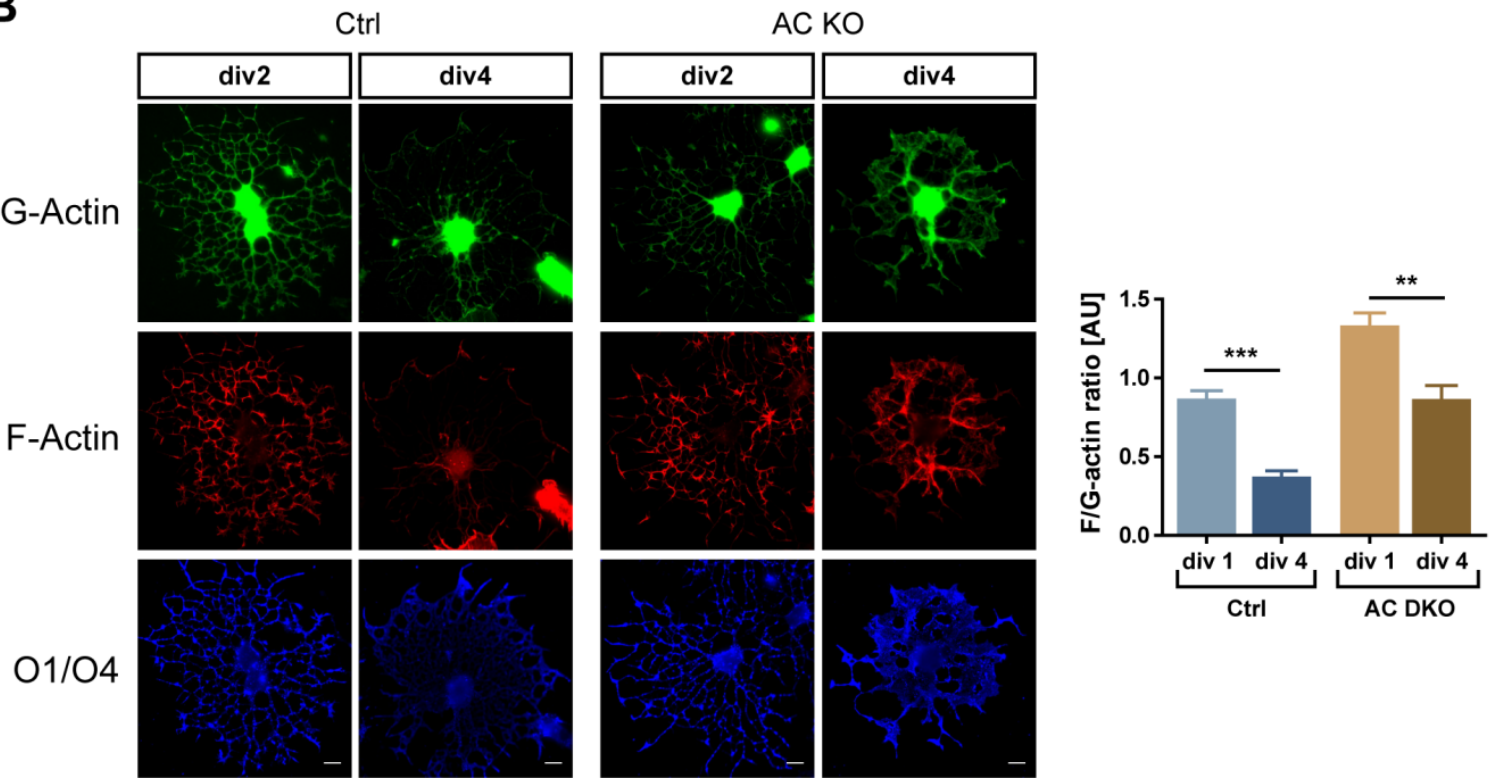

Figure 3.20: F-actin localization at the leading edge and F- to G-actin ratios are significantly higher in ADF-Cofilin1 DKO mice. (A) Primary oligodendrocytes cultured from ADF/Cofilin1 KO (AC DKO) and littermate control mice were stained for F-actin using Phalloidin-rhodamine and with antibodies against galactosylceramide $(\mathrm{O} 1)$ to visualize the cell surface. Quantification shows an increase of F-actin fluorescence intensity in AC DKO cultures, as well as a smaller cell projected area, which can be rescued by depolymerizing actin with LatA. Graphs show mean \pm SEM $(n=26-52$ cells from three experiments, $* * \mathrm{p}<0.01, * * * \mathrm{p}<0.001, \mathrm{t}$-test). Scale bars $=10 \mu \mathrm{m}$. [Figure kindly provided by Schanila Nawaz] (B) Primary oligodendrocytes cultured for 2 and 4 days. DNaseI was used to label G-actin and Phalloidinrhodamine was used to label F-actin. Quantification shows a decrease in F- to G-actin ratio upon differentiation in control animals. F/G actin ratios are significantly higher in cells cultured from AC DKO animals. Graph shows mean $\pm \operatorname{SEM}(\mathrm{n}=17-40$ cells from 3 Ctrl and 3 DKO animals, $* * \mathrm{p}<0.01$, $* * * p<0.001$, t-test). Scale bars $=10 \mu \mathrm{m}$.

Next, due to the fact that ADF-Cofilin1 DKO animals do not survive past P17, I focused on the white matter in the spinal cord since it is among the first areas in the 
CNS to be myelinated in the CNS. At P12, when the most active phase of myelin growth is completed, F-actin was almost undetectable in spinal cord cross-sections of control animals (Figure 3.21). In the AC DKO animals, however, there was a dramatic increase of F-actin levels at the inner tongue, also referred as the leading edge of the myelin sheath (Figure 3.21).
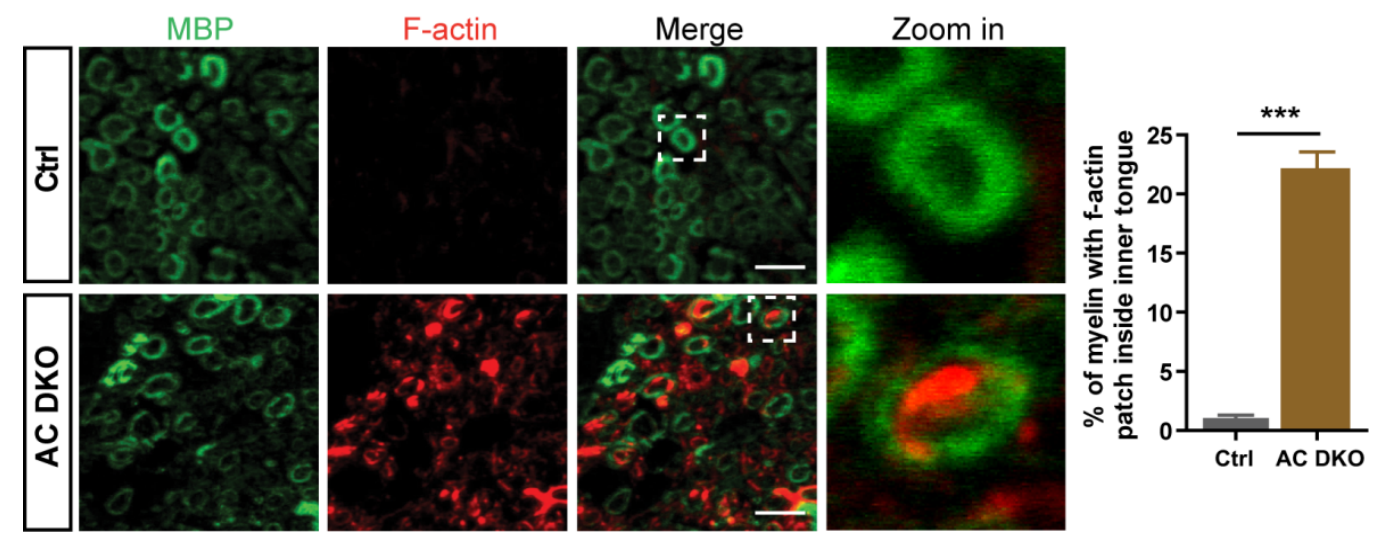

Figure 3.21: Increase of F-actin levels located at the leading edge of ADF Cofilin1 DKO mice. Factin and MBP localization in $400 \mathrm{~nm}$ thick spinal cord sections of P12 control and AC DKO mice. Quantification shows an increase of the percentage of myelinated axons with an actin labeling within the inner tongue. Graph shows mean \pm SEM $(n=874-1266$ myelinated axons from 3 Ctrl and 3 DKO animals, ${ }^{* * *}<0.001$, t-test). Scale bars $=5 \mu \mathrm{m}$. [Staining and imaging by Nicolas Snaidero].

Next, I wondered whether the $\mathrm{F} / \mathrm{G}$ actin ratios were also affected. To test this, I investigated spinal cord sections from P11 mice and observed on one hand a higher F/G actin ratio in the white matter of $\mathrm{AC} \mathrm{DKO}$ compared to control animals and on the other hand no increase in the $\mathrm{F} / \mathrm{G}$ actin ratio of grey matter (Figure 3.22). 

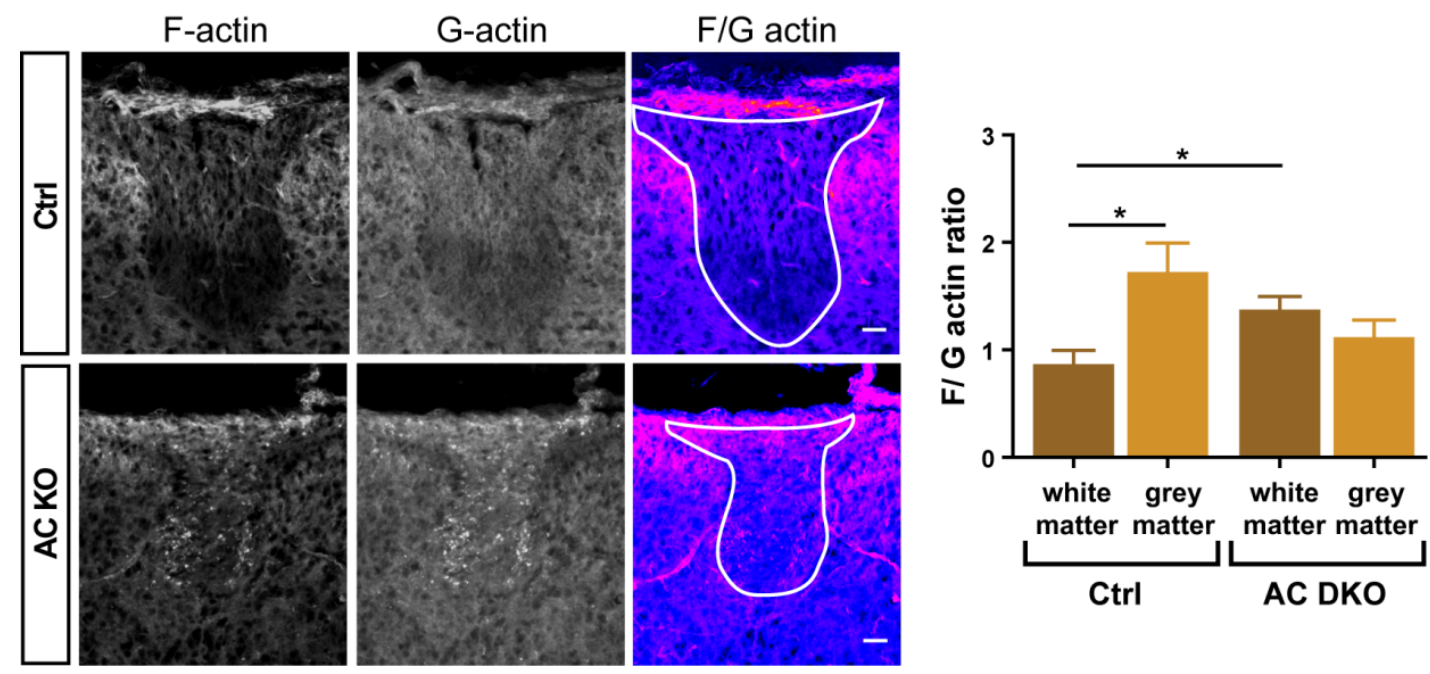

Figure 3.22: F/G actin ratio of ADF-Cofilin1 DKO animals is higher as compared to controls. F- and G- actin staining on $25 \mathrm{~mm}$ spinal cord sections of control and AC DKO mice at P11. F/G actin ratio is shown in pseudocolors to display the intensity ratios more clearly. Quantification shows that the ratio in white matter (area demarked by the continuous white line) of AC DKO mice is higher compared to the controls and is similar to the ratio from grey matter. Graph shows mean \pm SEM $(n>10$ images from 4 animals, ${ }^{*} \mathrm{p}<0.05$, t-test). Scale bars $=10 \mu \mathrm{m}$.

Taken together, these results demonstrate a high F-actin disassembly rate during myelination and identify the actin disassembly factors ADF and Cofilin1 as the crucial actin regulators in vivo.

\subsubsection{ADF and Cofilin1 are required for myelin growth but not for its maintenance}

Assuming that high actin turnover rates are required for myelin wrapping along the axon, one would expect that AC DKO animals would display thinner myelin sheets. In order to test this, electron microscopy (EM) was used to compare spinal cords of animals at P7, P13 and P17 (Figure 3.23). At P7, relatively normal levels of myelination with no difference in myelin thickness or number of myelinated axons were observed between control and AC DKO mice (Figure 3.23A-B). Later, at P13 and P17, several defects in the ultrastructure of myelin could be observed including a lower thickness 
and a decrease in the number of myelinated axons in the AC DKO compared with controls (Figure 3.23A-B). Furthermore, the size of the inner tongue leading edge in the AC DKO mice was increased (Figure 3.23C). These results indicate that myelin sheath growth is stalled in ADF-Cofilin1 DKO animals after P7 with only a minor increase in the number of myelinated axons. Furthermore, these results show that actin depolymerization may be required to control not only myelin sheath growth but interestingly also the size of the inner tongue.

A
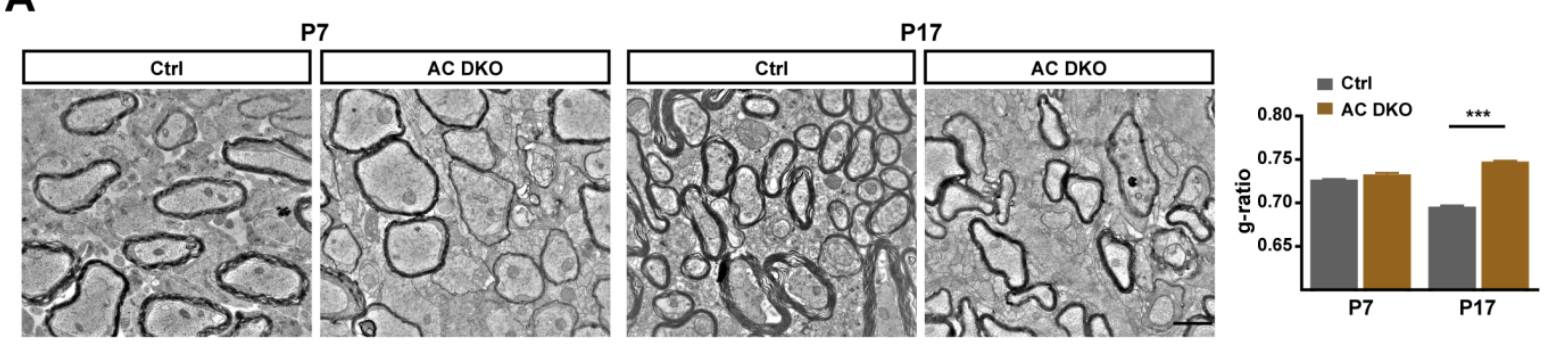

B

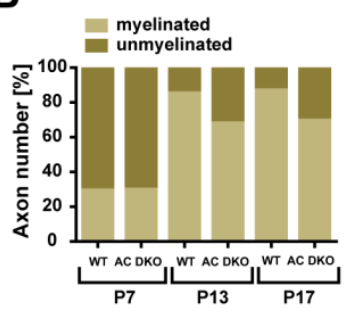

C
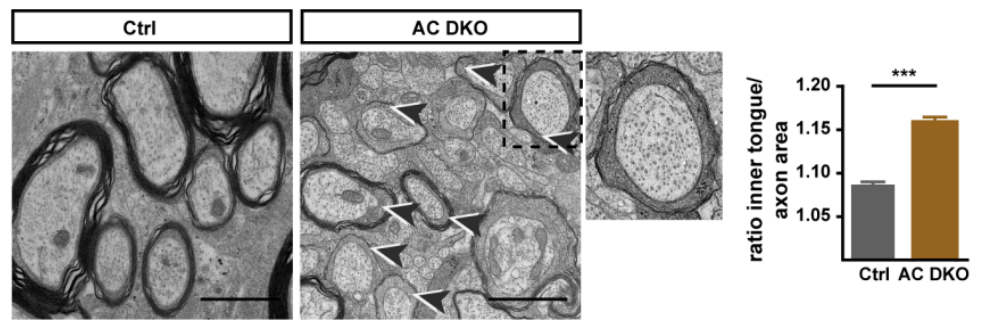

Figure 3.23: ADF/Cofilin1 are required for myelin biogenesis in mice. (A) Electron micrographs of cervical spinal cord from control and AC DKO mice at P7 and P17. Quantification shows the average gratio indicating that myelin thickness increases in control but not in AC DKO animals. Graph shows mean \pm SEM ( $\mathrm{n} \sim 100$ axons from $3-4$ animals of each age and genotype, $* * * \mathrm{p}<0.001$, t-test). Scale bar $=1$ $\mu \mathrm{m}$. [Sample processing and imaging by Nicolas Snaidero and Caroline Velte, quantification by Schanila Nawaz] (B) Percentage of myelinated and unmyelinated axons from P7, P13 and P17 animals shows that the AC DKO animals have less myelinated axons from P13 onwards compared with controls ( $\mathrm{n} \sim 1000$ axons from $>50$ EM images of 3-4 animals per time point). [Sample processing and imaging by Nicolas Snaidero and Caroline Velte, quantification by Schanila Nawaz] (C) AC DKO shows increased inner tongues (arrow heads and inset) compared to control animals. Quantification shows a larger inner tongue diameter in relation to the axonal size in AC DKO animals at P17. A ratio of 1 corresponds to no inner tongue. Scale bar $=1 \mu \mathrm{m}$. [Sample processing and imaging by Nicolas Snaidero and Caroline Velte, quantification by Schanila Nawaz]. 
The defects in myelin sheath growth could either be due to a failure in the wrapping and flattening processes or due to a decrease in the number of precursor and mature oligodendrocytes. To test this, I stained P11 spinal cord sections with antibodies against precursor and mature cells and found that there was no difference between control and AC DKO mice (Figure 3.24). These results demonstrate that the defects in myelination are not due to alterations in the number of myelinating cells but rather a direct effect of the actin alterations in the leading edge.

A
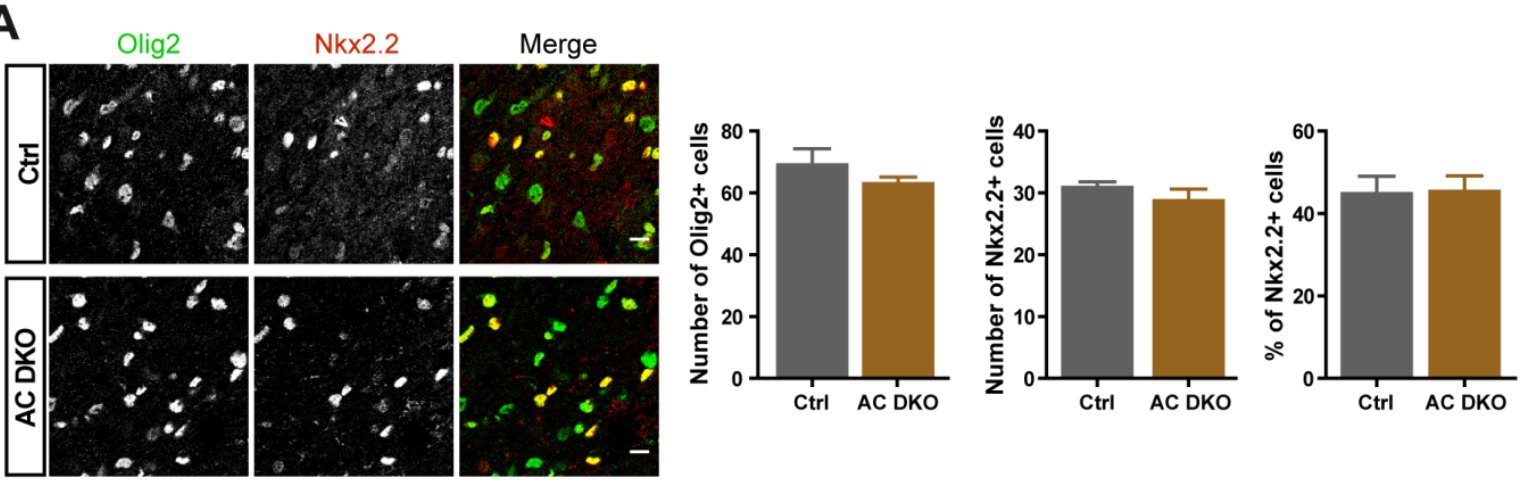

B
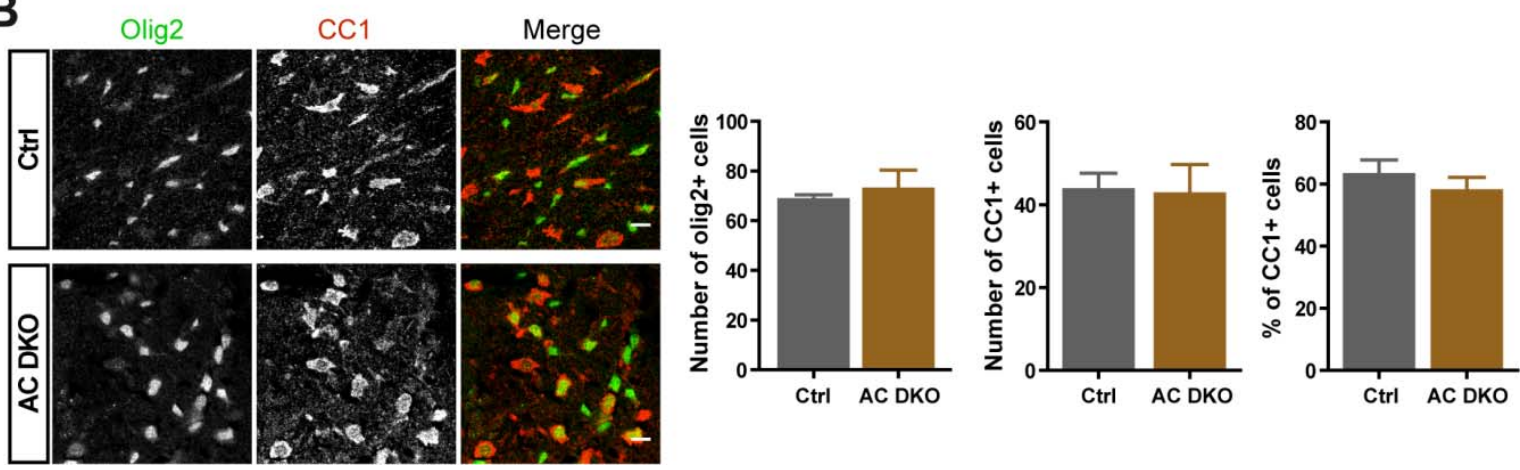

Figure 3.24: The number of oligodendroglial cells between control and ADF/Cofilin1 DKO mice is comparable. $(\mathrm{A}, \mathrm{B}) 10 \mu \mathrm{m}$ spinal cord sections of control and AC DKO mice at P11 were stained for Olig2 (A,B), Nkx2.2 (A) and CC1 (B). Quantification shows that the number of positive cells per area in the white matter remains the same between control and AC DKO mice. Graph shows mean $\pm \operatorname{SEM}(n=2$ sections per animal, 3 animals per genotype. A total area of $33500 \mu \mathrm{m}^{2}$ divided into 5 different regions was measured on each section). Scale bars $=10 \mu \mathrm{m}$.

Finally, we asked whether ADF and Cofilin are also required for the maintenance of the myelin sheath after completion of the developmental process. For this, tamoxifen-inducible conditional double knockout mice were generated by crossing 
$\mathrm{ADF}^{-/}$with Cofilin ${ }^{\mathrm{fl} / \mathrm{fl}} \mathrm{Plp} 1-\mathrm{CreERT} 2$ mice. The animals were induced at P21, when myelination in the spinal cord is complete followed by analysis 9 weeks later. No difference in the number of myelinated axons or in myelin thickness was observed (Figure 3.25).
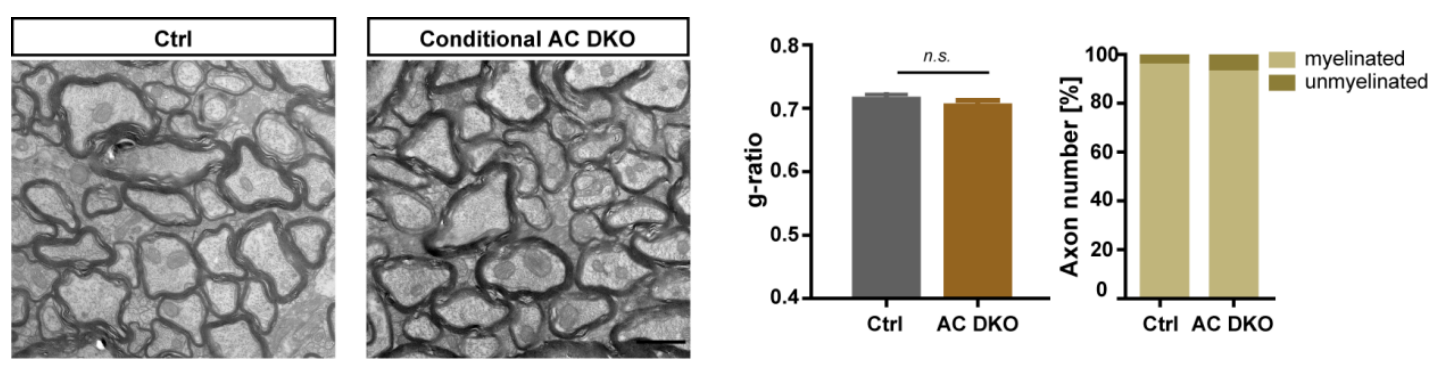

Figure 3.25: Inducible ADF-Cofilin DKO mice show no difference in myelin compared with control animals. Electron micrographs of tamoxifen-inducible AC DKO. Animals were injected with tamoxifen at P21 and analyzed 9 weeks later. Quantification shows that the g-ratios and percentage of myelinated and non-myelnated axons is the same for both. Graph shows mean \pm SEM ( $\mathrm{n} \sim 400$ axons from 3 animals of each genotype). Scale bar $=2 \mu \mathrm{m}$. [Sample processing and imaging by Nicolas Snaidero and Caroline Velte, quantification by Schanila Nawaz].

Collectively, these results suggest a major role of actin turnover in myelin sheath formation by controlling the motility of the leading edge and the spreading of membrane identifying the actin depolymerization factors ADF and Cofilin as main regulators. 
$80 \mid$ Results 


\section{\begin{tabular}{l|l} 
Discussion & 4
\end{tabular}}

\subsection{Validation of a vertical optical trap for mechanical measurements}

In this first part of the work I have shown that a custom-built vertical optical trap setup can be used to perform mechanical measurements on cells. Furthermore, the response of cells to mechanical deformations was found to be visco-elastic, and depended on both the length and directionality of deformation as well as on the presence of the actin cytoskeleton.

The response of cells to mechanical stress in an experimental setup depends on many parameters, such as the time and length scales of the measurements. These parameters cannot always be freely chosen but depend strongly on the technique that is used to deform the cell. From all techniques, AFM is the most widely used for measuring cell stiffness by recording the response of single cells upon indentation. The amount of force that an AFM setup can apply to the cell depends on the spring constant of the cantilever and the force set point determined by the user. The contact mechanics during the indentation depend on the shape of the AFM tip, which can be varied. Sharp tips, for example, focus the applied force on a very small area of the cell surface thereby increasing the local stress and the probability to induce rupture if the force is high enough. Alternatively, very blunt tips such as micrometer sized beads glued to end of 
the cantilever, can be used to distribute the force over a larger surface. Thus, the local stress on the membrane is reduced which lowers the risk of disrupting it. If the wrong parameters are used AFM can easily exert forces large enough to damage the cells which consequently will result in measurement artifacts. These could lead then to misinterpretation of the state of the biological sample, since e.g. apoptotic cells show lower stiffness than healthy cells (Hu et al., 2009; Pelling et al., 2009). Besides using blunt probes to deform the cell, an alternative solution to this problem would be to simply decrease the applied force. However, the minimum force that can be applied is limited by the intrinsic noise of conventional AFM cantilevers given by its thermal fluctuations and it reaches values around $10 \mathrm{pN}$ (Dey and Szoszkiewicz, 2012). This does not only limit the minimum force that can be applied to tens of $\mathrm{pN}$ but it also creates a problem for the analysis of the indentation curves. The low force AFM curves as presented in Fig. 3.2 $\mathrm{C}$ show that the noise makes it very difficult to accurately determine the point when the AFM contacts the cell. Defining the right contact point is crucial to define the absolute amount of deformation, which, as we have found, has a large influence on the measured Young's modulus of the cell (Eq. 9). Efforts to limit the forces and deformations are particularly relevant in this project because the main goal of this study is to measure mechanical properties of oligodendrocytes that form very flat structures which obviously cannot sustain very large indentation.

To lower the above mentioned force noise and the resulting complications we have designed an optical trap that can be controlled in the vertical (z) direction (Bodensiek et al., 2013). This is a novel approach since most optical traps move in the direction parallel to the surface $(\mathrm{x}, \mathrm{y})$. Moving in the $\mathrm{z}$ direction has the advantage of having the same boundary conditions as an AFM. The cell is supported by a planar surface and indented from above with a probe which simplifies the modeling of the cell 
deformation. In fact, the same Hertz model used for calculation of the Young's modulus when using AFM can be applied. The main advantage of optical traps is the very low force noise of less $1 \mathrm{pN}$ which is due to the much lower spring constant of the optical trap of typically $0.1 \mathrm{pN} / \mathrm{nm}$ (whereas even a very soft AFM cantilever has a spring constant of at least $10 \mathrm{pN} / \mathrm{nm}$ ) (Bodensiek et al., 2013). This advantage combined with the vertical operation of our vertical optical trap enabled me to measure cells mechanics at forces lower than $10 \mathrm{pN}$, corresponding to an indentation of $\sim 250 \mathrm{~nm}$. This is substantially lower than the indentation applied by AFM setups which typically deforms for about a micrometer. Also, the force versus deformation curves acquired with the vertical trap such as presented in fig. 3.2 D show clearly that the contact point between the bead and the cell can be much more accurately determined. One disadvantage of optical traps is the need for a high power laser source, which can lead to an increase in temperature of a few degreed near the focus (Peterman et al., 2003). In the case of our vertical trap, this could be a potential issue since the light beam penetrates the sample and passes though the cell thereby potentially damaging it. However, the short time scale of the measurements, typically being less than 2 minutes per cell, and the fact the laser is focused outside and away of the cell, minimize this potential risk. Also, control measurements where we positioned the laser focus just outside of the cell periphery for 10 minutes (about 10 times the duration of the indentation experiment) did not result in any sign of stress from the cell (Nawaz et al., 2012).

Overall, our vertical optical trap has proven to be a competent method to perform accurate force spectroscopy measurements on cells using low forces and permitting smaller indentations as compared to AFM and thus making it a suitable tool for the measurement of very flat cells with minimal cell damage and influence of the underlying substrate. 


\subsection{The response of cells to small deformations is elastic but anisotropic}

The measurements of cell elasticity using our vertical trap at small indentations of $\sim 200 \mathrm{~nm}$ revealed an almost ideal elastic response. This is evidenced by: 1) the absence of hysteresis (and thus a low amount of energy lost) between the indentation and retraction curves, and 2) the observation that the obtained Young's modulus of $100 \mathrm{~Pa}$ is independent on the rate of deformation. The vertical optical trap used for this study initially provided only a limited range of velocities. After the optimization of the fast force feedback loop we were able to produce a response in milliseconds and thereby moving the bead at speeds up to $50 \mu \mathrm{m} / \mathrm{s}$ during indentation measurements (Bodensiek et al., 2013). Using this improved setup, we confirmed that the elastic modulus of fibroblasts also remained constant over a wider range of speeds (Bodensiek et al., 2013), further supporting our finding that the cell response is elastic at small deformations. The same speed-independent elastic modulus was obtained from AFM measurements analyzed at forces up to $30 \mathrm{pN}$ which confirms an elastic response at small deformations. In this case the obtained Young's modulus was $85 \mathrm{~Pa}$ which shows that our optical trap setup produces results that are comparable with those of an AFM setup and that the observation of an elastic regime is independent on the measurement technique.

Although both AFM and optical trapping delivered consistent results for the Young's modulus, the values reported in literature are 10 to 100 times higher, as reviewed in (Kuznetsova et al., 2007; Vinckier and Semenza, 1998). Since almost all AFM experiments are performed at much higher forces and indentations than what we used, this discrepancy may largely result from the observed increase in apparent stiffness at 
larger deformations due to the presence of a viscous component, which will be further discussed in section 4.4 .

Because the cells respond elastic at small indentations one would expect that changing the direction of deformation (stretching vs. indenting) would not affect the measured Young's modulus. Surprisingly, when using the vertical trap for stretching the cells, the calculated Young's modulus was more than twice as high (239 Pa) than the estimated modulus from indentations (100 Pa). This difference could only be partially attributed to the difficulty of defining specific contact conditions of the experiment, more specifically the estimation of the exact contact area between the membrane and the bead which would have an impact on the calculated Young's modulus. The assumption was that the maximum contact area is reached during the preceding indentation and remains constant throughout the pulling. It is however possible that low adhesive forces potentially result in a decreased contact area and thus a too high calculated value for the Young's modulus, or an increase of the contact area due to very high adhesion which would lead to a too low Young's modulus. Nonetheless, even if an error of $50 \%$ in the contact area estimation is assumed, the calculated values range from 160 to $480 \mathrm{~Pa}$ which is still higher than the values obtained from the cell indentation experiments (Nawaz et al., 2012). The most favorable explanation for this behavior is the anisotropy of the membrane-cortex interaction (Hu et al., 2003). Similar anisotropic results have been previously found, although in the opposite direction (Acerbi et al., 2012), where indenting lung fibroblasts resulted in a 6 times higher Young's modulus as compared to stretching them at high deformations. 


\subsection{The elastic response of cells is determined by the actin cortex.}

The amount of deformation of a cell is determined by its intracellular structures. At very low forces, the plasma membrane and the underlying acting cortex are the main contributing factors for the calculated Young's modulus while at deeper indentations the influence of the cytoplasm and cellular organelles start to play an increasing role. The effect of the cytoskeleton on the elastic modulus of cells has been described before by measuring fibroblasts with AFM at forces between $160-400 \mathrm{pN}$ upon treatments that individually disrupt particular filaments of the cytoskeleton, such as actin and microtubules (Rotsch and Radmacher, 2000). Actin filament stabilization or microtubule disruption did not alter the measured Young's modulus. However, the elasticity of the cells decreased more than a two-fold upon actin depolymerization and disruption of stress fibers, demonstrating its dependency on the actin filaments (Rotsch and Radmacher, 2000). My results when measuring the effects of actin depolymerization were obtained with the vertical optical trap at much lower forces and smaller indentations. Upon actin depolymerization, the Young's modulus decreased more than a three-fold from 100 to $29 \mathrm{~Pa}$. This confirms the cell cortex as the main contributor to cell elasticity and shows that its influence at small deformations is even stronger than at larger deformations.

\subsection{The response of cells to large deformations has a major viscous component}

When AFM indentation experiments are performed at higher forces and indentation, this resulted in an increase in hysteresis (and thus the amount of energy dissipated by 
the cell). Additionally, the apparent Young's modulus increased (from 140 to $330 \mathrm{~Pa}$ ) at higher rates of deformation which is a characteristic behavior of a viscous response. To identify the type of viscosity, the apparent Young's modulus against the indentation rate values was plotted on a double logarithmic scale and fitting with a power function (Alcaraz et al., 2003; Balland et al., 2005; Hoffman et al., 2006). If a substance is solid with an ideal elastic behavior, the exponent will be 0 . If the measured substance is a liquid and behaves like an ideal viscous material, the slope will be 1 . Our measurements showed an exponent between 0 and 1 which clearly show that cells exhibit both elastic and viscous behavior. Interestingly the measured slope depends on how the measurement is performed. At very small indentations with the optical trap the response was elastic which corresponds to an exponent of 0 . At larger indentations of around 0.3 $\mu \mathrm{m}$ the exponent was 0.17 while this increased to 0.31 at indentations of around $1 \mu \mathrm{m}$ (Figure 3.5). These finding are consistent with literature which reports that frequencydependent stiffness values follow a weak power law that depends on the type of deformation. Cells probed either in the proximity of the cell cortex or deeper into the cytosolic space yield an exponent value that increased from 0.13 to 0.29 (Hoffman and Crocker, 2009; Hofmann et al., 1997). These literature values were obtained with many different techniques and dependent on the type of technique the measurements were either sensitive to the cell periphery and yielded low values for the exponent, or the measurements were sensitive to the interior of the cell and yielded a higher value for the exponent. The uniqueness of our results is that we could extract the different exponents by just analyzing different indentation depths of the same AFM indentation curves. So with a single technique we can access different parts of the cell which unambiguously confirms the existence of different visco-elastic environments in a single cell. The reason for the variable exponent follows from the inhomogeneous composition of the 
cell. The cell cortex responds mainly elastically whereas the deeper areas such as cytoplasm and nucleus respond more viscous.

\subsection{Actin distribution during myelination}

In the second part of my work I used diverse approaches to study primary oligodendrocytes and with the support of animal models, we have gathered new insights on the importance of actin dynamics for proper myelination.

F-actin has been shown to be located in the inner and outer mesaxon, SchmidtLanterman incisure, periaxonal membrane and paranodal loop in Schwann cells, responsible for myelin formation in the PNS (Trapp et al., 1989). In these cells it is also required for maintaining shape and gene expression related to myelination (FernandezValle et al., 1997). In oligodendrocytes, the distribution of F-actin has a very particular distinct pattern which varies depending on the cell differentiation stage. However, it is consistently present at the growing edges of cells. At early stages of differentiation in vitro, actin is initially enriched in a growth cone-like structure at the end of the cell processes. Later on, it is almost entirely depleted from the sheets, but remains localized at the outer rim of the growing sheet. In vivo, it is located in the uncompacted inner tongue during active growth (Figure 3.8); (Dyer and Benjamins, 1989; Snaidero et al., 2014). Strikingly, upon termination of myelination, F-actin disappears from the myelin sheath both in vitro and in vivo (Figure 3.8); (Snaidero et al., 2014).

Since in vivo the inner tongue is inaccessible because it is situated within the multilayered membrane, these changes in actin localization and its effects on oligodendrocyte morphology can be investigated better by using primary oligodendrocyte cultures. In this simplified system, it then becomes necessary to define 
the correlates of the in vivo structures, in particular the leading edge. Figure 4.1 illustrates the growth zones, also referred to as the leading edge, at each stage of development. They are characterized by the presence of F-actin which at the branching stage is limited to the growth cone. Afterwards, the sheets are being formed in between the processes, expanding towards each other thus filling up the gaps. At the end, the motile part of the growth zone is restricted to the outer rim of the cell showing back and forth movements.

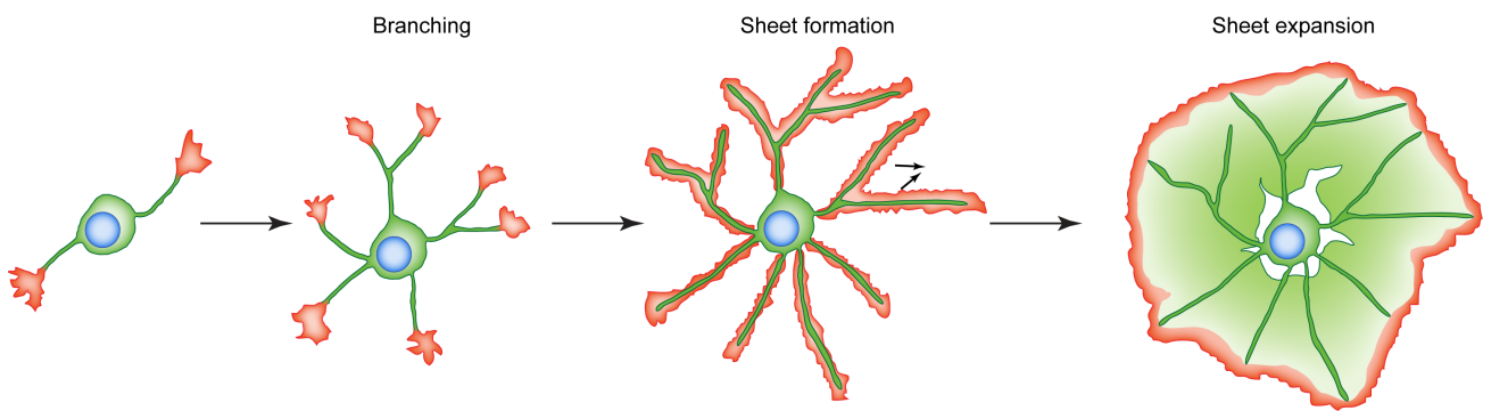

Figure 4.1: Leading edge in oligodendrocytes at different stages of differentiation. Illustration showing oligodendrocyte processes in green and the growth zones in red. The arrows indicate the direction of membrane expansion within the growing sheet.

\subsection{Shift between two migratory mechanisms allows wrapping of the leading edge}

The results obtained from primary oligodendrocytes in this study demonstrate that oligodendrocytes have two different mechanisms of leading edge extension. First, an adhesion-based mechanism comparable to the neuronal growth cones. This involves adhesive molecules at its base connecting the cytoskeleton with proteins of the ECM thus generating force transmission, a lamellipodium at the periphery rich in actin pushing forward membrane protrusions by actin polymerization and dynamic filopodia extending beyond the membrane. Once myelin sheet formation begins, the leading edge is no longer adhesive evidenced by the lack focal adhesion components but is instead 
displaying highly dynamic characteristics, as demonstrated by using IRM. Contrary to the actin-rich leading edge of processes, which moves in a targeted direction, the leading edge of sheet forming oligodendrocytes randomly extends protrusions in every direction which does not result in a net forward movement.

Migration independent of integrins and, therefore, of adhesive forces has been described for leukocytes, in which cortical actin and myosin-IIA-dependent contractility are responsible for generating protrusions that only under confinement conditions are able to generate friction, overcome the resistance and subsequently squeeze in between tight spaces (Krummel et al., 2014; Lammermann et al., 2008; Renkawitz and Sixt, 2010). Moreover, they are capable of adapting by switching from a mesenchymal, integrin-based into an amoeboid integrin-independent migration based on the adhesive properties of its environment (Krummel et al., 2014; Renkawitz et al., 2009).

Recently, this switch between mesenchymal- and deformation-based migration in a 3D environment has also been described for other types of cells such as the migratory progenitor cells in zebrafish gastrulation, where the trigger for switching is based on an increase in contractility (Ruprecht et al., 2015) or confinement conditions with a mechanism mediated either by generation of local protrusions or by myosinII-dependent contractility (Liu et al., 2015). Due to the increased appearance of focal adhesions evidenced by vinculin staining at early differentiation stages, I have provided evidence that oligodendrocytes are capable of using a mesenchymal mechanism for movement and, at later stages of differentiation, develop a deformation-based mechanism of process locomotion by lowering their membrane tension only depending of F-actin depolymerization and a decrease in adhesion complexes. The low tension together with its independence of myosin activity as evidenced by no effects on membrane tension in the presence of the myosin inhibitor blebbistatin, rules out any mechanism based on 
actomyosin contractility thereby suggesting that instead, their motility is based on extending protrusions only by local actin polymerization. Furthermore, our hypothesis is supported by the observed changes in $\mathrm{F} / \mathrm{G}$ actin ratios in mouse primary oligodendrocyte cultures and spinal cord tissue favoring the polymerization of actin filaments only at the process formation stage, whereas the equilibrium of $\mathrm{F} / \mathrm{G}$ actin is shifted towards the free monomeric form at later stages, shown by the decrease of Factin staining in the myelinated fibers of the white matter.

\subsection{Membrane spreading as a wetting transition}

Wetting is a term used to describe the spreading of a small liquid drop onto a flat solid surface. It depends mainly on the intrinsic properties of each element which determine the adhesive and repulsive forces between the two surfaces placed in contact. The free energy per unit of area between the solid-vapor, solid-liquid and liquid-vapor interfaces determines the spreading coefficient and the contact angle at which the liquidvapor interface intersects the solid-vapor interface (de Gennes, 1985). A higher spreading coefficient indicates the tendency of a liquid to spread onto a surface with a smaller contact angle when the spreading is larger. Once the two surfaces reach an equilibrium, it can be either described as a partial wetting, where the liquid drop and the surface are in an steady state and the contact angle is constant, or a complete wetting where it has spread until the contact angle is 0 (de Gennes, 1985). Subsequent studies implemented this knowledge into the concept of cell adhesion, in which the wetting transition is determined by a balance between adhesive and repellent forces generated by specific receptors, nonspecific interactions, the glycocalix and membrane elasticity (Sackmann and Bruinsma, 2002). Consequently, spreading will occur if the free energy 
of adhesion towards the surface overcomes the work against it. Furthermore, it has been described that the dynamics of a group of cellular aggregates spreading onto a surface initially shows a collective deformation comparable to a drop with visco-elastic characteristics and afterwards, a thin monolayer of cells spreads on the surface and can migrate away from the aggregate (Douezan et al., 2011).

In oligodendrocytes, as shown in this study, membrane spreading occurs at later stages of differentiation, when adhesive contacts remain no longer established with the surface. Since the adhesive forces must be very low at this stage, there has to be another mechanism by which oligodendrocytes are able to spread their membrane. One possibility is that adhesion would be triggered upon axonal contact by so far unidentified molecules, however the molecules involved are not yet known and since the leading edge displays very dynamic characteristics when wrapping around the axon forming strong adhesive contacts would not be favorable. An explanation of how spreading could happen under low adhesive conditions can be found partially in studies describing the mechanism for tissue segregation during embryogenesis. It was proposed long ago, that the determining property driving cell sorting in tissue was the difference in surface tension between the types of cells (Harris, 1976). According to this, if the surface tension of one cell type is higher than the other one, it will pull the cell type with lower tension around it (Beysens et al., 2000; Foty and Steinberg, 2004; Maitre et al., 2012). Moreover, it has been demonstrated in progenitor cells from zebrafish embryos that the cell-cell interfacial tension is determined by the cortical tension instead of the tension generated by adhesive contacts (Maitre et al., 2012). Oligodendrocytes control their surface tension by F-actin depolymerization (Figure 3.17), which is being reduced it during sheet formation, thereby raising the possibility that it can surround a surface with a higher tension such as the axon. 
Besides a low surface tension, large amounts of membrane have to be available for the extensive spreading to take place. Oligodendrocytes build up a large stock of membrane reservoirs prior to sheet formation that are kept together as infoldings by Factin, as shown in Figure 3.16B similar mechanism of excess membrane storage has been previously described for Drosophila embryos storing membrane in reservoirs located in microvilli (Figard et al., 2013). The first 13 divisions of the Drosophila embryo take place without cytokinesis followed by plasma membrane ingressing around every nucleus to form the individual cells in one cell cycle, increasing the surface area $\sim 25$ fold (Figard et al., 2013). It was shown that microvilli can store at least half of the membrane required for the cellularization thus being the source for the excess membrane and membrane further release (Figard et al., 2013). This shows that infoldings can store large amounts of membrane and supports our hypothesis that membrane reservoirs can be stored in oligodendrocyte processes being released upon before sheet formation.

Taken together, our data suggests that oligodendrocytes spread their membrane by lowering membrane tension through F-actin depolymerization, thus releasing membrane reservoirs in a mechanism following the principles of a wetting transition.

\subsection{The factors ADF/Cofilin1 are necessary for actin depolymerization and myelin growth}

Given the importance of actin turnover in oligodendrocyte differentiation and myelin formation, it was important to identify the factors involved in this regulation. In this study I have described that impaired F-actin depolymerization affects myelin formation in mice with the factors $\mathrm{ADF}$ and Colifilin1 being critical for its regulation. 
Furthermore, the oligodendrocyte developmentally regulates ADF and Cofilin1. This makes sense since actin depolymerization would remove the constrains of the actin cytoskeleton on the membrane resulting in its release and thereby its flattening. The downregulation of vinculin-positive focal adhesions is also linked to this stage, since the forward movement of the oligodendrocyte membrane in between axon and compacting myelin membrane no longer requires traction force generation in attachment with the ECM but instead is achieved by dynamic regulation of the cytoskeleton. In light of this it also becomes clear why ADF and Cofilin1 play a role in myelin formation but not on myelin maintenance because once compacted, myelin is linked by MBP with little or no space and there is no functional relevance for the actin cytoskeleton.

However, the AC DKO mouse model still showed axons with compacted myelin. This could be due to compensation by others existing actin depolymerization factors, e.g. proteins of the gelsolin family (Vouyiouklis and Brophy, 1997). However, due to the lack of two main regulators of actin depolymerization once would expect a decrease on the effectiveness of processes related to it which in the mice could be observed by the increase in inner tongue area and increased $\mathrm{F} / \mathrm{G}$ actin ratios resulting in disturbances of the myelination process. Additionally, it is possible that some cells escape Cre recombination and express Cofilin1, therefore forming normal myelin.

\subsection{Proposed model for the role of actin in myelin formation}

Collectively, we propose that actin turnover plays a major role in myelination, where oligodendrocytes, in an initial stage of process formation, use a mesenchymal mechanism for locomotion. High tension and adhesion to the ECM will generate traction forces that will be transduced through the actin cytoskeleton promoting 
lamellipodium extension and process growth similar to the mechanism described for neuronal growth. Subsequently, they will use forces generated by F-actin polymerization to inflate the leading edge at the inner tongue, pushing it forward throughout the space between the myelin layers and the axonal membrane. F-actin depolymerization at the base of the leading edge regulated by the factors ADF/Cofilin1 favor membrane spreading and adhesion onto the axon in a model resembling wetting transition of liquid droplets (Figure 4.2).

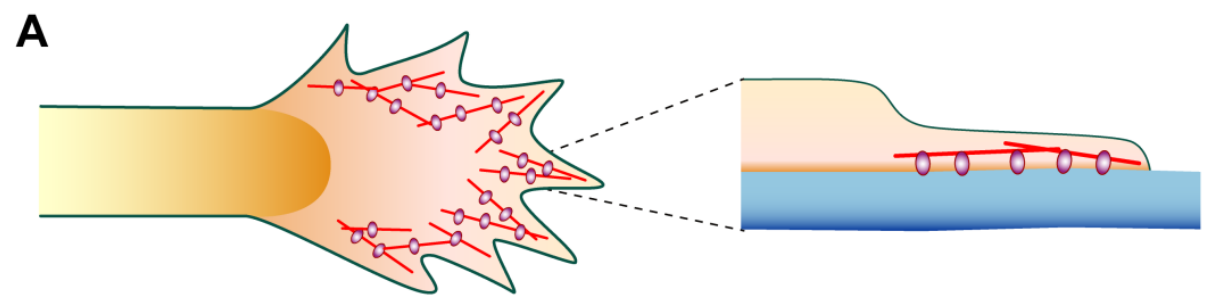

\section{B}

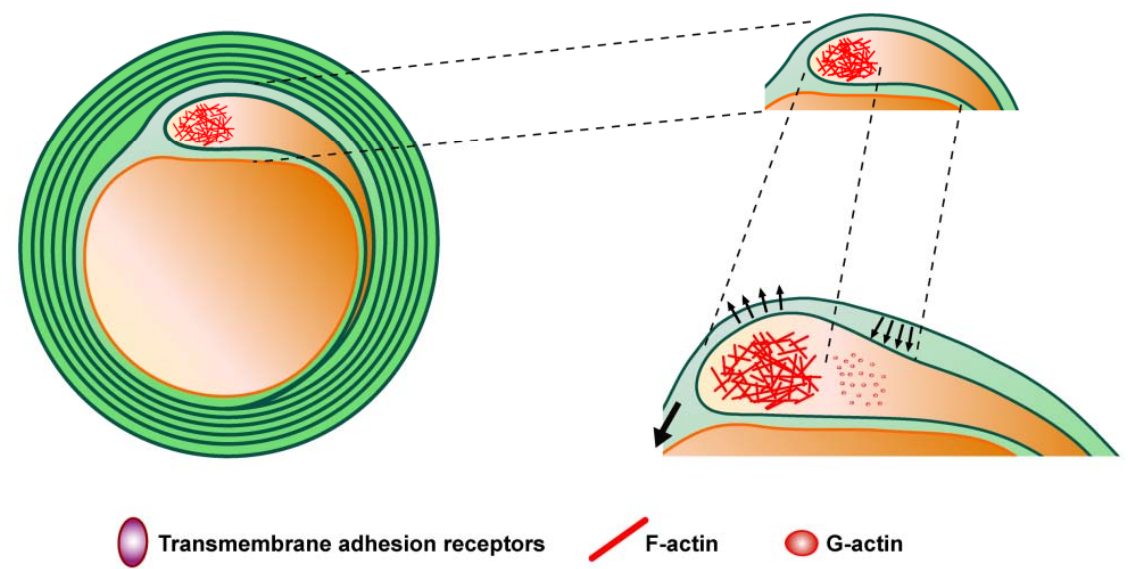

Figure 4.2: Role of F-actin in myelination. (A) Scheme shows the growth cone of an oligodendrocyte precursor, where actin filaments are anchored to the surface by transmembrane adhesion receptors to generate forces large enough to push the leading edge forward. (B) Model showing a process of a myelinating oligodendrocyte using F-actin polymerization forces to inflate the leading edge (arrows pointing upwards) and push it towards the forming myelin sheath and the axonal membrane. Subsequent F-actin disassembly deflates the back of the leading edge (arrows pointing downwards), facilitating spreading and flattening of the membrane to create the newer myelin layers. 
96 | Discussion

In conclusion, this project has delivered new evidence to further elucidate the mechanism of myelin formation in the CNS. To the existent knowledge of the inner tongue as the leading edge and structure responsible for myelin membrane growth around the axon, I have added a novel model describing how the regulation of polymerization and depolymerization of actin filaments controls the motility of the leading edge and membrane flattening. 


\section{References 5}

Acerbi, I., Luque, T., Gimenez, A., Puig, M., Reguart, N., Farre, R., Navajas, D., and Alcaraz, J. (2012). Integrin-specific mechanoresponses to compression and extension probed by cylindrical flat-ended AFM tips in lung cells. PloS one 7, e32261.

Aggarwal, S., Snaidero, N., Pahler, G., Frey, S., Sanchez, P., Zweckstetter, M., Janshoff, A., Schneider, A., Weil, M.T., Schaap, I.A., et al. (2013). Myelin membrane assembly is driven by a phase transition of myelin basic proteins into a cohesive protein meshwork. PLoS biology 11, e1001577.

Aggarwal, S., Yurlova, L., and Simons, M. (2011a). Central nervous system myelin: structure, synthesis and assembly. Trends in cell biology 21, 585-593.

Aggarwal, S., Yurlova, L., Snaidero, N., Reetz, C., Frey, S., Zimmermann, J., Pahler, G., Janshoff, A., Friedrichs, J., Muller, D.J., et al. (2011b). A size barrier limits protein diffusion at the cell surface to generate lipid-rich myelin-membrane sheets. Developmental cell 21, 445-456.

Alberts, B., Johnson, A., Lewis, J., Raff, M., Roberts, K., and Walter, P. (2007). Molecular Biology of the Cell, 6th edition edn (New York, NY: Garland Science, Taylor \& Francis group).

Alcaraz, J., Buscemi, L., Grabulosa, M., Trepat, X., Fabry, B., Farre, R., and Navajas, D. (2003). Microrheology of human lung epithelial cells measured by atomic force microscopy. Biophysical journal 84, 2071-2079.

Allison, D.P., Mortensen, N.P., Sullivan, C.J., and Doktycz, M.J. (2010). Atomic force microscopy of biological samples. Wiley interdisciplinary reviews Nanomedicine and nanobiotechnology 2, 618-634.

Ashkin, A. (1997). Chapter 1 Forces of a Single-Beam Gradient Laser Trap on a Dielectric Sphere in the Ray Optics Regime1. In Methods in Cell Biology, P.S. Michael, ed. (Academic Press), pp. 1-27.

Atanackovic, T.M., and Guran, A. (2000). Theory of Elasticity for Scientists and Engineers (Birkhäuser). 
Balland, M., Richert, A., and Gallet, F. (2005). The dissipative contribution of myosin II in the cytoskeleton dynamics of myoblasts. European biophysics journal : EBJ 34, 255-261.

Barr, V.A., and Bunnell, S.C. (2009). Interference reflection microscopy. Current protocols in cell biology / editorial board, Juan S Bonifacino [et al] Chapter 4, Unit 4 23.

Barres, B.A., and Raff, M.C. (1993). Proliferation of oligodendrocyte precursor cells depends on electrical activity in axons. Nature 361, 258-260.

Baumann, N., and Pham-Dinh, D. (2001). Biology of oligodendrocyte and myelin in the mammalian central nervous system. Physiological reviews 81, 871-927.

Bellenchi, G.C., Gurniak, C.B., Perlas, E., Middei, S., Ammassari-Teule, M., and Witke, W. (2007). N-cofilin is associated with neuronal migration disorders and cell cycle control in the cerebral cortex. Genes \& development 21, 2347-2357.

Beysens, D.A., Forgacs, G., and Glazier, J.A. (2000). Cell sorting is analogous to phase ordering in fluids. Proceedings of the National Academy of Sciences of the United States of America 97, 9467-9471.

Binnig, G., Quate, C.F., and Gerber, C. (1986). Atomic Force Microscope. Physical Review Letters 56, 930-933.

Bodensiek, K., Li, W., Sanchez, P., Nawaz, S., and Schaap, I.A. (2013). A high-speed vertical optical trap for the mechanical testing of living cells at piconewton forces. The Review of scientific instruments 84, 113707.

Bozzali, M., and Wrabetz, L. (2004). Axonal signals and oligodendrocyte differentiation. Neurochemical research 29, 979-988.

Bradl, M., and Lassmann, H. (2010). Oligodendrocytes: biology and pathology. Acta neuropathologica 119, 37-53.

Bunge, R.P., Bunge, M.B., and Bates, M. (1989). Movements of the Schwann cell nucleus implicate progression of the inner (axon-related) Schwann cell process during myelination. The Journal of cell biology 109, 273-284.

Cahoy, J.D., Emery, B., Kaushal, A., Foo, L.C., Zamanian, J.L., Christopherson, K.S., Xing, Y., Lubischer, J.L., Krieg, P.A., Krupenko, S.A., et al. (2008). A transcriptome database for astrocytes, neurons, and oligodendrocytes: a new resource for understanding brain development and function. The Journal of neuroscience : the official journal of the Society for Neuroscience 28, 264-278.

Chhabra, E.S., and Higgs, H.N. (2007). The many faces of actin: matching assembly factors with cellular structures. Nature cell biology 9, 1110-1121.

Christopher R. Jacobs, H.H., Ronald Y. Kwon (2012). Introduction to Cell Mechanics and Mechanobiology. Chapter 1. 
Clark, A.G., and Paluch, E. (2011). Mechanics and regulation of cell shape during the cell cycle. Results and problems in cell differentiation 53, 31-73.

Colognato, H., Baron, W., Avellana-Adalid, V., Relvas, J.B., Baron-Van Evercooren, A., Georges-Labouesse, E., and ffrench-Constant, C. (2002). CNS integrins switch growth factor signalling to promote target-dependent survival. Nature cell biology 4 , 833-841.

Dai, J., and Sheetz, M.P. (1997). Chapter 9 Cell Membrane Mechanics. In Methods in Cell Biology, P.S. Michael, ed. (Academic Press), pp. 157-171.

Dai, J., and Sheetz, M.P. (1999). Membrane tether formation from blebbing cells. Biophysical journal 77, 3363-3370.

Dai, J., Sheetz, M.P., Wan, X., and Morris, C.E. (1998). Membrane tension in swelling and shrinking molluscan neurons. The Journal of neuroscience : the official journal of the Society for Neuroscience 18, 6681-6692.

de Gennes, P.G. (1985). Wetting: statics and dynamics. Reviews of Modern Physics 53, 827-863.

Demerens, C., Stankoff, B., Logak, M., Anglade, P., Allinquant, B., Couraud, F., Zalc, B., and Lubetzki, C. (1996). Induction of myelination in the central nervous system by electrical activity. Proceedings of the National Academy of Sciences of the United States of America 93, 9887-9892.

Dey, A., and Szoszkiewicz, R. (2012). Complete noise analysis of a simple force spectroscopy AFM setup and its applications to study nanomechanics of mammalian Notch 1 protein. Nanotechnology 23, 175101.

Diz-Munoz, A., Fletcher, D.A., and Weiner, O.D. (2013). Use the force: membrane tension as an organizer of cell shape and motility. Trends in cell biology 23, 47-53.

Douezan, S., Guevorkian, K., Naouar, R., Dufour, S., Cuvelier, D., and BrochardWyart, F. (2011). Spreading dynamics and wetting transition of cellular aggregates. Proceedings of the National Academy of Sciences of the United States of America 108, 7315-7320.

Dreyer, J.K., Berg-Sorensen, K., and Oddershede, L. (2004). Improved axial position detection in optical tweezers measurements. Applied optics 43, 1991-1995.

Dyer, C.A., and Benjamins, J.A. (1989). Organization of oligodendroglial membrane sheets. I: Association of myelin basic protein and 2',3'-cyclic nucleotide 3'phosphohydrolase with cytoskeleton. Journal of neuroscience research 24, 201-211.

Eghiaian, F., and Schaap, I.A. (2011). Structural and dynamic characterization of biochemical processes by atomic force microscopy. Methods in molecular biology 778 , 71-95.

Emery, B. (2010). Regulation of oligodendrocyte differentiation and myelination. Science 330, 779-782. 
Engler, A.J., Sen, S., Sweeney, H.L., and Discher, D.E. (2006). Matrix elasticity directs stem cell lineage specification. Cell 126, 677-689.

Fancy, S.P., Baranzini, S.E., Zhao, C., Yuk, D.I., Irvine, K.A., Kaing, S., Sanai, N., Franklin, R.J., and Rowitch, D.H. (2009). Dysregulation of the Wnt pathway inhibits timely myelination and remyelination in the mammalian CNS. Genes \& development 23, 1571-1585.

Fernandez-Valle, C., Gorman, D., Gomez, A.M., and Bunge, M.B. (1997). Actin plays a role in both changes in cell shape and gene-expression associated with Schwann cell myelination. The Journal of neuroscience : the official journal of the Society for Neuroscience 17, 241-250.

Figard, L., Xu, H., Garcia, H.G., Golding, I., and Sokac, A.M. (2013). The plasma membrane flattens out to fuel cell-surface growth during Drosophila cellularization. Developmental cell 27, 648-655.

Fitzner, D., and Simons, M. (2010). Chronic progressive multiple sclerosis pathogenesis of neurodegeneration and therapeutic strategies. Current neuropharmacology 8, 305-315.

Flynn, K.C., Hellal, F., Neukirchen, D., Jacob, S., Tahirovic, S., Dupraz, S., Stern, S., Garvalov, B.K., Gurniak, C., Shaw, A.E., et al. (2012). ADF/cofilin-mediated actin retrograde flow directs neurite formation in the developing brain. Neuron 76, 10911107.

Foty, R.A., and Steinberg, M.S. (2004). Cadherin-mediated cell-cell adhesion and tissue segregation in relation to malignancy. The International journal of developmental biology 48, 397-409.

Franze, K. (2013). The mechanical control of nervous system development. Development 140, 3069-3077.

Fu, H., Cai, J., Clevers, H., Fast, E., Gray, S., Greenberg, R., Jain, M.K., Ma, Q., Qiu, M., Rowitch, D.H., et al. (2009). A genome-wide screen for spatially restricted expression patterns identifies transcription factors that regulate glial development. The Journal of neuroscience : the official journal of the Society for Neuroscience 29, 1139911408.

Geiger, B., and Bershadsky, A. (2002). Exploring the neighborhood: adhesion-coupled cell mechanosensors. Cell 110, 139-142.

Georges, P.C., Miller, W.J., Meaney, D.F., Sawyer, E.S., and Janmey, P.A. (2006). Matrices with compliance comparable to that of brain tissue select neuronal over glial growth in mixed cortical cultures. Biophysical journal 90, 3012-3018.

Gittes, F., and Schmidt, C.F. (1997). Chapter 8 Signals and Noise in Micromechanical Measurements. In Methods in Cell Biology, P.S. Michael, ed. (Academic Press), pp. 129-156.

Guck, J., and Chilvers, E.R. (2013). Mechanics meets medicine. Science translational medicine 5, $212 \mathrm{fs} 241$. 
Harburger, D.S., and Calderwood, D.A. (2009). Integrin signalling at a glance. Journal of cell science $122,159-163$.

Harris, A.K. (1976). Is Cell sorting caused by differences in the work of intercellular adhesion? A critique of the Steinberg hypothesis. Journal of theoretical biology 61, 267285.

Hartline, D.K., and Colman, D.R. (2007). Rapid conduction and the evolution of giant axons and myelinated fibers. Current biology : CB 17, R29-35.

Hertz, H. (1881). Uber die Beruehrung elasticher Koerper (On Contact of Elastic Bodies), Vol 1 (Gesammelte Werke (Collected Works)).

Hinterdorfer, P., and Dufrêne, Y.F. (2006). Detection and localization of single molecular recognition events using atomic force microscopy. Nature methods 3, 347355.

Hochmuth, F.M., Shao, J.Y., Dai, J., and Sheetz, M.P. (1996). Deformation and flow of membrane into tethers extracted from neuronal growth cones. Biophysical journal 70 , 358-369.

Hoffman, B.D., and Crocker, J.C. (2009). Cell mechanics: dissecting the physical responses of cells to force. Annual review of biomedical engineering 11, 259-288.

Hoffman, B.D., Massiera, G., Van Citters, K.M., and Crocker, J.C. (2006). The consensus mechanics of cultured mammalian cells. Proceedings of the National Academy of Sciences of the United States of America 103, 10259-10264.

Hofmann, U.G., Rotsch, C., Parak, W.J., and Radmacher, M. (1997). Investigating the cytoskeleton of chicken cardiocytes with the atomic force microscope. Journal of structural biology 119, 84-91.

Hu, K., Ji, L., Applegate, K.T., Danuser, G., and Waterman-Storer, C.M. (2007). Differential transmission of actin motion within focal adhesions. Science 315, 111-115.

Hu, M., Wang, J., Zhao, H., Dong, S., and Cai, J. (2009). Nanostructure and nanomechanics analysis of lymphocyte using AFM: from resting, activated to apoptosis. Journal of biomechanics 42, 1513-1519.

Hu, S., Chen, J., Fabry, B., Numaguchi, Y., Gouldstone, A., Ingber, D.E., Fredberg, J.J., Butler, J.P., and Wang, N. (2003). Intracellular stress tomography reveals stress focusing and structural anisotropy in cytoskeleton of living cells. American journal of physiology Cell physiology 285, C1082-1090.

Huiskes, R., Ruimerman, R., van Lenthe, G.H., and Janssen, J.D. (2000). Effects of mechanical forces on maintenance and adaptation of form in trabecular bone. Nature 405, 704-706. 
Hutter, J.L., and Bechhoefer, J. (1993). Calibration of atomic-force microscope tips.

Review of Scientific Instruments 64, 1868-1873.

Jacobs, C.R., Temiyasathit, S., and Castillo, A.B. (2010). Osteocyte mechanobiology and pericellular mechanics. Annual review of biomedical engineering 12, 369-400.

Jahn, O., Tenzer, S., and Werner, H.B. (2009). Myelin proteomics: molecular anatomy of an insulating sheath. Molecular neurobiology 40, 55-72.

Kapanidis, A.N., and Strick, T. (2009). Biology, one molecule at a time. Trends in biochemical sciences 34, 234-243.

Knobler, R.L., Stempak, J.G., and Laurencin, M. (1976). Nonuniformity of the oligodendroglial ensheathment of axons during myelination in the developing rat central nervous system. A serial section electron microscopical study. Journal of ultrastructure research 55, 417-432.

Krummel, M.F., Friedman, R.S., and Jacobelli, J. (2014). Modes and mechanisms of T cell motility: roles for confinement and Myosin-IIA. Current opinion in cell biology 30, 9-16.

Kuhlmann-Krieg, S., Sommer, I., and Schachner, M. (1988). Ultrastructural features of cultured oligodendrocytes expressing stage-specific cell-surface antigens. Brain research 467, 269-280.

Kuznetsova, T.G., Starodubtseva, M.N., Yegorenkov, N.I., Chizhik, S.A., and Zhdanov, R.I. (2007). Atomic force microscopy probing of cell elasticity. Micron 38, 824-833.

Lammermann, T., Bader, B.L., Monkley, S.J., Worbs, T., Wedlich-Soldner, R., Hirsch, K., Keller, M., Forster, R., Critchley, D.R., Fassler, R., et al. (2008). Rapid leukocyte migration by integrin-independent flowing and squeezing. Nature 453, 51-55.

Lappe-Siefke, C., Goebbels, S., Gravel, M., Nicksch, E., Lee, J., Braun, P.E., Griffiths, I.R., and Nave, K.A. (2003). Disruption of Cnp1 uncouples oligodendroglial functions in axonal support and myelination. Nature genetics 33, 366-374.

Last, J.A., Russell, P., Nealey, P.F., and Murphy, C.J. (2010). The applications of atomic force microscopy to vision science. Investigative ophthalmology \& visual science 51,6083-6094.

Lee, D.A., Knight, M.M., Campbell, J.J., and Bader, D.L. (2011). Stem cell mechanobiology. Journal of cellular biochemistry 112, 1-9.

Leone, D.P., Genoud, S., Atanasoski, S., Grausenburger, R., Berger, P., Metzger, D., Macklin, W.B., Chambon, P., and Suter, U. (2003). Tamoxifen-inducible glia-specific Cre mice for somatic mutagenesis in oligodendrocytes and Schwann cells. Molecular and cellular neurosciences 22, 430-440. 
Levine, J.M., Reynolds, R., and Fawcett, J.W. (2001). The oligodendrocyte precursor cell in health and disease. Trends in neurosciences 24, 39-47.

Liu, Y.J., Le Berre, M., Lautenschlaeger, F., Maiuri, P., Callan-Jones, A., Heuze, M., Takaki, T., Voituriez, R., and Piel, M. (2015). Confinement and low adhesion induce fast amoeboid migration of slow mesenchymal cells. Cell 160, 659-672.

Lu, Y.B., Franze, K., Seifert, G., Steinhauser, C., Kirchhoff, F., Wolburg, H., Guck, J., Janmey, P., Wei, E.Q., Kas, J., et al. (2006). Viscoelastic properties of individual glial cells and neurons in the CNS. Proceedings of the National Academy of Sciences of the United States of America 103, 17759-17764.

Maier, O., Hoekstra, D., and Baron, W. (2008). Polarity development in oligodendrocytes: sorting and trafficking of myelin components. Journal of molecular neuroscience : MN 35, 35-53.

Maitre, J.L., Berthoumieux, H., Krens, S.F., Salbreux, G., Julicher, F., Paluch, E., and Heisenberg, C.P. (2012). Adhesion functions in cell sorting by mechanically coupling the cortices of adhering cells. Science 338, 253-256.

Martin, Y., Williams, C.C., and Wickramasinghe, H.K. (1987). Atomic force

microscope-force mapping and profiling on a sub 100- $\AA$ scale. Journal of Applied

Physics 61, 4723-4729.

Maxwell, J.C. (1866). On the viscosity or internal friction of air and other gases. Philosophical Transactions of the Royal Society of London 156, 249-268.

Miller, R.H. (2002). Regulation of oligodendrocyte development in the vertebrate CNS. Progress in neurobiology 67, 451-467.

Milner, R., Frost, E., Nishimura, S., Delcommenne, M., Streuli, C., Pytela, R., and Ffrench-Constant, C. (1997). Expression of alpha vbeta3 and alpha vbeta8 integrins during oligodendrocyte precursor differentiation in the presence and absence of axons. Glia 21, 350-360.

Mitchison, T., and Kirschner, M. (1988). Cytoskeletal dynamics and nerve growth. Neuron 1, 761-772.

Moffitt, J.R., Chemla, Y.R., Smith, S.B., and Bustamante, C. (2008). Recent advances in optical tweezers. Annual review of biochemistry 77, 205-228.

Moyer, R.F., Ratneswaran, A., Beier, F., and Birmingham, T.B. (2014). Osteoarthritis year in review 2014: mechanics--basic and clinical studies in osteoarthritis. Osteoarthritis and cartilage / OARS, Osteoarthritis Research Society 22, 1989-2002.

Muller, D.J. (2008). AFM: a nanotool in membrane biology. Biochemistry 47, 79867998. 
Nave, K.A. (2010). Myelination and support of axonal integrity by glia. Nature 468 , 244-252.

Nawaz, S., Sanchez, P., Bodensiek, K., Li, S., Simons, M., and Schaap, I.A. (2012). Cell visco-elasticity measured with AFM and optical trapping at sub-micrometer deformations. PloS one 7, e45297.

Neuman, K.C., and Nagy, A. (2008). Single-molecule force spectroscopy: optical tweezers, magnetic tweezers and atomic force microscopy. Nature methods 5, 491-505.

Neuroscience (2004). PURVES, D., AUGUSTINE, G., FITZPATRICK, D., et al.; editors, 3 edn (Sinauer Associates).

Pelling, A.E., and Horton, M.A. (2008). An historical perspective on cell mechanics. Pflugers Archiv : European journal of physiology 456, 3-12.

Pelling, A.E., Veraitch, F.S., Chu, C.P., Mason, C., and Horton, M.A. (2009). Mechanical dynamics of single cells during early apoptosis. Cell motility and the cytoskeleton $66,409-422$.

Peterman, E.J., Gittes, F., and Schmidt, C.F. (2003). Laser-induced heating in optical traps. Biophysical journal 84, 1308-1316.

Putman, C.A.J., Van der Werf, K.O., De Grooth, B.G., Van Hulst, N.F., and Greve, J. (1994). Tapping mode atomic force microscopy in liquid. Applied Physics Letters 64, 2454-2456.

Raff, M.C., Miller, R.H., and Noble, M. (1983). A glial progenitor cell that develops in vitro into an astrocyte or an oligodendrocyte depending on culture medium. Nature 303, 390-396.

Raucher, D., and Sheetz, M.P. (1999). Characteristics of a membrane reservoir buffering membrane tension. Biophysical journal 77, 1992-2002.

Renkawitz, J., Schumann, K., Weber, M., Lammermann, T., Pflicke, H., Piel, M., Polleux, J., Spatz, J.P., and Sixt, M. (2009). Adaptive force transmission in amoeboid cell migration. Nature cell biology 11, 1438-1443.

Renkawitz, J., and Sixt, M. (2010). Mechanisms of force generation and force transmission during interstitial leukocyte migration. EMBO reports 11, 744-750.

Ridley, A.J. (2011). Life at the leading edge. Cell 145, 1012-1022.

Rogister, B., Ben-Hur, T., and Dubois-Dalcq, M. (1999). From neural stem cells to myelinating oligodendrocytes. Molecular and cellular neurosciences 14, 287-300.

Rotsch, C., and Radmacher, M. (2000). Drug-induced changes of cytoskeletal structure and mechanics in fibroblasts: an atomic force microscopy study. Biophysical journal 78 , 520-535. 
Ruprecht, V., Wieser, S., Callan-Jones, A., Smutny, M., Morita, H., Sako, K., Barone, V., Ritsch-Marte, M., Sixt, M., Voituriez, R., et al. (2015). Cortical contractility triggers a stochastic switch to fast amoeboid cell motility. Cell 160, 673-685.

Sachs, F. (2010). Stretch-activated ion channels: what are they? Physiology 25, 50-56.

Sackmann, E., and Bruinsma, R.F. (2002). Cell adhesion as wetting transition? Chemphyschem : a European journal of chemical physics and physical chemistry 3, 262-269.

Sadd, M.H. (2005). Elasticity: Theory, Applications and Numerics (Elsevier Butterworth-Heinemann).

Schmitt, S., Cantuti Castelvetri, L., and Simons, M. (2014). Metabolism and functions of lipids in myelin. Biochimica et biophysica acta.

Sheetz, M.P. (2001). Cell control by membrane-cytoskeleton adhesion. Nature reviews Molecular cell biology 2, 392-396.

Sheetz, M.P., and Dai, J. (1996). Modulation of membrane dynamics and cell motility by membrane tension. Trends in cell biology $6,85-89$.

Simons, M., and Trajkovic, K. (2006). Neuron-glia communication in the control of oligodendrocyte function and myelin biogenesis. Journal of cell science 119, 43814389.

Snaidero, N., Mobius, W., Czopka, T., Hekking, L.H., Mathisen, C., Verkleij, D., Goebbels, S., Edgar, J., Merkler, D., Lyons, D.A., et al. (2014). Myelin membrane wrapping of CNS axons by $\mathrm{PI}(3,4,5) \mathrm{P} 3$-dependent polarized growth at the inner tongue. Cell 156, 277-290.

Sobottka, B., Ziegler, U., Kaech, A., Becher, B., and Goebels, N. (2011). CNS live imaging reveals a new mechanism of myelination: the liquid croissant model. Glia 59, 1841-1849.

Stevenson, D.J., Gunn-Moore, F., and Dholakia, K. (2010). Light forces the pace: optical manipulation for biophotonics. Journal of biomedical optics 15, 041503.

Svitkina, T.M. (2013). Ultrastructure of protrusive actin filament arrays. Current opinion in cell biology 25, 574-581.

Tiwari-Woodruff, S.K., Buznikov, A.G., Vu, T.Q., Micevych, P.E., Chen, K., Kornblum, H.I., and Bronstein, J.M. (2001). OSP/claudin-11 forms a complex with a novel member of the tetraspanin super family and betal integrin and regulates proliferation and migration of oligodendrocytes. The Journal of cell biology 153, 295305.

Trapp, B.D., Andrews, S.B., Wong, A., O'Connell, M., and Griffin, J.W. (1989). Colocalization of the myelin-associated glycoprotein and the microfilament components, F-actin and spectrin, in Schwann cells of myelinated nerve fibres. Journal of neurocytology $18,47-60$. 
Trotter, J., and Schachner, M. (1989). Cells positive for the O4 surface antigen isolated by cell sorting are able to differentiate into astrocytes or oligodendrocytes. Brain research Developmental brain research 46, 115-122.

Vermeulen, K.C., Wuite, G.J., Stienen, G.J., and Schmidt, C.F. (2006). Optical trap stiffness in the presence and absence of spherical aberrations. Applied optics 45, 18121819.

Verschueren, H. (1985). Interference reflection microscopy in cell biology: methodology and applications. Journal of cell science 75, 279-301.

Vinckier, A., and Semenza, G. (1998). Measuring elasticity of biological materials by atomic force microscopy. FEBS letters 430, 12-16.

Viswanath, D.S., Ghosh, T.K., Prasad, D.H.L., Dutt, N.V.K., and Rani, K.Y. (2007). Viscosity of Liquids: Theory, Estimation, Experiment, and Data (Springer).

Vouyiouklis, D.A., and Brophy, P.J. (1997). A novel gelsolin isoform expressed by oligodendrocytes in the central nervous system. Journal of neurochemistry 69, 9951005 .

Webster, H.D. (1971). The geometry of peripheral myelin sheaths during their formation and growth in rat sciatic nerves. The Journal of cell biology 48, 348-367.

Zhang, H., and Liu, K.K. (2008). Optical tweezers for single cells. Journal of the Royal Society, Interface / the Royal Society 5, 671-690.

Zuchero, J.B., and Barres, B.A. (2011). Between the sheets: a molecular sieve makes myelin membranes. Developmental cell 21, 385-386. 


\section{Curriculum Vitae}

\section{Paula Verónica Sánchez Baeza.}

\begin{tabular}{ll} 
Personal data & \\
\hline & \\
Date of birth & $27 / 09 / 1985$ \\
Place of birth & Temuco, Chile \\
Nationality & Chilean \\
Address & Max Planck Institute for Experimental Medicine \\
& Hermann-Rein_Str. 3 \\
& 37075, Göttingen \\
& Germany \\
Phone number & (+49) 551 3899532 \\
Email & psanchez@em.mpg.de
\end{tabular}

\section{Education}

May 2011- Current

$\mathrm{PhD}$ student Georg-August-University, Göttingen, Germany. Proyect "Actin turnover regulates mechanical properties of oligodendrocytes and myelin formation". Supervisor Iwan A. T. Schaap.

\section{August 2008- April 2011}

Diploma thesis in Cell Biology. Pontificia Universidad Católica de Chile.

\section{March 2004- August 2008}

Bachelor degree in Biochemistry. Pontificia Universidad Católica de Chile.

\section{Publications}

Nawaz S.*, Sánchez P.*, Schmitt S., Snaidero N., Mitkovski M., Velte C., Brückner B.R., Alexopoulos I., Czopka T., Jung S.Y. ,Rhee J.S., Janshoff A., Witke W., Schaap I.A., Lyons D.A., Simons M. (2015). Actin filament turnover drives leading edge growth during myelin sheath formation in the central nervous system. Developmental cell, accepted.

* Both authors contributed equally to this work

Otero C., Linke M., Sánchez P., González A., Schaap I.A. (2013). Propranolol restricts the mobility of single EGF-receptors on the cell surface before their internalization. PloS one 8(12): e83086

Bodensiek, K., Li, W., Sánchez, P., Nawaz, S., and Schaap, I.A. (2013). A high-speed vertical optical trap for the mechanical testing of living cells at piconewton forces. The Review of scientific instruments 84(11):113707 
Aggarwal S., Snaidero N., Pähler G., Frey S., Sánchez P., Zweckstetter M., Janshoff A., Schneider A., Weil M., Schaap I.A.T., Görlich D., Simons M. (2013) Myelin membrane assembly is driven by phase transition of myelin basic protein into a cohesive meshwork. PLoS Biology 11(6):e1001577

Nawaz S.*, Sánchez P.*, Bodensiek K., Li S., Simons M., Schaap I.A.T. (2012) Cell Visco-Elasticity Measured with AFM and Optical Trapping at Sub-Micrometer Deformations. PloS one 7(9): e45297

* Both authors contributed equally to this work

\section{Oral Presentations at meetings}

PDE4D Regulates Ligand-Independent EGFR Endocytosis

Sánchez P, Otero C, González A.

XXIV Annual Meeting of the Chilean Society for Cell Biology. November, 2010 Pucón, Chile.

\section{Poster Presentations at meetings}

Mechanics of myelin sheath formation

Sanchez P, Nawaz S, Simons M, Schaap I. A. T.

Mechanics of cells. September, 2013. Obergurgl, Austria.

Cell visco-elasticity measured with afm and optical trapping at sub-micrometer deformations

Sanchez P, Li W, Zeiss M, Wollnik C, Bodensiek K, Rehfeldt F, Schaap I. A. T.

DPG-Spring meeting. March, 2013. Regensburg, Germany.

Cell visco-elasticity measured with afm and optical trapping at sub-micrometer deformations

Sanchez P, Nawaz S, Bodensiek K, Li S, Simons M, Schaap I. A. T.

Physics of cells. September, 2012. Hyères, France.

Measuring local elasticity and membrane tension on differentiating cells

Sanchez P, Bodensiek K, Nawaz S, Simons M, Schaap I. A. T.

DPG-Spring meeting. March, 2012. Berlin, Germany.

\section{Teaching experience}

April- July 2013

May- July 2012

March- July 2010

March- July 2009

August- December 2008
Teaching Assistant. Georg-August-University. Course: Master Forschungspraktikum: Stiffness measurements on biological materials using atomic force microscopy

Teaching Assistant. Georg-August-University. Course: Master Forschungspraktikum: Stiffness measurements on biological materials using atomic force microscopy

Teaching Assistant. Pontificia Universidad Católica de Chile. Course: Molecular Biology of the Cell (Bio 238)

Teaching Assistant. Pontificia Universidad Católica de Chile. Course: Physical Bases of Biological Processes (Bio 152)

Teaching Assistant. Pontificia Universidad Católica de Chile. Course: Laboratory of Biochemistry II: Molecular Genetics (Bio 266) 


\section{List of publications}

Nawaz S.*, Sánchez P.*, Schmitt S., Snaidero N., Mitkovski M., Velte C., Brückner B.R., Alexopoulos I., Czopka T., Jung S.Y. ,Rhee J.S., Janshoff A., Witke W., Schaap I.A., Lyons D.A., Simons M. (2015). Actin filament turnover drives leading edge growth during myelin sheath formation in the central nervous system. Developmental cell, accepted.

* Equal contribution

Otero C., Linke M., Sánchez P., González A., Schaap I.A. (2013). Propranolol restricts the mobility of single EGF-receptors on the cell surface before their internalization. PloS one 8(12): e83086

Bodensiek, K., Li, W., Sánchez, P., Nawaz, S., and Schaap, I.A. (2013). A high-speed vertical optical trap for the mechanical testing of living cells at piconewton forces. The Review of scientific instruments 84(11):113707

Aggarwal S., Snaidero N., Pähler G., Frey S., Andreas Janshoff A., Sánchez P., MarieTheres Weil M.T., Schaap I.A., Görlich D., Simons M. (2013). Myelin membrane assembly is driven by a phase transition of myelin basic proteins into a cohesive protein meshwork. PloS Biology 11(6):e1001577

Nawaz S*., Sánchez P*., Bodensiek K., Li S., Simons M., Schaap I.A. (2012). Cell visco-elasticity measured with AFM and optical trapping at sub-micrometer deformations. PloS one 7(9):e45297

* Equal contribution 